\title{
High-Fidelity Qubit Readout Using Interferometric Directional Josephson Devices
}

\author{
Baleegh Abdo $\odot,{ }^{*}$ Oblesh Jinka $\odot$, Nicholas T. Bronn๑, Salvatore Olivadese $\odot$, and Markus Brink \\ IBM Quantum, IBM Research Center, Yorktown Heights, New York 10598, USA
}

(Received 4 February 2021; revised 29 September 2021; accepted 24 November 2021; published 28 December 2021)

\begin{abstract}
Nonreciprocal microwave devices, such as circulators and isolators, are needed in high-fidelity qubit readout schemes to unidirectionally route the readout signals and protect the qubits against noise coming from the output chain. However, cryogenic circulators and isolators are prohibitive in scalable superconducting architectures because they rely on magnetic materials. Here we report a fast (750 ns) high-fidelity (95\%) quantum nondemolition readout of a coherent superconducting qubit $\left(T_{1}=52 \mu \mathrm{s}, T_{2 E}=35 \mu \mathrm{s}\right)$ without any nonreciprocal magnetic devices. We use in our readout chain a microwave-controlled qubit readout multichip module (QRMCM) that integrates interferometric directional Josephson devices consisting of an isolator and a reconfigurable isolator or amplifier device, and an off-chip low-pass filter. Using the QRMCM, we demonstrate isolation up to $45 \mathrm{~dB}$ within $13 \mathrm{MHz}$, when both directional devices are operated as isolators, and low-noise amplification in excess of $10 \mathrm{~dB}$ within a dynamical bandwidth of $10 \mathrm{MHz}$, when the reconfigurable device is operated as an amplifier. We also investigate the dependence of the qubit coherence times $T_{\varphi}$ and $T_{2 E}$ on the isolation response of the QRMCM, which we control in situ using the microwave drives feeding the isolators. Furthermore, by directly comparing the QRMCM performance with that of a state-of-art configuration (with $T_{2 E} \approx 2 T_{1}$ ) that uses a pair of wideband magnetic isolators, we find that the excess pure dephasing measured with the QRMCM (for which $T_{2 E} \approx T_{1}$ ) is likely limited by the residual thermal photon population in the readout resonator. Improved versions of the QRMCM could replace magnetic circulators and isolators in large superconducting quantum processors.
\end{abstract}

DOI: 10.1103/PRXQuantum.2.040360

\section{INTRODUCTION}

Nonreciprocity breaks the transmission-coefficient symmetry for light upon exchange of sources and detectors. A common method for breaking reciprocity is guiding light through magnetic materials exhibiting magneto-optical effects, such as the Faraday effect $[1,2]$. Other nonreciprocity schemes include operating in the nonlinear regime [3], using the quantum Hall effect [4-6], and parametrically modulating a certain physical property of the system [7-12].

Because of their ability to break the transmission symmetry, nonreciprcocal devices play critical roles in a variety of basic science and technology applications, requiring, for example, signal transport control, separation of input from output in reflective or communication setups, and source protection against backscatter or detector backaction. In particular, in the realm of superconducting

\footnotetext{
*babdo@us.ibm.com

Published by the American Physical Society under the terms of the Creative Commons Attribution 4.0 International license. Further distribution of this work must maintain attribution to the author(s) and the published article's title, journal citation, and DOI.
}

quantum processors, nonreciprocal microwave devices, such as circulators and isolators, are critical for performing high-fidelity quantum nondemolition measurements [1318]. With relatively low loss, they route readout signals to and from quantum processors in a directional manner and protect qubits against noise coming from the output chain. However, state-of-the-art cryogenic circulators and isolators are prohibitive in scalable architectures because they are bulky and rely on magnetic materials and strong magnetic fields $[19,20]$, which are difficult to integrate on a chip and are incompatible with superconducting circuits.

In an attempt to solve this scalability challenge, a variety of viable alternative circulator and isolator schemes have been proposed and realized, which use photonic transitions between coupled resonance modes [21,22], the Hall effect [6], frequency conversion in nonlinear transmission lines [23], frequency conversion combined with delay lines [24,25], dynamical modulation of transfer switches incorporated with delay lines [26], and reservoir engineered optomechanical interactions [27-29]. However, despite this great progress in the development of alternative directional devices and readout schemes, demonstrating high-fidelity dispersive readout of a superconducting qubit with relatively high coherence 
without using any magnetic isolators and circulators in the readout chain remains an outstanding challenge [30-33]. Here we achieve this milestone by building a qubit readout multichip module (QRMCM) that integrates into a printed circuit board (PCB) interferometric directional Josephson devices, a superconducting directional coupler, and a Purcell filter and by incorporating an off-the-shelf low-pass microwave filter at the output of the QRMCM. Also, importantly, our readout scheme operates in continuous-wave mode, which is highly beneficial in scalable architectures because it is compatible with frequency-multiplexed readout, unlike, for example, a superconducting low-inductance undulatory galvanometer microwave amplifier, which exhibits a strong inherent reverse isolation but operates in pulsed mode [34].

Furthermore, owing to the concatenation of more than one directional device in the QRMCM, we achieve an isolation record in a nonreciprocal superconducting circuit of about $45 \mathrm{~dB}$ at the readout frequency. Moreover, by varying the isolation in situ, enabled by the QRMCM, we investigate the dependence of qubit dephasing on the isolator's performance and noise originating from the 4$\mathrm{K}$ stage and the high-electron-mobility-transistor (HEMT) amplifier (which is a long-standing, critical microwave component in superconducting qubit readout chains).

Central to achieving these results are the multipath interoferometric Josephson isolator (MPIJIS) [35] and a reconfigurable directional device that can be operated as a MPIJIS or a multipath interoferometric Josephson directional amplifier (MPIJDA) [36,37], both of which are key components in the proof-of-principle QRMCM presented here. The MPIJIS is formed by our coupling two nominally identical nondegenerate Josephson mixers [38] via their respective distinct spatial and spectral eigenmodes, a and b. One mode in this scheme (e.g., mode a) supports input and output propagating signals within the device bandwidth, while the other (e.g., mode b) serves as an internal mode of the system coupled to a dissipation port. By parametrically modulating the inductive coupling between the modes, we generate an artificial gauge-invariant potential for microwave photons, which imprints nonreciprocal phase shifts on the transmitted signals through the mixers, undergoing frequency conversion. By further embedding the mixers in an interferometric setup, we create a unidirectional transmission of propagating signals owing to constructive and destructive wave interference occurring between different paths in the device.

However, unlike the MPIJIS device presented in Ref. [35], which (1) integrates on-chip superconducting and off-chip normal-metal circuits and (2) requires, for its operation, two same-frequency phase-locked microwave drives injected through two input lines in the dilution fridge, the present MPIJIS is a superconducting on-chip device that is operated by a single microwave drive fed through one input line. These changes lead to several key advantages, such as (1) eliminating the losses in the normal metal parts, (2) paving the way for size reduction using lumpedelement designs, and (3) requiring only one microwave source and input line, on a par with Josephson parametric amplifiers (JPAs). The latter leads to savings in the hardware resources required per qubit, easier tune-up procedures, enhanced stability over time, and simpler control circuitry. These gains become even more pronounced when we compare this single-pump device with nonreciprocal schemes, which require multiple pumps [11,21,22].

Similarly, the MPIJDA device, which is the dual of the MPIJIS device, shares the same circuit as the MPIJIS but operates in a different mode. More specifically, the nondegenerate Josephson mixers of the device are operated in low-gain amplification mode instead of frequency conversion (without photon gain) mode, which is primarily set by the pump frequency driving the mixers (i.e., the sum of the resonance frequencies of modes $a$ and $b$ in the MPIJDA case and their difference in the MPIJIS case).

The outline of the remainder of the paper is as follows. In Sec. II, we present the MPIJIS device. In Sec. III, we present scattering-parameter measurements of a singlepump MPIJIS. In Sec. IV, we introduce the QRMCM and the qubit setup used for characterization. In Sec. IV A, we report a high-fidelity qubit readout using the QRMCM by our utilizing one MPIJIS and operating the reconfigurable directional device as an amplifier. In Sec. IV B, we report measurement of the qubit while operating the reconfigurable directional device as a second isolator in series. In Sec. IV C, we investigate the dependence of the qubit coherence times on the variable isolation response of the QRMCM. In Sec. IV D, we perform a direct comparison of the qubit coherence times obtained with the QRMCM and two cryogenic magnetic isolators. In Sec. V, we discuss the measurement results and outline possible enhancements to the MPIJIS, MPIJDA, and QRMCM. Finally, in Sec. VI, we conclude by providing a summary of the results and a brief outlook.

\section{THE MPIJIS DEVICE}

Isolators, whose circuit symbol is shown in Fig. 1(a), are two-port microwave devices, which transmit microwave signals propagating in the direction of the arrow (i.e., from port 1 to port 2) at frequency $f_{1}$ (within the device bandwidth), while blocking signals propagating in the opposite direction. Here we realize an on-chip superconducting MPIJIS that requires a single pump for its operation, as illustrated in Figs. 1(b) and 1(c). The basic building block of the device, photographs of which are shown in Figs. 1(e) and 1(f), is the Josephson parametric converter (JPC) [38-41], which functions as a lossless nondegenerate three-wave mixing device. The JPC, as illustrated in Fig. 1(g), comprises two half-wavelength, microstrip resonators denoted "a" and "b," which intersect in the 
(a)
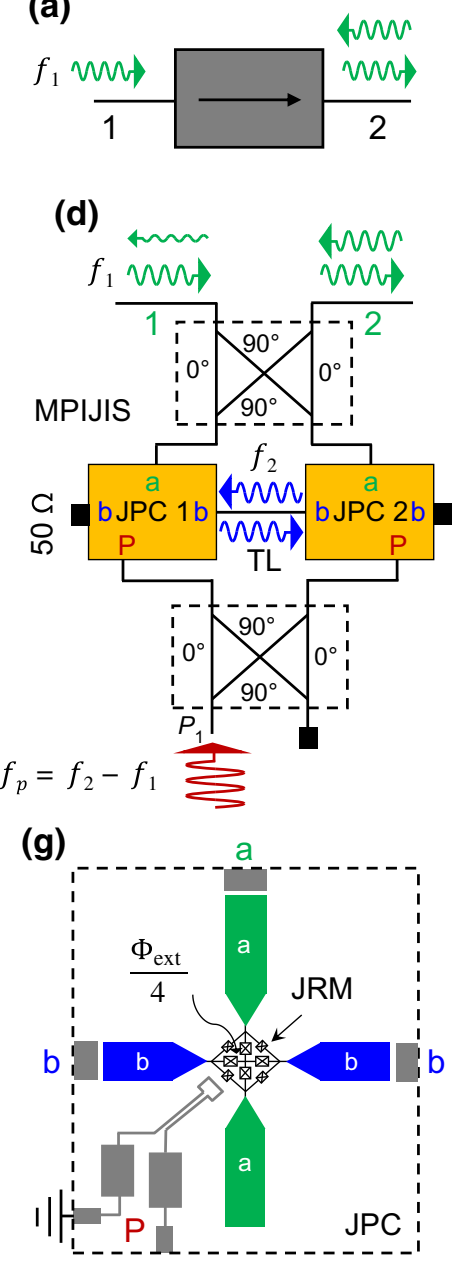

(b)

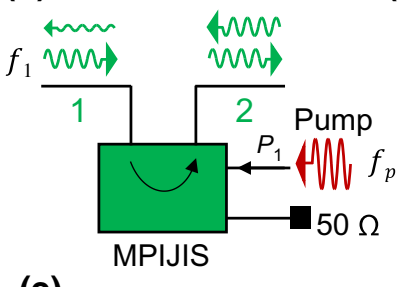

(e)

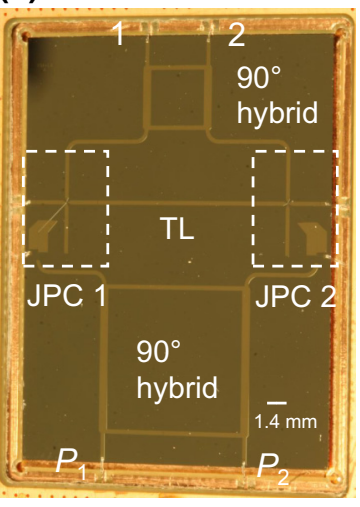

(f)
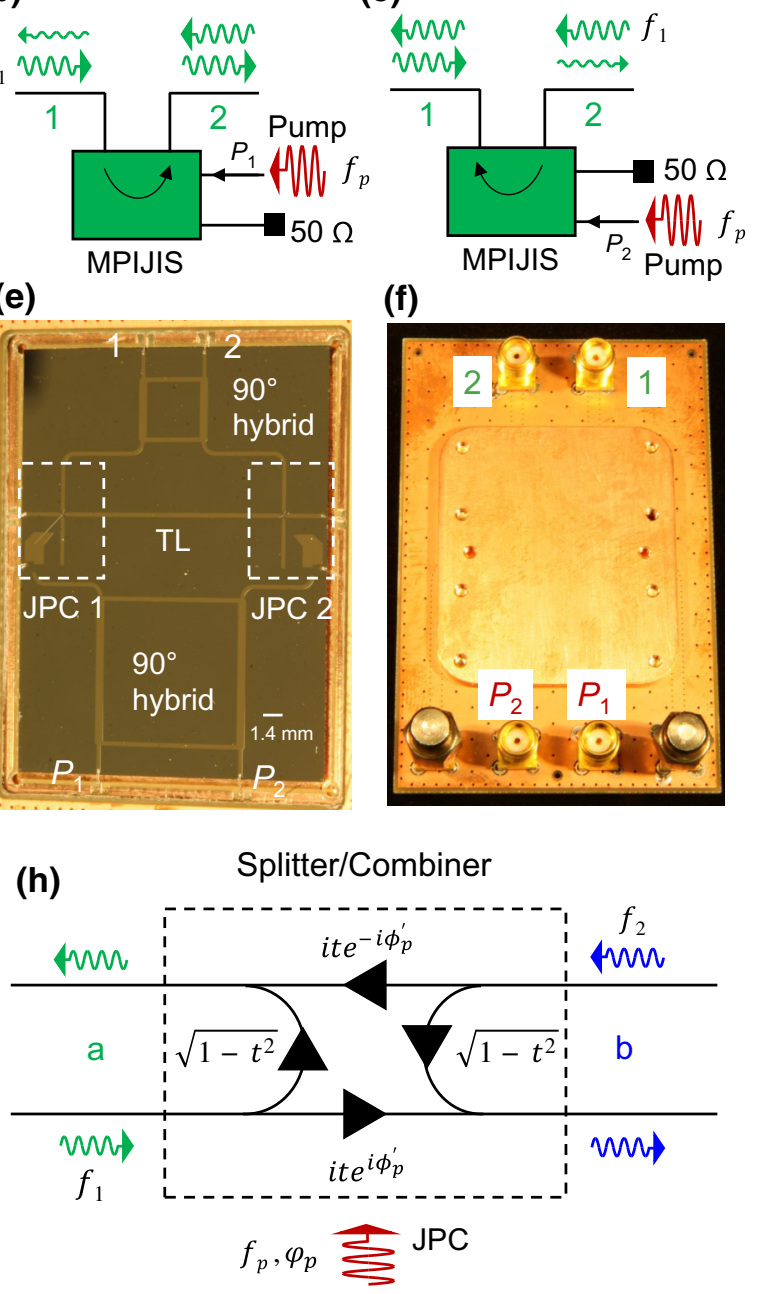

FIG. 1. On-chip single-pump MPIJIS. (a) Isolator circuit symbol. In ideal isolators, input signals propagating in the direction of the arrow are fully transmitted; signals propagating in the opposite direction are blocked. (b),(c) Circuit symbols for the MPIJIS. The direction of the arrow depends on which pump port of the device is driven. (d) Block diagram of the MPIJIS scheme. It consists of two identical JPCs coupled in an interferometric setup. (e) Photograph of the MPIJIS chip. The two JPCs, the signal hybrid, the coupling transmission line (TL), and the pump hybrid are all superconducting and are realized on the same chip. (f) Photograph of the PCB and bottom cover of the MPIJIS. The length and width of the PCB are $7.62 \mathrm{~cm}$ and $5.08 \mathrm{~cm}$, respectively. (g) JPC design used in the MPIJIS. (h) Signal flow graph of the JPC operated in frequency-conversion mode without photon gain Signals impinging on ports a and $\mathrm{b}$ undergo frequency conversion and transmission to the other port with amplitude $t$ and are reflected with amplitude $\sqrt{1-t^{2}}$. The transmitted signal acquires a nonreciprocal phase shift $\pm \phi_{p}^{\prime}$, where $\phi_{p}^{\prime}$ is the generalized pump phase.

middle at an inductively shunted Josephson ring modulator (JRM) [42], serving as a dispersive nonlinear medium. The resonators are characterized by flux-tunable resonance frequencies $\omega_{a}\left(\Phi_{\text {ext }}\right) / 2 \pi$ and $\omega_{b}\left(\Phi_{\text {ext }}\right) / 2 \pi$, where $\Phi_{\text {ext }}$ is the external magnetic flux threading the JRM loop, and bandwidths $\gamma_{a} / 2 \pi$ and $\gamma_{b} / 2 \pi$ set by the capacitive coupling to the external feedlines. As illustrated in Fig. 1(g), resonator $\mathrm{a}$ is open-ended and has one feedline, resonator $\mathrm{b}$ has one feedline on each side, and the pump drive is fed to the device via a separate on-chip feedline (marked "P"). The three-wave mixing operation of the JPC is captured by the leading nonlinear term in the system Hamiltonian given by $\mathcal{H}_{3 \text { wave }}=\hbar g_{3}\left(a+a^{\dagger}\right)\left(b+b^{\dagger}\right)\left(c+c^{\dagger}\right)$ [38], where $g_{3}$ is a flux-dependent coupling strength, $a$ and $b$ are the annihilation operators for the differential modes $a$ and $b$, and $c$ is the annihilation operator for the common mode $c$. When a strong, coherent, off-resonant, common drive is applied at $\omega_{p}=\omega_{b}+\omega_{a}$, the JPC functions as a nondegenerate quantum-limited amplifier [38,41], whereas when pumping is applied at $\omega_{p}=\left|\omega_{b}-\omega_{a}\right|$, it behaves as a lossless frequency converter between mode $a$ and mode $b$ $[38,43,44]$. Under the latter classical drive corresponding to the frequency difference, we obtain in the rotatingwave approximation $\mathcal{H}_{3 \text { wave }}=\hbar\left|g_{a b}\right|\left(e^{i \phi_{p}^{\prime}} a b^{\dagger}+e^{-i \phi_{p}^{\prime}} a^{\dagger} b\right)$, 
where $g_{a b}$ is a pump-amplitude-dependent coupling strength (proportional to $g_{3}$ ) and $\phi_{p}^{\prime}$ is a generalized pump phase, which is related to the applied pump phase $\phi_{p}$ by $\phi_{p}^{\prime}=\phi_{p}+n_{g} \pi$, where

$$
n_{g}= \begin{cases}0, & \varphi_{\mathrm{ext}} \leq 0, \\ 1, & \varphi_{\mathrm{ext}}>0 .\end{cases}
$$

The phase added to $\phi_{p}$, i.e., $0\left(n_{g}=0\right)$ or $\pi\left(n_{g}=1\right)$, depends on whether $g_{3}$ is positive or negative, respectively, which is determined by the sign of $\varphi_{\text {ext }}=2 \pi \Phi_{\text {ext }} / \Phi_{0}$, where $\Phi_{0}=h / 2 e$ is the flux quantum, or alternatively the sign of the direct current circulating in the JRM loop. Equation (1) assumes that the JPCs are flux-biased at the primary flux lobe centered at zero flux.

On resonance, the transmission amplitude associated with the frequency-conversion process is given by $t=$ $2 \rho /\left(1+\rho^{2}\right)$, where $\rho=2\left|g_{a b}\right| / \sqrt{\gamma_{a} \gamma_{b}}$ is a dimensionless pump amplitude, which ranges between 0 (total reflection) and 1 (full conversion). Notably, increasing $\rho$ beyond 1 is possible in conversion mode but it results in lower $t$ than its maximum at $\rho=1$.

As seen in the block diagram in Fig. 1(d), the MPIJIS is formed by our coupling two nominally identical JPCs in an interferometric setup, where mode a of the JPCs is coupled via a $90^{\circ}$ hybrid and mode $b$ is coupled to external $50-\Omega$ cold terminations and an intermediate transmission line. The device also includes a $90^{\circ}$ hybrid for the pump that is connected to the pump feedlines of the JPCs. Since this hybrid splits the pump evenly between the two stages and imposes, by design, the required phase difference for nonreciprocity (i.e., $\pm \pi / 2$ ), the device operates using a single drive.

Assuming symmetric coupling between modes b of the JPCs and the cold terminations, the transmission parameters of the MPIJIS on resonance are given by (see Appendix A)

$$
S_{2 \leftrightarrows 1}=i \frac{\sqrt{1-t^{2}} \mp \sqrt{2} t^{2} \sin \varphi}{1+t^{2}},
$$

and the reflection parameters read

$$
S_{11}=S_{22}=-i \frac{\sqrt{2} t^{2} \cos \varphi}{1+t^{2}},
$$

where $\varphi \equiv \varphi_{p}+p \pi, \varphi_{p} \equiv \phi_{p 1}-\phi_{p 2}$ is the phase difference between the same-frequency pumps feeding the two stages, and $p \equiv\left(n_{g 1}+n_{g 2}\right)$ mod 2 is the parity of the applied fluxes threading the two JRMs.

From these definitions and Eq. (1), it follows that (1) $\varphi_{p}=\mp \pi / 2$ when ports $P_{1}$ and $P_{2}$ are driven, respectively, (2) $p=0$ when the signs of $\varphi_{\mathrm{ext1}}$ and $\varphi_{\mathrm{ext} 2}$ are the same (i.e., even parity), and (3) $p=1$ when their signs are opposite (i.e., odd parity); for further details see Appendixes A, B, F, and G.

By operating the two JPCs in frequency-conversion mode at the 50:50 beam splitting point (i.e., $t=1 / \sqrt{2}$ ), in which half of the signal input on each port is reflected and half is transmitted with frequency conversion to the other port [as illustrated in the signal flow graph in Fig. 1(h)], and by setting, for example, the generalized phase difference to $\varphi=\varphi_{p}=-\pi / 2$, attained in the case of pumping through $P_{1}$ while $p=0$, we generate a nonreciprocal response, in which input signals on resonance propagating in the direction of the arrow shown in Fig. 1(b) are transmitted with near-unity transmission, i.e., $\left|S_{21}\right|=2 \sqrt{2} / 3 \simeq$ 0.94 (equivalent to a loss of about $0.5 \mathrm{~dB}$ in signal power), whereas signals propagating in the opposite direction are blocked, i.e., $S_{12}=0$ (i.e., routed to the cold terminations), and reflections vanish, i.e., $S_{11}=S_{22}=0$.

\section{MPIJIS MEASUREMENTS}

\section{A. Scattering parameters}

Figure 2 shows a measurement of the scattering parameters of the MPIJIS operated in two modes of operation at fixed applied fluxes, for which $p=0$. In the first, the MPIJIS is driven through $P_{1}$, setting its directionality from port 1 to port 2, while in the second, it is driven through $P_{2}$, which reverses its directionality as illustrated in the block diagrams in the left column. The input power applied in the scattering parameter measurements in this work, unless stated otherwise, is about $-140 \mathrm{dBm}$, which is much lower than the saturation power of the MPIJIS. In Fig. 2(a), we show the transmission parameters $\left|S_{21}\right|^{2}$ (blue) and $\left|S_{12}\right|^{2}$ (orange) corresponding to the first case. The dashed and solid lines correspond to the MPIJIS being off (no pump) and on (with pump), respectively. Similarly, Fig. 2(c) shows the reversed transmission response corresponding to the second case. In both cases, the MPIJIS response yields on resonance, at $f_{a}=\omega_{a} / 2 \pi=6.84 \mathrm{GHz}$, attenuation of about $2 \mathrm{~dB}$ in the forward direction and $23 \mathrm{~dB}$ in the backward (isolated) direction with a dynamical bandwidth of $8 \mathrm{MHz}$. The corresponding $f_{b}=\omega_{b} / 2 \pi=9.598 \mathrm{GHz}$ is given by $f_{a}+f_{p}$, where $f_{p}=2.758 \mathrm{GHz}$ is the applied pump frequency in this measurement. Figures 2(b) and 2(d) depict the reflection parameters $S_{11}$ (red) and $S_{22}$ (magenta) measured for the respective directionality cases. The magnitude of the measured transmission and reflection parameters of the device is calibrated by means of the experimental procedure outlined in Appendix H. As seen in Figs. 2(b) and 2(d) the reflections off the two ports are small when the pump is on and off, depicted as solid and dashed lines, respectively. In addition to demonstrating that the on-chip single-pump MPIJIS works as intended, the results in Fig. 2 directly confirm the theory prediction that the phase gradient condition for nonreciprocity is $\pm \pi / 2$, imposed by the pump hybrid. 


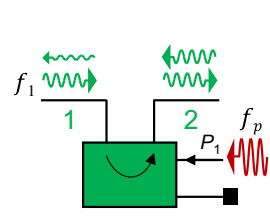

(a) Transmission (dB)
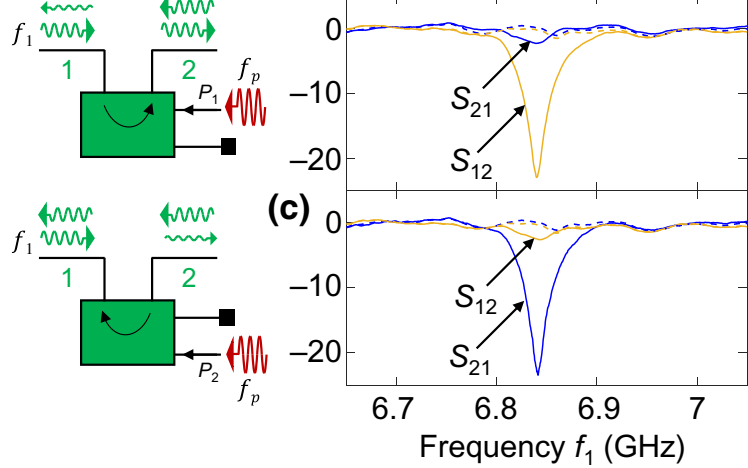

(b)

-10
-20
-30

(d) 0

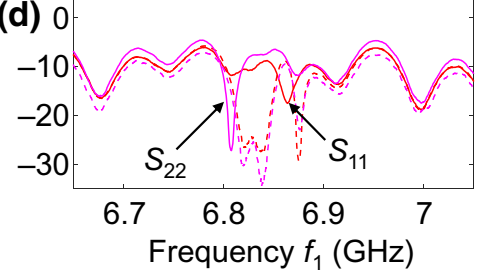

FIG. 2. Measured scattering parameters of the MPIJIS. (a) Transmission parameters, i.e., $\left|S_{21}\right|^{2}$ (blue) and $\left|S_{12}\right|^{2}$ (orange), and (b) reflection parameters, i.e., $\left|S_{11}\right|^{2}$ (red) and $\left|S_{22}\right|^{2}$ (magenta), versus signal frequency for a pump applied to $P_{1}$ at $f_{p}=2.758 \mathrm{GHz}$. The solid and dashed curves correspond to the pump being on and off, respectively. (c),(d) Same as (a),(b) except the pump is applied to $P_{2}$ instead.
Since the JPCs in the MPIJIS are operated in frequencyconversion mode without photon gain, they are not required to add noise to the processed signal $[45,46]$. However, any noise added by the MPIJIS is primarily set by its insertion loss in the forward direction, e.g., $\left|S_{i j}\right|^{2}$ (assuming $j \rightarrow i$ ), and is given by $n_{\text {add }}=\left(1-\left|S_{i j}\right|^{2}\right) / 2\left|S_{i j}\right|^{2}$, where $n_{\text {add }}$ is the number of noise-equivalent input photons at the signal frequency [35]. For the measurement in Fig. 2, which exhibits attenuation of about $2 \mathrm{~dB}$ in the forward direction, we get $n_{\text {add }}=0.29$. In the ideal case (i.e., with attenuation of $0.5 \mathrm{~dB}$ ), this figure is expected to be much lower, $n_{\text {add }}=0.06$.

\section{B. Pump power dependence}

In Fig. 3, we report, for a fixed pump frequency and fluxes (for which $p=0$ ), measurements of $\left|S_{21}\right|^{2}$ and $\left|S_{12}\right|^{2}$ of the MPIJIS as we vary the pump power. Figures 3(a) and 3(b) show measurements of $\left|S_{21}\right|^{2}$ and $\left|S_{12}\right|^{2}$ when $P_{1}$ is driven (i.e., directionality $1 \rightarrow 2$ ), whereas Figs. 3(c) and 3(d) show measurements of the same transmission parameters when $P_{2}$ is driven instead (i.e., directionality $2 \rightarrow 1$ ). The colored solid curves represent measured data, whereas the dashed black curves represent a calculated response of the device using the theoretical model presented in Appendix B. As seen in these measurements, the device response varies with the pump power in a monotonic and stable manner similar to the observed response of JPCs [41] and JPAs [47]. Other important characterization and measurement results for the MPIJIS can be found in Appendix I.

\section{Flux parity dependence}

In Appendix $G$ we present experimental data that demonstrate the dependence of the MPIJIS transmission or isolation direction on the parity of the magnetic fluxes threading its two JRMs. In particular, we show that the MPIJIS directionality can be reversed for the same pump by changing the orientation parity of the applied fluxes. This effect could allow, for example, the detection of the orientation parity of pairs of weak magnetic sources using microwave transmission measurements (see Appendix K).

\section{QUBIT READOUT MULTICHIP MODULE}

After successfully demonstrating the operation of the MPIJIS as a stand-alone device, we now report integration of it with other microwave components to form a QRMCM devoid of magnetic materials and strong magnetic fields. An image of the QRMCM and a block diagram of its components and the setup are shown in Figs. 4(a) and 4(b), respectively. It integrates a Purcell filter [35,48], a superconducting wideband directional coupler [35], two MPIJIS devices (MPIJIS 2 was damaged during wirebonding and, therefore, is not operational in this work), and a reconfigurable directional Josephson device that is identical to the MPIJIS circuit but does not include an on-chip pump hybrid, thus enabling us to operate it as a MPIJDA or a MPIJIS, depending on the applied pump frequency. The QRMCM has a few pump ports used for powering the Josephson devices. While only one pump is used for operating the MPIJIS, the reconfigurable device requires for its operation two same-frequency pumps, whose phase difference between $P_{3}$ and $P_{4}$ is $\pm \pi / 2$. The QRMCM has three main ports connecting to the readout input line, the quantum chip (containing a qubit coupled to a readout resonator), and the readout output line. The output line used here includes a commercial low-pass filter with a cutoff frequency of $8 \mathrm{GHz}$ at the base stage, a HEMT amplifier at the 4-K stage, and a superconducting $\mathrm{Nb}$-Ti coaxial cable connecting the two stages. The QRMCM setup also includes several auxiliary lines (such as IN2, IN1', and OUT1') and auxiliary components (such as a commercial directional coupler on the output line and a cryogenic circulator on the IN1' line) as seen in Fig. 4(b) (and the detailed setup diagram in Fig. 18) that allow us to probe the transmission through the QRMCM in both directions at various working points. We also use a separate input line to inject the qubit pulses, as seen in Fig. 4(b), that directly connects to the qubit chip. All other auxiliary ports of the directional Josephson devices (e.g., those that are coupled to their internal modes) are terminated with cryogenic $50-\Omega$ loads.

Using the QRMCM, we conduct four main qubit experiments, whose results are shown in Figs. 5-7 (taken in the same cooldown) and Figs. 8(b), 8(c), and 9 [taken 


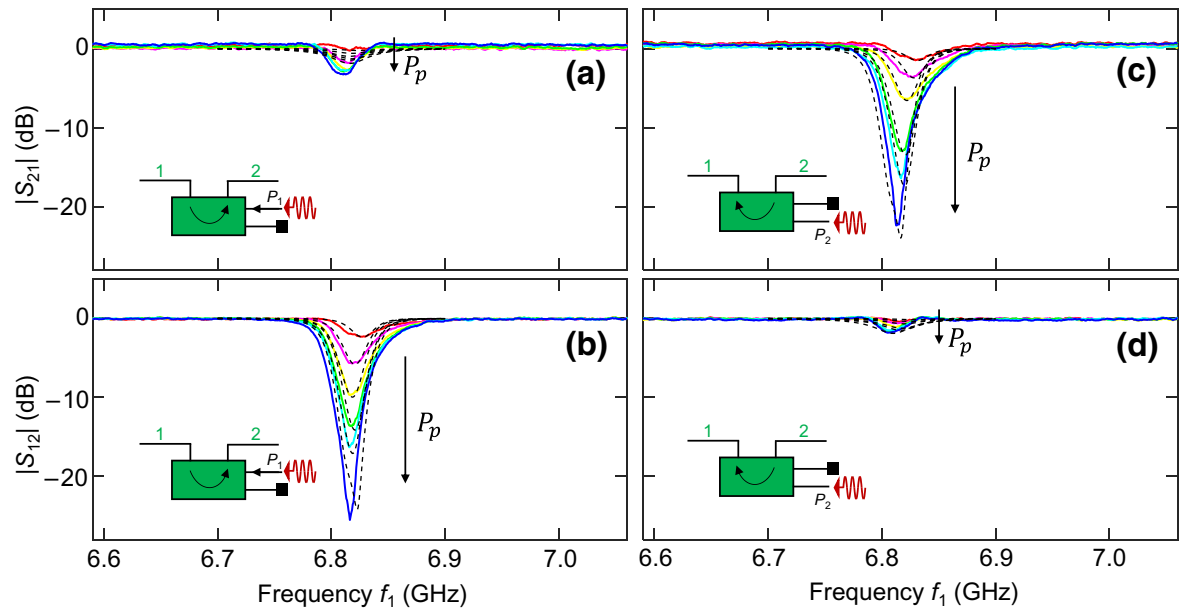

FIG. 3. MPIJIS transmission versus pump power. Transmission parameters measured versus signal frequency for various pump powers applied at $f_{p}=$ 2.727 GHz. (a),(b) Measured transmission parameters $\left|S_{21}\right|^{2}$ and $\left|S_{12}\right|^{2}$ versus frequency for various pump powers applied to $P_{1}$. (c),(d) Same as (a),(b) obtained for various pump powers applied to $P_{2}$. The dashed black curves represent a calculated response of the MPIJIS. The calculation sequence and the parameters used are given in Appendix B. in a separate cooldown, whose setup diagram is shown in Fig. 8(a)]. In attempt to optimize the distribution of the attenuation and filtering on the various lines, we perform two additional cooldowns of the QRMCM. The results of these brief cooldowns are not shown here but are fully consistent with the reported data.

In the qubit measurements in the first three experiments (i.e., Figs. 5-7), the readout pulse duration and the integration time are $0.75 \mu \mathrm{s}$. In the fourth experiment, comparing the QRMCM with magnetic isolators (i.e., Fig. 9), these times are set to $1.5 \mu \mathrm{s}$.

\section{A. MPIJIS-MPIJDA experiment}

In this experiment, we perform a fast dispersive readout measurement with high fidelity using the
QRMCM, while maintaining long coherence times for the qubit.

We operate the first MPIJIS $\left(I_{1}\right)$ of the QRMCM as an isolator and the reconfigurable directional device as a near-quantum-limited amplifier (A) (i.e., MPIJDA). In Fig. 5, we present the main results, measured for the four possible configurations of the QRMCM specified in the headings of Fig. 5(a)-5(d). In the first column, we present the forward and backward transmission parameters of the QRMCM measured between ports $1^{\prime}$ and 2 as defined in Fig. 4(b). In the second column, we present the measured readout fidelity histograms and the corresponding assignment fidelity. In the third and fourth columns, we show the measured qubit $T_{1}$ and $T_{2 E}$, respectively. The dephasing time in the various cases is calculated with the relation $T_{\varphi}^{-1}=T_{2 E}^{-1}-\left(2 T_{1}\right)^{-1}$. (a)

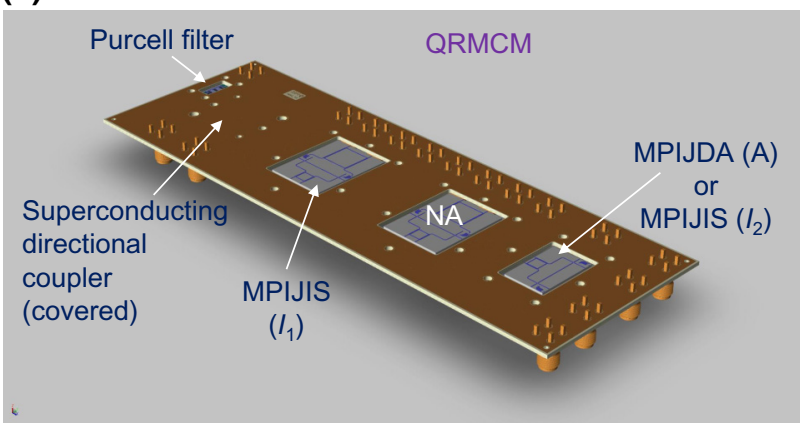

(b)

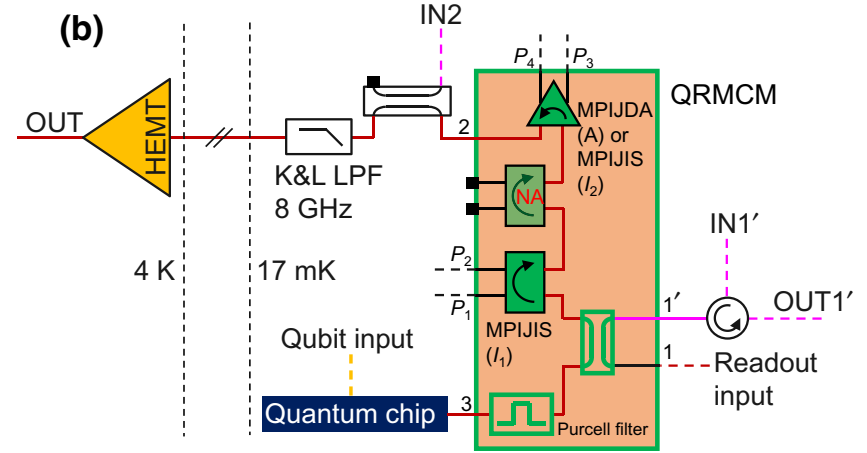

FIG. 4. (a) Image of the QRMCM featuring a printed circuit board that integrates several superconducting microwave components: a Purcell filter, a superconducting directional coupler, two MPIJIS devices [one of which is not applicable (NA)], and a reconfigurable MPIJDA (A) or MPIJIS $\left(I_{2}\right)$ device. The length and width of the QRMCM are $19.69 \mathrm{~cm}$ and $6.73 \mathrm{~cm}$, respectively. (b) Simplified schematic of the experimental setup used to obtain the measurement results shown in Figs. 5-7. A more detailed schematic of the setup is shown in Fig. 18. The QRMCM has three main ports that connect to the readout input line, the quantum chip, and the readout output line. The pump is fed to $I_{1}$ via one input line $\left(P_{1}\right.$ or $\left.P_{2}\right)$ and to the reconfigurable device via two input lines $\left(P_{3}\right.$ and $\left.P_{4}\right)$. The isolated port of the directional coupler denoted $1^{\prime}$ is used to measure the transmission through the directional devices of the QRMCM in the forward and backward directions. All other auxiliary ports are terminated by $50-\Omega$ loads. The signal path outlined in red represents the qubit readout path. The magenta lines represent auxiliary input and output lines that enable the QRMCM transmission to be probed in the forward and backward directions. A K\&L low-pass filter (LPF) with a cutoff frequency at $8 \mathrm{GHz}$ is incorporated between the QRMCM and the HEMT. 


\section{$l_{1}$ off, A off}

(a)
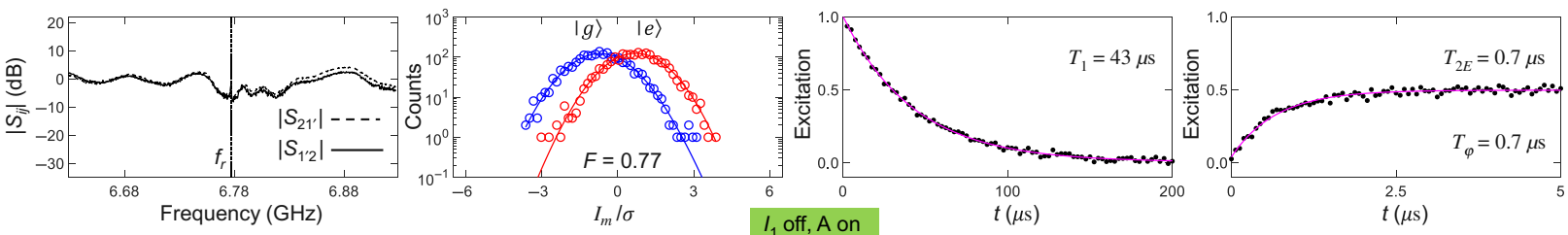

(b)
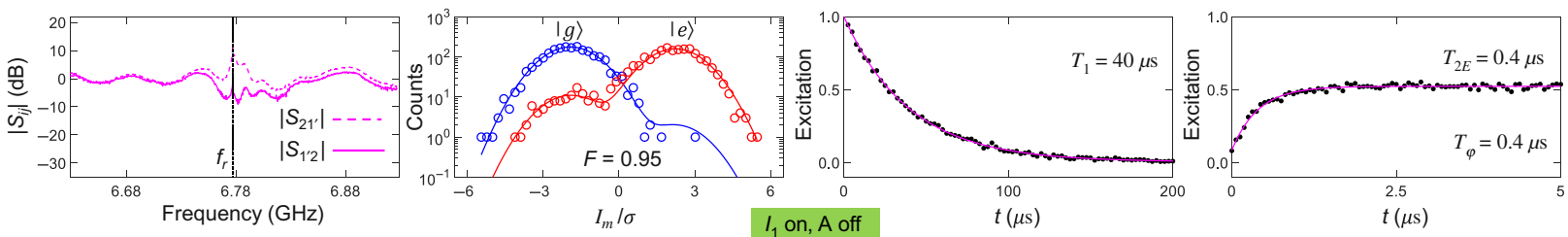

(c)
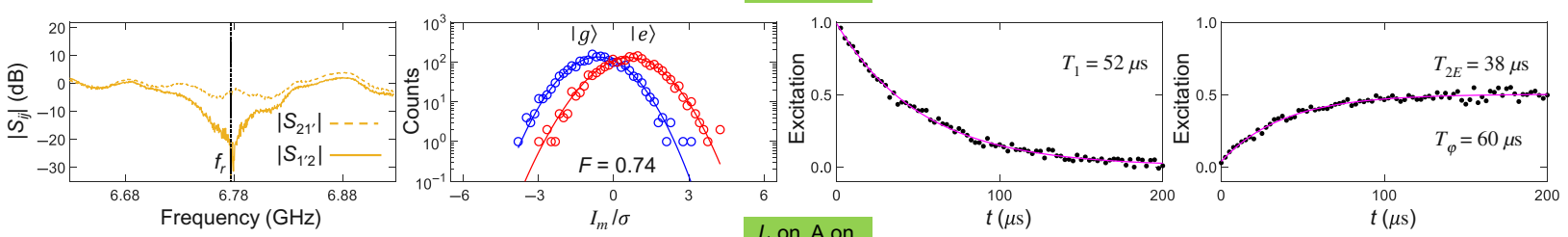

(d)
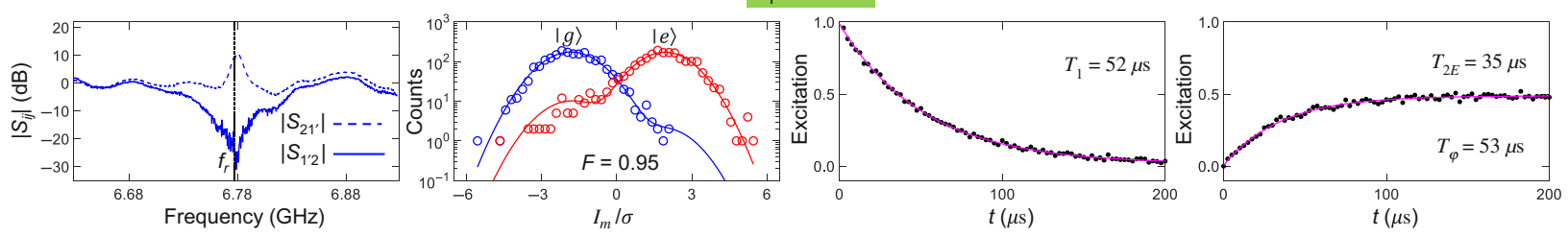

FIG. 5. High-fidelity qubit readout without cryogenic magnetic circulators and isolators. A simplified (detailed) version of the experimental setup used to obtain the dataset is shown in Fig. 4(b) (Fig. 18). From left to right, the forward $\left(\left|S_{21^{\prime}}\right|^{2}\right)$ and backward $\left(\left|S_{1^{\prime} 2}\right|^{2}\right)$ transmission parameters of the QRMCM are shown, followed by respective measurements of the readout fidelity, $T_{1}$, and $T_{2 E}$. The measurements shown in (a)-(d) correspond to different MPIJIS $_{1}\left(I_{1}\right)$ and MPIJDA (A) configurations outlined in the green headings. The vertical dashed black line in the plot on the left in (a)-(d) indicates the location of the readout frequency.

When both $I_{1}$ and A are off [Fig. 5(a)], $\left|S_{21^{\prime}}\right|^{2}$ and $\left|S_{1^{\prime} / 2}\right|^{2}$ overlap and exhibit an insertion loss of about $2 \mathrm{~dB}$ at the readout frequency. We measure a readout fidelity $F$ of $0.77, T_{1}=43 \mu \mathrm{s}$, and $T_{2 E}=T_{\varphi}=0.7 \mu \mathrm{s}$. Turning on A while keeping $I_{1}$ off [Fig. 5(b)] results in about $12 \mathrm{~dB}$ of gain in the forward direction and $-1 \mathrm{~dB}$ in the backward direction, an increase in the fidelity to 0.95 , and a slight reduction in in the coherence times, $T_{1}=40 \mu \mathrm{s}$ and $T_{2 E}=$ $T_{\varphi}=0.4 \mu \mathrm{s}$, due to excess backaction noise of the MPIJDA. Turning on $I_{1}$ while A is off [Fig. 5(c)] results in large isolation of more than $30 \mathrm{~dB}$ in the backward direction and insertion loss of about $4 \mathrm{~dB}$ in the forward direction, a similar fidelity, $F=0.74$, as in the off-off case [Fig. 5(a)], but a significant increase of the coherence times, $T_{1}=52 \mu \mathrm{s}$, $T_{2 E}=38 \mu \mathrm{s}$, and $T_{\varphi}=60 \mu \mathrm{s}$, due to the increased qubit protection against output noise. Lastly, turning both $I_{1}$ and A on [Fig. 5(d)], results in a forward gain of about $10 \mathrm{~dB}$, reverse isolation of about $22 \mathrm{~dB}$, a high readout fidelity similar to that in the off-on case [Fig. 5(b)] of $0.95, T_{1}=52 \mu \mathrm{s}$ [similar to the on-off case, Fig. 5(c)], and only a slight drop in $T_{2 E}=35 \mu \mathrm{s}$ and $T_{\varphi}=53 \mu \mathrm{s}$ compared with the on-off case [Fig. 5(c)], for which only $I_{1}$ is on.

\section{B. MPIJIS-MPIJIS experiment}

Next we operate the reconfigurable directional Josephson device in the QRMCM as a second MPIJIS $\left(I_{2}\right)$ in series with $I_{1}$ and measure the impact of added isolation on the qubit coherence. The main results of this experiment are shown in Fig. 6. Similarly to the MPIJIS-MPIJDA experiment, we measure for the four configurations of $I_{1}$ and $I_{2}$ (being on or off), outlined in the headings of Figs. 6(a)-6(d), the forward $\left|S_{21^{\prime}}\right|^{2}$ and backward $\left|S_{1^{\prime} 2}\right|^{2}$ transmission through the QRMCM plotted in the first column from the left, the readout fidelity $F$ plotted in the second column, and the qubit coherence times $T_{1}$ and $T_{2 E}$ plotted in the third and fourth columns, respectively.

With both MPIJIS devices off [Fig. 6(a)], we measure an insertion loss (through $I_{1}$ and $I_{2}$ ) of about $3 \mathrm{~dB}$ at $f_{r}$, $F=0.85, T_{1}=40 \mu \mathrm{s}$, and $T_{2 E}=T_{\varphi}=0.6 \mu \mathrm{s}$. Turning on $I_{2}$ alone [Fig. 6(b)], results in $18 \mathrm{~dB}$ of isolation at $f_{r}$ and $8 \mathrm{~dB}$ of insertion loss in the forward direction, a drop in $F$ to 0.75 , and an increase in the qubit coherence times, $T_{1}=50 \mu \mathrm{s}, T_{2 E}=3.6 \mu \mathrm{s}$ [sixfold enhancement compared with the off-off case, Fig. 6(a)], and $T_{\varphi}=3.7 \mu \mathrm{s}$. Turning on $I_{1}$ and turning off $I_{2}$ [Fig. 6(c)] yields $31 \mathrm{~dB}$ of isolation and $5 \mathrm{~dB}$ of insertion loss, $F=0.77$, a full 
(a)
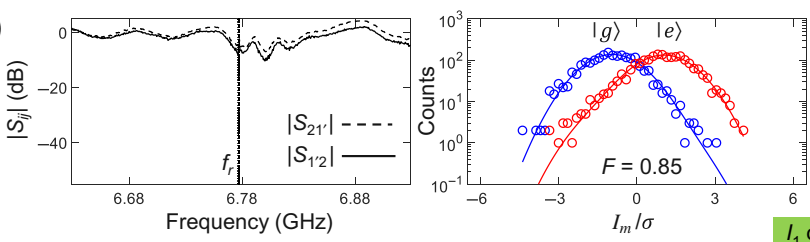

$I_{1}$ off, $I_{2}$ off

(b)
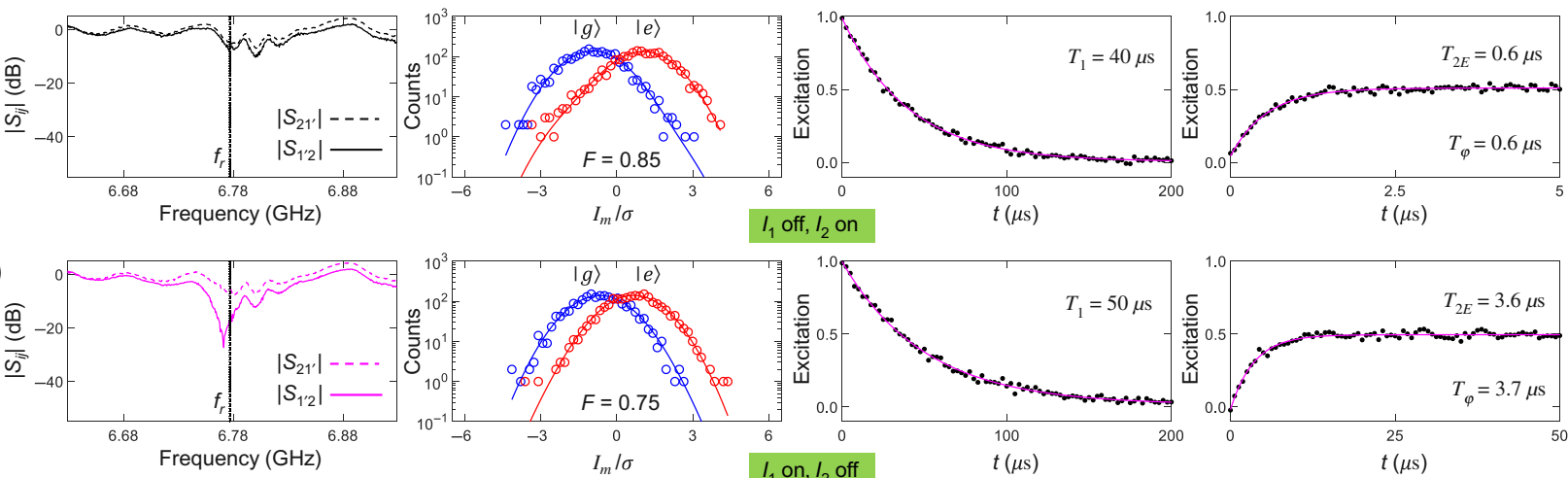

(c)
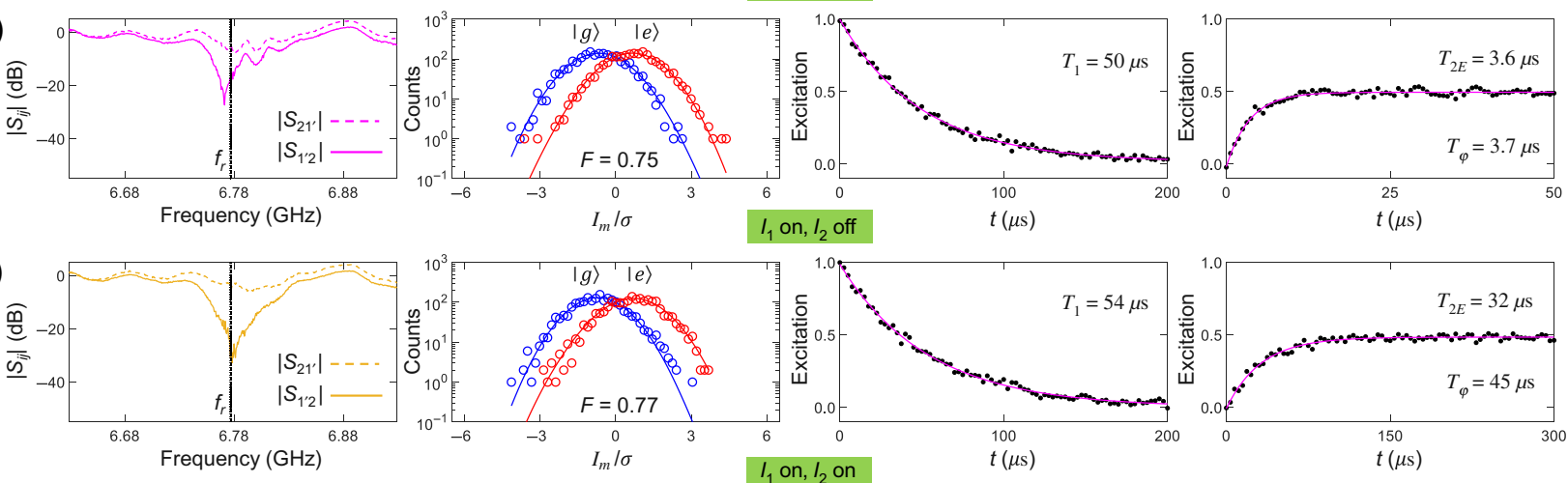

(d)
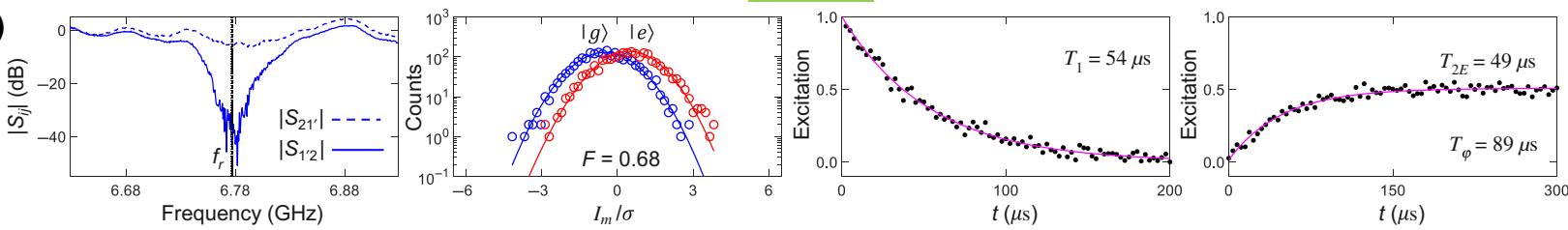

FIG. 6. Measured qubit coherence with the reconfigurable Josephson directional device in the QRMCM operated as a second interferometric Josephson isolator. A simplified (detailed) version of the experimental setup used to obtain the dataset is shown in Fig. 4(b) (Fig. 18). From left to right, the forward $\left(\left|S_{21^{\prime}}\right|^{2}\right)$ and backward $\left(\left|S_{1^{\prime} 2}\right|^{2}\right)$ transmission parameters of the QRMCM are shown, followed by respective measurements of the readout fidelity, $T_{1}$, and $T_{2 E}$. The measurements shown in (a)-(d) correspond to different MPIJIS 1 $\left(I_{1}\right)$ and MPIJIS $_{2}\left(I_{2}\right)$ configurations outlined in the green headings. The vertical dashed black line in the plot on the left in (a)-(d) indicates the location of the readout frequency.

recovery of $T_{1}\left(T_{1}=54 \mu \mathrm{s}\right.$, which falls within the qubit typical range), and a considerable increase in $T_{2 E}\left(T_{2 E}=\right.$ $32 \mu \mathrm{s}, 53$-fold higher than in the off-off case) and $T_{\varphi}=$ $45 \mu$ s. Lastly, turning on both $I_{1}$ and $I_{2}$ [Fig. 6(d)] gives an isolation of $44 \mathrm{~dB}$ at $f_{r}$ and insertion loss similar to that in the off-on case [Fig. 6(b)], $F=0.68$, and $T_{1}=54 \mu \mathrm{s}$ [similar to the on-off case, Fig. 6(c)] and a maximum in $T_{2 E}\left(T_{2 E}=49 \mu \mathrm{s}, 82\right.$-fold higher than the off-off case] and $T_{\varphi}=89 \mu \mathrm{s}$.

\section{Variable isolation experiment}

Here we investigate the dependence of the qubit coherence times on the isolator response by varying the isolation in situ via the applied pump power.

In Fig. 7(a), we plot the measured isolation curves $L_{0}(f)=\left|S_{1^{\prime} 2}(f)\right|^{2}$ of the single-pump MPIJIS as a function of the normalized pump power, where the isolation at the readout frequency $L=L_{0}\left(f_{r}\right)$ is minimal at $P_{p 0}$. In Fig. 7(b), we plot the corresponding theory fits calculated using the effective two-port model of the MPIJIS derived in Appendix B.

In Fig. 7(c), we plot the corresponding measured coherence times of the qubit (i.e., $T_{1}, T_{2 E}$, and $T_{\varphi}$ ) as a function of $L$. The dotted black line is a guide for the eye for increasing $P_{p}$. We also add on the same plot the highest coherence points obtained in the MPIJIS-MPIJIS experiment in Fig. 6(d) corresponding to $L<10^{-4}$ (not connected by the dotted line).

Since thermal photon population in the readout resonator is the likely dominant dephasing mechanism in our dispersively coupled qubit-resonator system exposed to thermal noise coming from the 4-K stage, we use the dephasing rate equation derived in Refs. [49,50], given by

$$
\Gamma_{\varphi}=\frac{\kappa}{2} \operatorname{Re}\left(\sqrt{\left(1+i \frac{\chi}{\kappa}\right)^{2}+4 i \frac{\chi}{\kappa} \bar{n}}-1\right) .
$$

In Eq. (4), $\kappa$ is total photon decay rate of the fundamental mode of the readout resonator with angular frequency $\omega_{r}=2 \pi f_{r}$ (here $\kappa$ is dominated by the rate of coupling to the external feedline $\kappa \cong \kappa_{e}$ ), $\chi$ is the qubitstate-dependent frequency shift of the readout resonator, and $\bar{n}=\Sigma_{i} F_{i} \kappa_{i} \bar{n}_{i} / \kappa$ is the average thermal photon number in the resonator [51], where $\bar{n}_{i}=1 /\left(e^{\left(\hbar \omega_{r} / k_{B} T_{i}\right)}-1\right)$ is the Bose-Einstein population of a $50-\Omega$ load noise source $i$ at effective temperature $T_{i}, \kappa_{i}$ is the readout resonator 

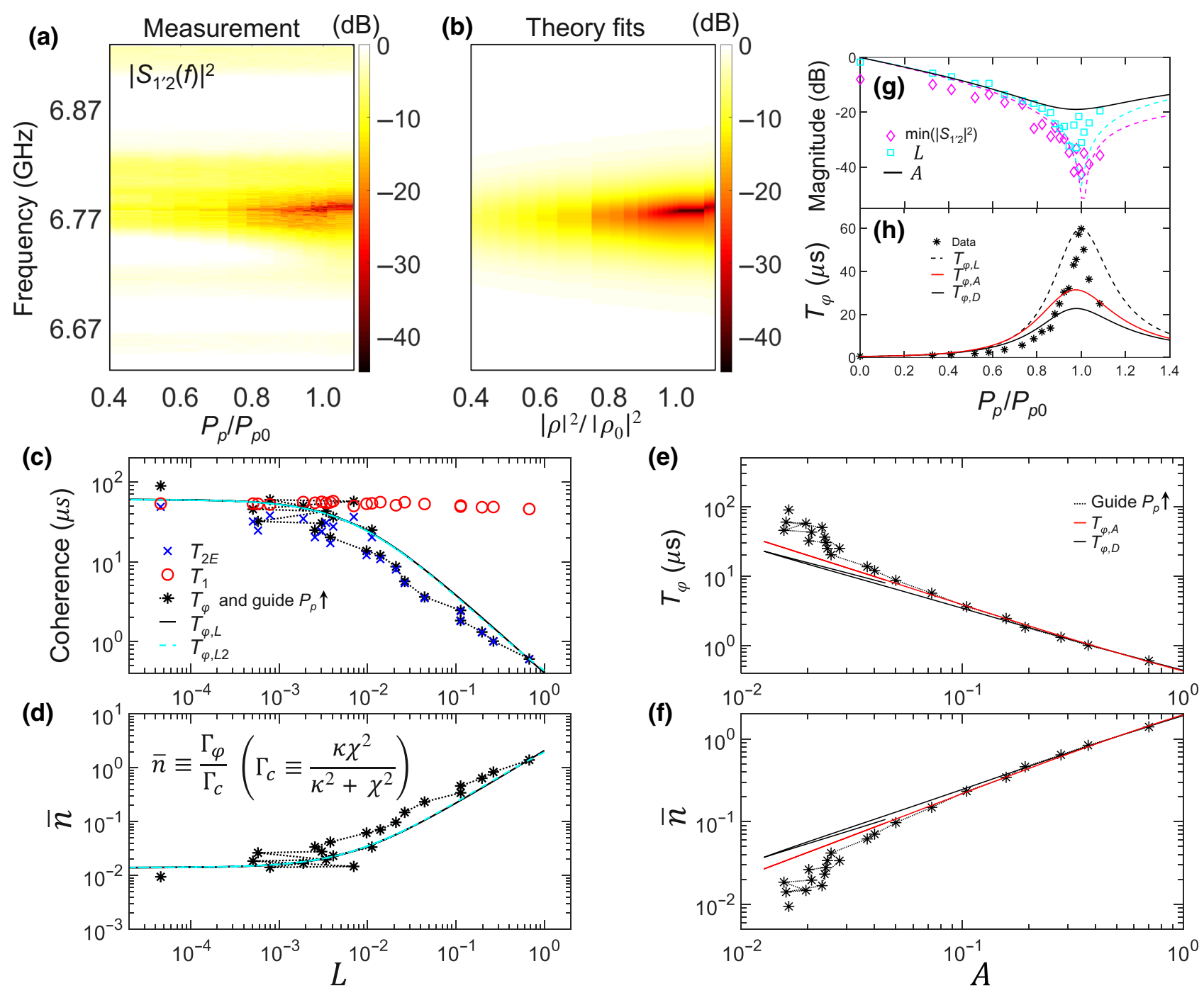

FIG. 7. Dependence of qubit coherence times on the variable isolation of the QRMCM. Measured (a) and calculated (b) isolation curves $\left|S_{1^{\prime} 2}\right|^{2}$ of the MPIJIS $\left(I_{1}\right)$ versus frequency and normalized pump power. The calculated response uses the effective two-port model of the MPIJIS presented in Appendix B, with the parameters $\gamma_{a} / 2 \pi=100 \mathrm{MHz}, \gamma_{b} / 2 \pi=140 \mathrm{MHz}, \omega_{a} / 2 \pi=6.765 \mathrm{GHz}$, $\omega_{p} / 2 \pi=2.712 \mathrm{GHz}$, and $|\alpha|=0.4$. (c) Qubit coherence times versus the isolation magnitude at the readout frequency $L$. (d) Extracted $\bar{n}$ versus $L$. The solid black and dashed cyan fits in (c),(d) are calculated using Eqs. (4) and (5), respectively. Measured $T_{\varphi}$ (e) and extracted $\bar{n}$ (f) (stars) versus the combined filtering parameter $A$ of the MPIJIS and readout resonator. The dotted black line in (c)-(f) is a guide for the eye for increasing $P_{p}$, and the data points unconnected by the dotted line correspond to the on-on configuration of the MPIJIS-MPIJIS experiment [Fig. 6(d)]. The solid red and black curves in (e),(f) represent calculated fits (see the main text for details). The black curve represents for the saturation effect of the MPIJIS due to pump depletion. (g) Measured isolation magnitude at the minimum points (diamonds) and at the readout frequency (squares) versus normalized pump power. The corresponding theory fits are plotted as dashed magenta and cyan curves. The solid black curve represents the calculated parameter $A$. (h) Measured $T_{\varphi}($ stars) versus normalized pump power. The dashed black, solid red, and sold black curves represent theory fits based on three models of the effect of the MPIJIS on $T_{\varphi}$ (see the main text for details).

relaxation rate through source $i$, and $F_{i}$ is the linear power attenuation between source $i$ and the port through which it couples to the readout resonator (e.g., qubit or readout port).

To fit the measured dephasing time, given by $T_{\varphi} \equiv$ $\Gamma_{\varphi}^{-1}$, we model the MPIJIS as a cold filter that attenuates thermal noise coming from the output chain. Considering the case of a constant filter with attenuation $L$, where $L$ is the power isolation at $f_{r}$, we express $\bar{n}$ as $\bar{n}=L \bar{n}_{o}+\bar{n}_{r}$, where $\bar{n}_{o}$ is the thermal photon number acted upon by the MPIJIS devices, while $\bar{n}_{r}$ is a residual thermal photon population due to out-of-band noise or sources that lie outside the isolation path (e.g., couple to the MPIJIS pump ports or qubit port). Substituting $\bar{n}$ in the inverse of Eq. (4), we get the black curve fit $T_{\varphi, L}$ shown in Fig. 7(c) as a function of $L$. An almost identical fit $T_{\varphi, L 2}$ corresponding 


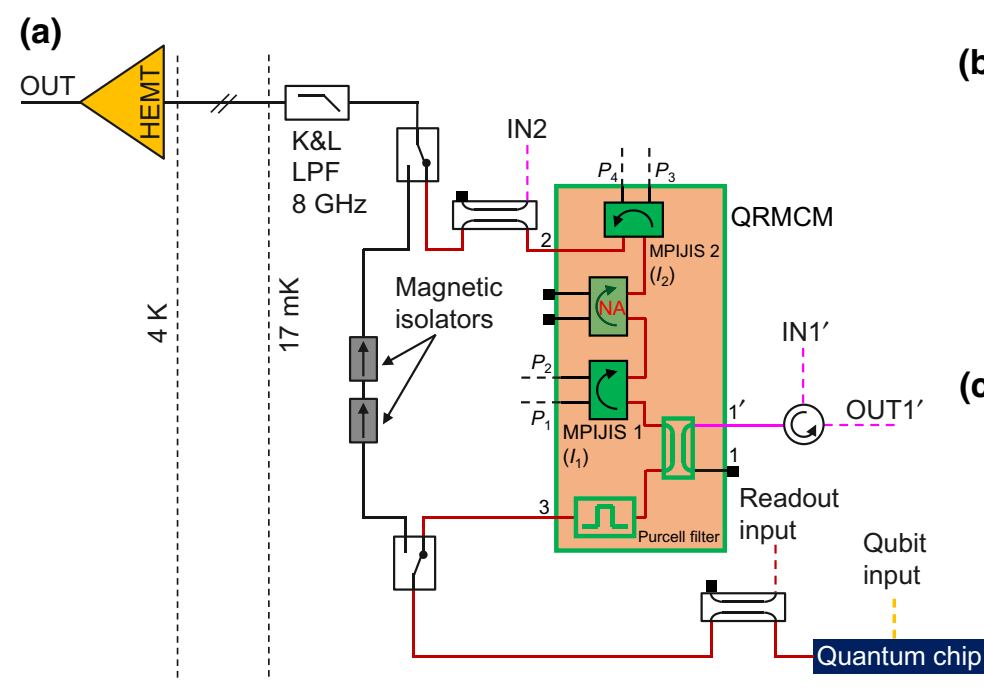

(b)

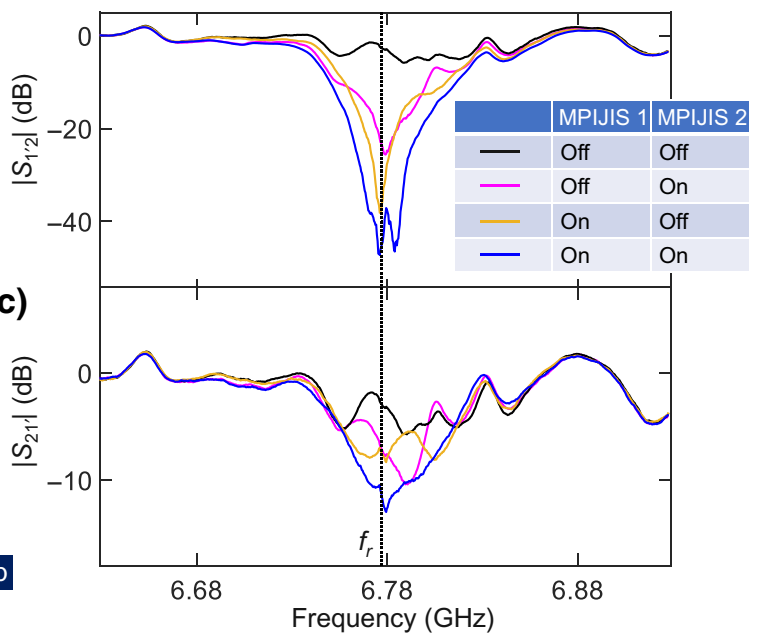

FIG. 8. Experiment comparing two cryogenic magnetic isolators and the QRMCM. (a) Simplified schematic of the experimental setup used for directly comparing, in the same cooldown, the performance of the QRMCM and that of two commercial magnetic isolators connected in series as they can be switched in and out of the qubit output chain. This setup is used to obtain the measurement results shown in Fig. 9. A more detailed schematic is shown in Fig. 20. The signal path outlined in red represents the qubit readout path with the QRMCM switched in. The magenta lines represent auxiliary input and output lines that enable the QRMCM transmission to be probed in the forward and backward directions. In the schematic, NA, not applicable and LPF, low-pass filter. (b),(c) The measured isolation $\left|S_{1^{\prime} 2}\right|^{2}$ and transmission $\left|S_{21^{\prime}}\right|^{2}$ of the QRMCM versus frequency for the different operation configurations of the QRMCM outlined in the table. The qubit coherence measurements corresponding to the three bottom configurations (in which at least one of the MPIJIS is on) are shown in Fig. 9. The vertical dashed black line in (b),(c) indicates the location of the readout frequency.

to the dashed cyan curve in Fig. 7(c) is obtained by our substituting $\bar{n}$ in the approximation of Eq. (4) (in the limit $\bar{n} \ll 1)[52]$, given by

$$
\Gamma_{\varphi}=\Gamma_{c} \bar{n}
$$

where $\Gamma_{c}=\kappa \chi^{2} /\left(\kappa^{2}+\chi^{2}\right)$. The values of $\bar{n}_{o}=2.08$ and $\bar{n}_{r}=0.014$ in these fits are set to give the dephasing times measured for $P_{p}=0$ and $P_{p}=P_{p 0}$. Similarly, in Fig. 7(d), we plot $\bar{n} \equiv \Gamma_{\varphi} / \Gamma_{c}$ measured versus $L$ (black stars) and the corresponding theory fits obtained with Eq. (4) (black) and Eq. (5) (cyan). In Figs. 7(c) and 7(d), we see that the theory fits, in this case, overestimate the dephasing time in the intermediate pump power range between 0 and $P_{p 0}$ or alternatively underestimate $\bar{n}$ in the resonator. Using $\bar{n}_{o}$ and $\bar{n}_{r}$ and the Bose-Einstein population expression $\bar{n}_{i}$, we evaluate the effective temperature of the noise source seen by the qubit in the $L=1$ and $L \ll 1$ cases, which is about $1 \mathrm{~K}$ and $70 \mathrm{mK}$, respectively.

In Figs. 7(e) and 7(f), we further analyze the dephasing time measurements. We assume that the noise comes solely through the MPIJIS path and consider the combined filtering effect, denoted $A$, of the frequency-dependent MPIJIS isolation $L_{0}(\omega)=\left|S_{12}\right|^{2}$ [using Eq. (B7)] and the Lorentzian response of the resonator given by $R(\omega)=$ $(\kappa / 2 \pi) /\left[\left(\omega-\omega_{r}\right)^{2}+(\kappa / 2)^{2}\right]$. Hence, we express $\bar{n}$ $=A \bar{n}_{\text {out }}$, where $A \equiv \int_{\omega_{c 1}}^{\omega_{c 2}} L_{0}(\omega) R(\omega) d \omega$ and $\bar{n}_{\text {out }}$ represents an effective photon number of the output-line noise in the relevant frequency range $\left[\omega_{c 1}, \omega_{c 2}\right]$.

Using this model with $\bar{n}_{\text {out }}=2.1$, we plot, in Figs. 7(e) and $7(\mathrm{f})$, the measured dephasing time and $\bar{n}$ (black stars) versus the parameter $A$, which we numerically calculate using the measured MPIJIS curves shown in Fig. 7(a). We also plot with solid red lines the corresponding theory fits for the dephasing time $T_{\varphi, A}$ and $\bar{n}$ obtained with Eq. (4) and the parameter $A$ evaluated with use of the calculated response of the MPIJIS, featured in Fig. 7(b). Similarly to Fig. 7(c), the dotted black line in Figs. 7(d)-7(f) is a guide for the eye for increasing $P_{p}$, and the data point in each figure that is not connected by the dashed line belongs to the on-on configuration of the MPIJIS-MPIJIS experiment [Fig. 6(d)]. As seen in Figs. 7(e) and 7(f), the theory fits drawn as red lines yield good agreement with the measured data in the low-to-mid pump power range $P_{p}<P_{p 0}$. Deviations up to a factor of 2 between the fits and the data are observed around and above $P_{p 0}$. Notably, we observe an increase of $T_{\varphi}$ (or decrease in $\bar{n}$ ) even for similar values of the parameter $A$ near $10^{-2}$, where it plateaus. However, as revealed by the dashed black line, higher $T_{\varphi}$, corresponding to similar $A$ values, seems to correlate with higher applied pump powers. To account for this pump power dependency, we update the parameter $A$ (as done in Appendixes D and $\mathrm{E}$ ) to include the effect of input-power saturation of the MPIJIS (i.e., dynamic 
range) due to pump depletion. Substituting the result of Eq. (E5) into the inverse of Eq. (4) with $\bar{n}_{\text {out }}=1.7$ yields the updated theory fit $T_{\varphi, D}$ drawn as a solid black curve in Fig. 7(e). Importantly, although the fits $T_{\varphi, A}$ and $T_{\varphi, D}$ drawn in Fig. 7(e) yield similar quantitative agreement with the data, $T_{\varphi, D}$ qualitatively reproduces the semi-U-turn feature of the measured dephasing time with respect to $A$. Similar characteristics are seen in the corresponding fits of $\bar{n}$ in Fig. 7(f). It is important to emphasize here the implications of the data drawn in Figs. 7(e) and 7(f). They show [see Eq. (E4)] that for similar $A$ values of the MPIJIS, which generally correspond to similar isolation response versus frequency, the qubit dephasing time increases when the ratio of the incoming noise photon per unit time to the input pump photon per unit time decreases. Furthermore, the fact that in the double-isolation experiment [represented by the top and bottom data points in Figs. 7(e) and 7(f), respectively], the measured dephasing time is higher than the maximum achieved with one isolation stage, despite having a similar value of $A$, might be due to the presence of two pump inputs feeding the two isolation stages, which further reduce the noise to pump photon ratio.

To complete the picture, we plot in Fig. 7(g) the measured isolation at the minimum points (diamonds) and at the readout frequency (squares) and the calculated parameter $A$ (solid black curve) as a function of the normalized pump power $P_{p} / P_{p 0}$. The corresponding theory fits for the isolation at the global minimum points and the readout frequency (i.e., $L$ ) are plotted as dashed magenta and cyan curves, respectively. We also plot in Fig. 7(h) along the same $x$ axis, the measured dephasing time (black stars) and the theory fits $T_{\varphi, L}$ (dashed black curve), $T_{\varphi, A}$ (solid red curve), and $T_{\varphi, D}$ (solid black curve) discussed earlier.

\section{QRMCM versus two magnetic isolators}

In this experiment, we modify the experimental setup inside the fridge to enable a direct comparison between the protection provided by the QRMCM and two commercial magnetic isolators connected in series as illustrated in Fig. 8(a), showing the main components (a detailed diagram of the setup is displayed in Fig. 20). In this setup, we connect the readout input line to a commercial directional coupler to enable the passage of the reflected readout signals off the quantum chip through the QRMCM or magnetic isolators depending on the state of a first cryogenic switch, which connects to either the Purcell filter port of the QRMCM or the input of the magnetic isolators. We also add a second cryogenic switch connected to the rest of the output line, which, depending on its state, connects to either the output of the QRMCM (i.e., the auxiliary directional coupler connected to IN2) or the output of the magnetic isolators. In both cases, the readout and qubit input lines are the same as is the output line, which includes the low-pass filter and the HEMT.

The two commercial magnetic isolators used in this comparison are 4-12-GHz isolators. Separate characterization of this kind of broadband isolator at $10 \mathrm{mK}$ in a different fridge (data not shown) shows that it gives more than $20 \mathrm{~dB}$ of isolation in the range from 1 to $12 \mathrm{GHz}$ and even stronger isolation, exceeding $60 \mathrm{~dB}$, at the readout frequency of this experiment.

In Figs. 8(b) and 8(c), we plot the transmission in the backward direction $\left|S_{1^{\prime} / 2}\right|^{2}$ and the forward direction $\left|S_{21^{\prime}}\right|^{2}$ measured through the two MPIJIS devices, i.e., $I_{1}$ and $I_{2}$, corresponding to the four different configurations listed in the inset table. Similarly to the double-isolation experiment reported in Fig. 6, we obtain at this new working point an isolation of about $45 \mathrm{~dB}$ at $f_{r}$ but a higher insertion loss in the forward direction, $10 \mathrm{~dB}$ instead of $8 \mathrm{~dB}$, which underscores the relatively large tuneup parameter space for Josephson parametric devices that possess several degrees of freedom, such as fluxes and pump drives (frequency and power). In the following section, we discuss how this tuneup parameter space can be reduced.

In Fig. 9, we plot the qubit coherence times measured with the magnetic isolator setup [Fig. 9(a)] and the QRMCM configurations [Figs. 9(b)-9(d)] specified in the headings. From left to right, we plot $T_{1}$ (relaxation), $T_{R}$ (Ramsey), $T_{E}$ (echo), and $T_{\text {CPMG }}$ [Carr-PurcellMerboom-Gill-like (CPMG-like) decoherence measurement]. An illustration of the pulse sequence applied in the different measurements is shown at the top. In Fig. 9(a), we measure for the magnetic isolator setup, $T_{1}=47 \mu \mathrm{s}$, $T_{R}=74 \mu \mathrm{s}, T_{E}=83 \mu \mathrm{s}$, and $T_{\mathrm{CPMG}}=93 \mu \mathrm{s}$. The dephasing time, $T_{\varphi, i}$, associated with the various decoherence measurements $i=R, E, \mathrm{CPMG}$, is calculated with the generalized relation $T_{\varphi, i}^{-1}=T_{i}^{-1}-\left(2 T_{1}\right)^{-1}$. From the results in Fig. 9(a), we find that in the case of two magnetic isolators, the qubit decoherence is limited mainly by low-frequency noise since $T_{R}<T_{E}<T_{\mathrm{CPMG}}$, which can be filtered out by adding one $\pi$ pulse in the echo measurement and four in the CPMG-like measurement applied here. We also achieve in this input-output line configuration the maximum attainable decoherence time, approximately equal to $2 T_{1}$ (i.e., limited by $T_{1}$ ), thus forming an ideal benchmark configuration for evaluating the QRMCM performance.

We turn now to the coherence results obtained with the QRMCM shown in Figs. 9(b)-9(d). In the baseline case, where both MPIJIS devices, $I_{1}$ and $I_{2}$, are off (data not shown), we obtain $T_{1}=28 \mu \mathrm{s}, T_{R}=0.7 \mu \mathrm{s}$, and $T_{E}=0.6 \mu \mathrm{s}$. In Fig. 9(b), where only $I_{2}$ is on, we obtain $T_{1}=41 \mu \mathrm{s}, T_{R}=21 \mu \mathrm{s}, T_{E}=22 \mu \mathrm{s}$, and $T_{\mathrm{CPMG}}=26 \mu \mathrm{s}$. Similarly, in Fig. 9(c), where only $I_{1}$ is on, we obtain $T_{1}=42 \mu \mathrm{s}, T_{R}=13 \mu \mathrm{s}, T_{E}=29 \mu \mathrm{s}$, and $T_{\mathrm{CPMG}}=36 \mu \mathrm{s}$. Lastly, in Fig. 9(d), where both $I_{1}$ and $I_{2}$ are on, we measure the longest coherence times, i.e., $T_{1}=45 \mu \mathrm{s}, T_{R}=$ $30 \mu \mathrm{s}, T_{E}=46 \mu \mathrm{s}$, and $T_{\mathrm{CPMG}}=52 \mu \mathrm{s}$. While this case 
(a)
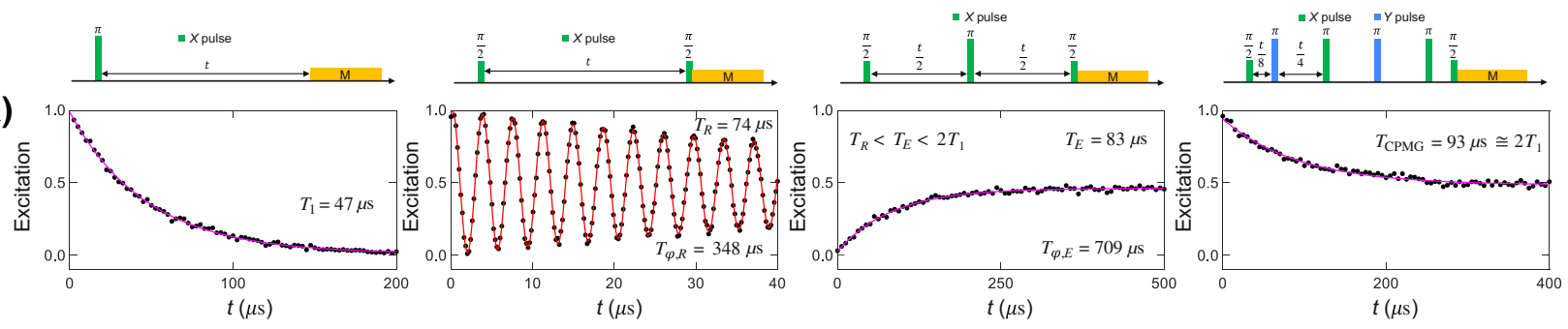

(b)
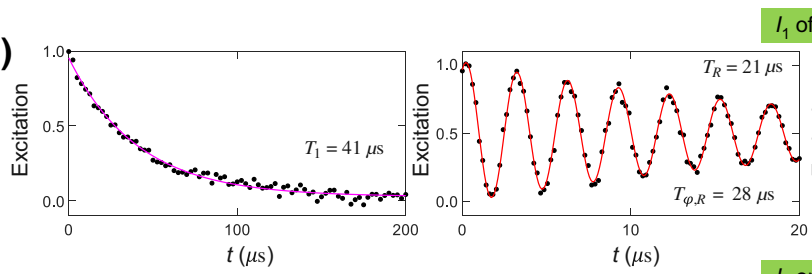

$I_{1}$ off, $I_{2}$ on

(c)
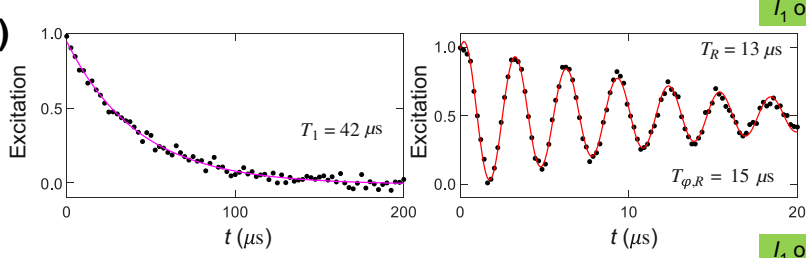

$l_{1}$ on, $I_{2}$ off
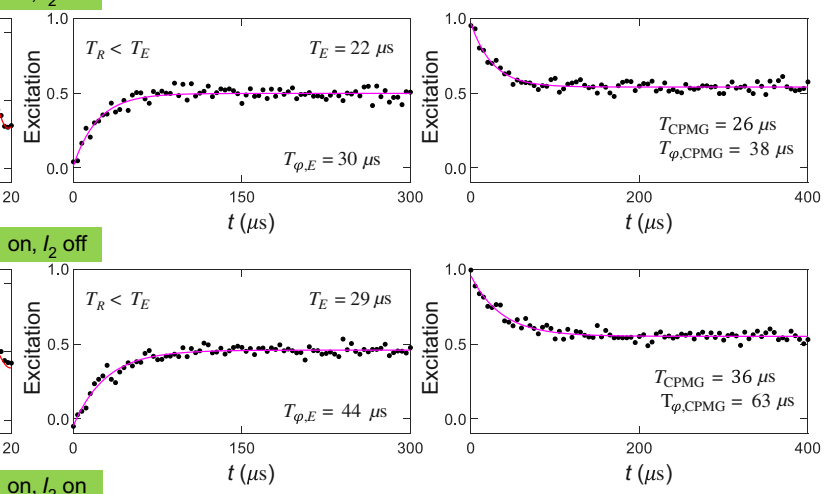

(d)
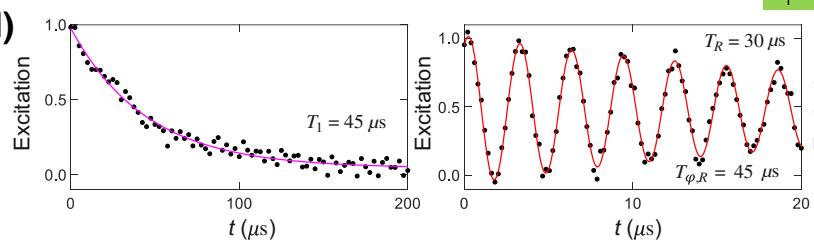

on, $I_{2}$ on $\quad t(\mu \mathrm{s})$
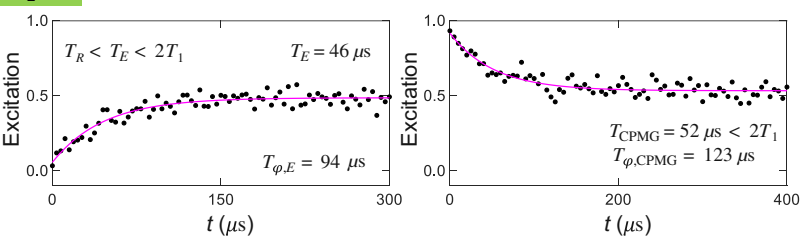

FIG. 9. Comparison of the qubit coherence times measured using two cryogenic magnetic isolators versus the QRMCM. (a) Qubit coherence measurements taken with the output chain incorporating two commercial wideband magnetic isolators. (b)-(d) Similar coherence measurements taken in the same cooldown with the output chain incorporating the QRMCM. A simplified (detailed) version of the experimental setup used to obtain the dataset is shown in Fig. 8(a) (Fig. 20). From left to right $T_{1}, T_{R}, T_{E}$, and $T_{\mathrm{CPMG}}$ measurement results are shown. The pulse sequence used in the various cases is shown at the top. The measurements shown in (b)-(d) correspond to different MPIJIS $1\left(I_{1}\right)$ and MPIJIS $2\left(I_{2}\right)$ configurations outlined in the green headings. The corresponding QRMCM transmission parameters measured for these configurations are shown in Figs. 8(b) and 8(c).

gives $T_{1}=45 \mu \mathrm{s}$, which is effectively equal to $47 \mu \mathrm{s}$ obtained with the two-magnetic-isolator setup, and yields a considerable increase in the decoherence times with the addition of $\pi$ pulses as indicated by $T_{R}<T_{E}<T_{\mathrm{CPMG}}$, it achieves $T_{E}$ and $T_{\mathrm{CPMG}}$ that are only slightly higher than $T_{1}$ and much lower than $2 T_{1}$. This result suggests that in the QRMCM case, the decoherence time is likely limited by high-frequency noise, such as residual thermal photon noise in the readout resonator that cannot be filtered out by applying a small number of $\pi$ pulses [53].

\section{DISCUSSION}

The qubit-QRMCM experiment differs from the qubit-MPIJIS experiment reported in Ref. [35] in several important aspects: it (1) realizes an on-chip MPIJIS device operated with a single pump; it (2) introduces a working QRMCM operated in continuous mode, which integrates a
Purcell filter, a superconducting directional coupler, MPIJIS devices, and a reconfigurable MPIJDA or MPIJIS device; (3) it demonstrates a high-fidelity, high-coherence, dispersive qubit measurement without any magnetic isolators and circulators in the output chain; (4) it achieves an isolation of more than $40 \mathrm{~dB}$ at the readout frequency using two MPIJIS devices in series; and (5) it can be used to investigate the dependence of the qubit coherence on the MPIJIS response, varied in situ with use of the pump tone.

In addition to enabling high-fidelity dispersive readout, the QRMCM scheme presented here has two main advantages: (1) it is fully compatible with frequency-multiplexed readout, which is useful in scalable architectures (provided that the bandwidth and saturation power of the MPIJIS or MPIJDA can be significantly increased, as we discuss below); (2) its isolation and amplification stages are inherently compatible due to their shared circuitry, fabrication process, and mode of operation. This compatibility could 
(a)

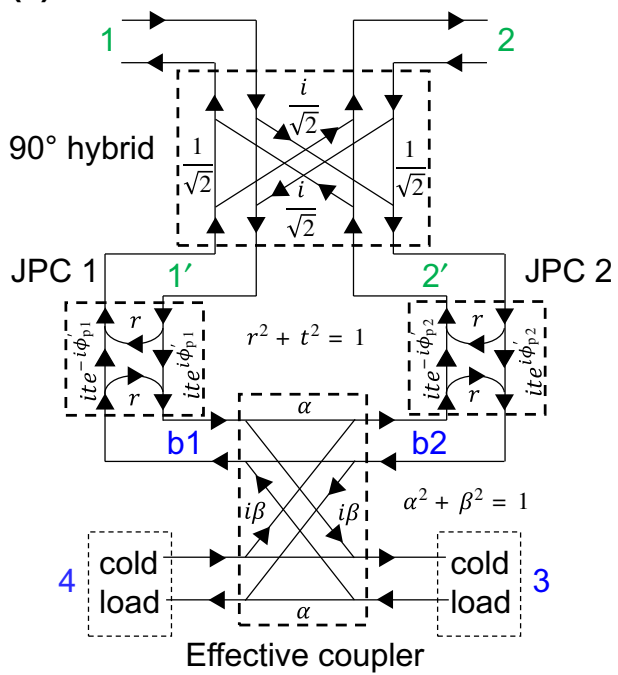

(b)

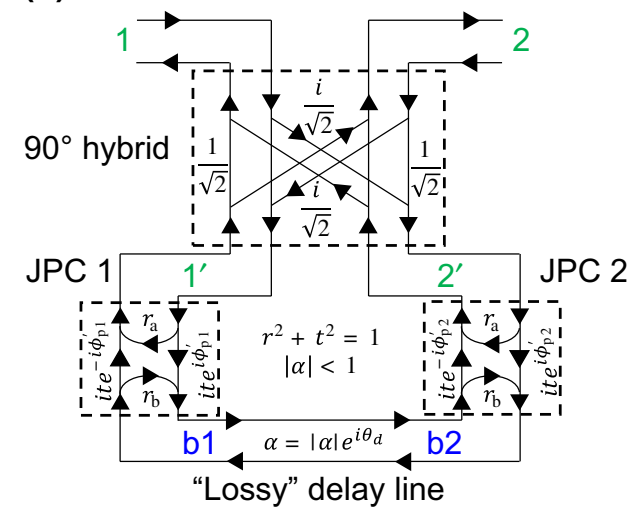

FIG. 10. Signal flow graphs for the MPIJIS. The graphs in (a),(b) exhibit two JPCs operated in frequency-conversion mode on resonance, i.e., $f_{1}=f_{a}$ and $f_{2}=f_{b}$. Ports a of the JPCs, denoted as " 1 '" and " 2 '," are coupled via a $90^{\circ}$ hybrid, while their internal b ports, denoted as "b1" and "b2", are coupled via a fictitious coupler in (a) or equivalently a lossy delay line in (b), which model the attenuation present in the internal $\mathrm{b}$ channel of the MPIJIS due to dissipation in the $50-\Omega$ cold terminations. In (a) the coupler coefficients $\alpha$ and $\beta$ are taken to be real, satisfying the condition $\alpha^{2}+\beta^{2}=1$. The signals transmitted between ports a and $\mathrm{b}$ of the JPC undergo frequency conversion and acquire a nonreciprocal phase shift, which depends on the phase of the drive.

be particularly useful in large systems, which benefit from standardization, reliability, and matching.

The measured response of the MPIJIS shown in Figs. 2 and 3 differs from the ideal theoretical case in two notable aspects. The first is the MPIJIS attenuation on resonance in the forward direction of about $2 \mathrm{~dB}$, which is higher than the $0.5 \mathrm{~dB}$ predicted for a device whose JPCs are operated around the 50:50 beam splitting point, and whose modes $\mathrm{b}$ are equally coupled to each other and to the cold terminations. The likely cause of this effect, as revealed from the calculated response in Fig. 3, is that modes b of the JPCs are coupled more strongly to the cold terminations than to each other (see Appendix B). Consequently, this suggests it is possible to reduce the forward attenuation of the device by adjusting the unintentional asymmetry in the couplings in future designs. The second is the slight frequency detuning between the dips in the reflection [Figs. 2(b) and 2(d)] and transmission [Figs. 2(a) and 2(c)] parameters when the MPIJIS is on. This effect could be due to a phase imbalance in the signal hybrid, which results in a slightly different frequency condition for constructive and destructive interferences in the two cases.

The higher insertion loss $(2-3 \mathrm{~dB})$ of the MPIJIS and MPIJDA devices in the off state, seen, for example, in Figs. 5(a), 6(a), 8(b), and 8(c), originates from mismatches between the resonances of their JPC building blocks at the given flux-biasing points and the phase and amplitude imbalance of their signal hybrids, both of which can be minimized to about $0 \mathrm{~dB}$ [as seen, for example, in
Figs. 2(a) and 2(c) for the stand-alone device biased at a higher frequency] by increasing the uniformity of their JPCs, operating them near their maximum frequencies versus flux, and better match of the center frequency of their signal hybrids to the intended operation frequency.

Following this work, there are several avenues to explore going forward: (1) Realizing a single-pump, onchip MPIJDA, which uses an adapted hybrid for the pump in an analogous manner to the single-pump MPIJIS. (2) Pining down the source of the residual high-frequency noise limiting $T_{2 E}$ in the QRMCM case in comparison with the conventional magnetic isolator setup (see Fig. 9). In particular, it should be investigated whether it originates from out-of-band noise coming from the output chain (i.e., lies outside the narrow bandwidth of the MPIJIS devices) or from incoming noise that lies outside the isolation path of the MPIJIS devices (e.g., thermal noise entering through the pump-line circuitry). (3) Reducing the size of the QRMCM components using lumped-element implementations of the JPCs [54] and hybrids [55]. (4) Increasing the bandwidth and saturation power of the MPIJIS and MPIJDA to support multiplexed qubit readout in scalable architectures. This could potentially be achieved by first increasing the bandwidth and saturation power of JPCs, which constitute the bottleneck. One possible way to increase the JPC bandwidth is by implementation of an impedance-matching network between its Josephson junctions and external feedlines, as was successfully demonstrated in the case of single-port Josephson 
parametric amplifiers [56,57]. Increasing the saturation power of JPCs, on the other hand, requires getting a better handle on the Kerr and higher-order nonlinearities exhibited by the device [58-60], which are dependent on the ratios of the inductances of the Josephson junctions to the linear inductances inside and outside the ring [59]. For example, the analysis of Ref. [59] shows that there exists an experimentally feasible inductance-ratio parameter space in which JPCs have saturation powers as high as $-104 \mathrm{dBm}$.

Furthermore, increasing the bandwidth of these directional Josephson devices and reducing their footprint are expected to yield another benefit: reducing their tuneup parameter space, by further relaxing the matching requirement of their JPCs and enabling them to be flux-biased using one coil or flux line versus two. This will further simplify and decrease the variability of their tuneup procedure, and reduce the number of flux lines and sources needed per device.

Finally, it is important to emphasize that the QRMCM concept introduced here constitutes an interface block between the qubit system and the input and output lines that is modular and versatile, and, therefore, it is not limited to specific superconducting components or readout schemes. For example, the QRMCM could potentially serve in future readout schemes that rely on single microwave photon detectors [61-63] instead of parametric amplifiers.

\section{CONCLUSION}

We realize a QRMCM devoid of magnetic materials and strong magnetic fields. The QRMCM includes a Purcell filter, a superconducting directional coupler, two MPIJIS devices, and one reconfigurable directional Josephson device integrated into one PCB. We use the QRMCM alongside an off-the-shelf low-pass filter connected at its output to read out a superconducting qubit without any cryogenic magnetic isolators or circulators in the output chain. With the reconfigurable device operated as a MPIJDA and one of the MPIJIS devices turned on, we demonstrate a fast $\left(T_{r}=0.75 \mu \mathrm{s}\right)$, high-fidelity $(F>$ $0.95)$, dispersive measurement of a coherent qubit with $T_{1}=52 \mu \mathrm{s}$ and $T_{2 E}=35 \mu \mathrm{s}$. We further increase the qubit coherence time, $T_{2 E}$, to $49 \mu \mathrm{s}$ by operating the reconfigurable device as a second MPIJIS, with a total QRMCM isolation of $45 \mathrm{~dB}$ at the readout frequency and a bandwidth of about $13 \mathrm{MHz}$. Moreover, by varying the isolation of the QRMCM with the pump power, we demonstrate an in situ increase of $T_{2 E}$ and $T_{\varphi}$ by a factor of 80 and 150 , respectively (up to a maximum of $T_{2 E}=49 \mu \mathrm{s}$ and $T_{\varphi}=89 \mu \mathrm{s}$ ). A direct comparison with an output chain that includes two commercial wideband magnetic isolators in the output chain and achieves $T_{2 E} \approx 2 T_{1}$ shows that the qubit dephasing time measured with the QRMCM (for which $T_{2 E} \approx T_{1}=45 \mu \mathrm{s}$ ) is likely limited by the residual thermal photon population in the readout resonator that is not acted upon by the MPIJIS devices.

One key component enabling these results is the single-pump, on-chip MPIJIS realized in this work, which comprises two nominally identical nondegenerate, threewave Josephson mixers that are coupled in an interferometric setup and operated in frequency-conversion mode. The microwave drive, giving rise to nonreciprocity, is fed through an on-chip quadrature hybrid, which equally splits the drive between the mixers and imposes the required phase difference (i.e., $\pm \pi / 2$ ) between the split drives. The MPIJIS yields on-resonance attenuation of about $2 \mathrm{~dB}$ in the forward direction and $23 \mathrm{~dB}$ in the backward direction with a dynamical bandwidth of $8 \mathrm{MHz}$. The device has a tunable bandwidth of about $300 \mathrm{MHz}$ with isolation larger than $18 \mathrm{~dB}$ and input saturation power of about $-120 \mathrm{dBm}$ at $21 \mathrm{~dB}$ of isolation (see Appendix I).

An improved and smaller version of this QRMCM that integrates large-bandwidth and high-saturation MPIJIS and MPIJDA devices could enable frequency-multiplexed readout of multiple qubits in scalable quantum processor architectures.

\section{ACKNOWLEDGMENTS}

B.A. expresses gratitude to Jerry M. Chow and Pat Gumann for enabling this work. Fruitful discussions with David Lokken-Toyli, Luke Govia, Ted Thorbeck, and Oliver Dial are highly appreciated. The authors are also grateful to William Shanks, Vincent Arena, Thomas McConkey, and Serafino Carri for important technical support. The authors thank the group of David Pappas at NIST for fabricating the directional coupler chip and its package. Work pertaining to the development of the Purcell filter was supported by IARPA under contract W911NF10-1-0324 and work pertaining to the development of the pogo-pin packaging was supported by IARPA under contract W911NF-16-1-0114-FE. This article is a contribution of the U.S. Government, not subject to copyright.

\section{APPENDIX A: MPIJIS SCATTERING PARAMETERS}

To derive analytical expressions for the scattering parameters of the MPIJIS, we solve the effective signal flow graph of the device shown in Fig. 10(a), which includes the coupled JPCs operated in frequencyconversion mode. On-resonance signals at $f_{1}=f_{a}$ or $f_{2}=$ $f_{b}$ input at port a (e.g., $1^{\prime}$ or $2^{\prime}$ ) or port b (e.g., b1 or b2) are reflected with reflection parameter $r$ and transmitted with frequency conversion to the other port with transmission parameter $t$, where $r$ and $t$ are determined by the pump drive amplitude and satisfy the energy-conservation condition $r^{2}+t^{2}=1$. More specifically, in the stiff pump 
(a)
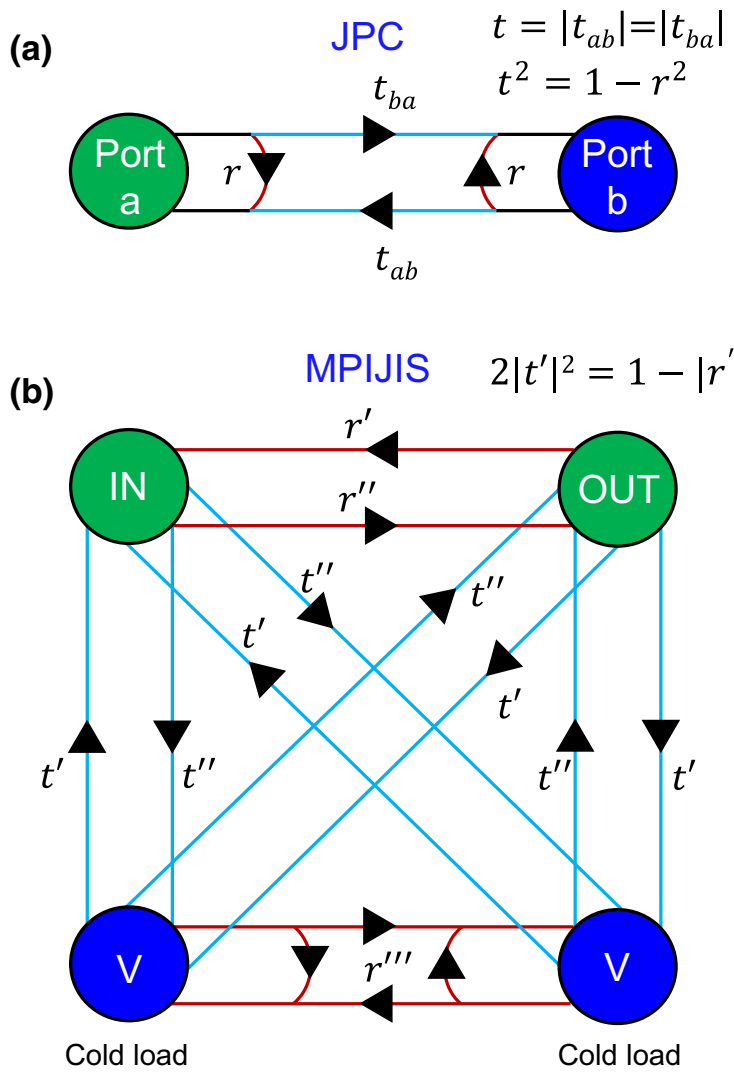

FIG. 11. Simplified signal flow graph for a JPC operated in frequency-conversion mode (a) and for the MPIJIS (b). See Appendix $\mathrm{C}$ for details.

approximation, $r$ and $t$ can be written as $[38,43]$

$$
\begin{aligned}
& r=\frac{1-\rho^{2}}{1+\rho^{2}}, \\
& t=\frac{2 \rho}{1+\rho^{2}},
\end{aligned}
$$

where $0 \leq \rho$ is a dimensionless pump amplitude. For $\rho=$ 0 , the JPC acts as a perfect mirror, whereas for $\rho=1$, the JPC operates in full frequency-conversion mode between ports $a$ and $b$.

In the derivation, we assume that the two JPCs are balanced (i.e., their reflection and transmission parameters are equal). Figure 10(a) also includes flow graphs of two couplers coupling the $\mathrm{a}$ and $\mathrm{b}$ ports of the JPCs; one represents the $90^{\circ}$ hybrid, which couples between the a ports of the JPCs, while the other is fictitious, coupling the $b$ ports. The latter models the amplitude attenuation $\alpha$ present on the $\mathrm{b}$ port due to signal absorption in the $50-\Omega$ cold loads. Because of the structural symmetry of the device, we consider a coupler with real coefficients $\alpha$ and $\beta$, which satisfy the condition $\alpha^{2}+\beta^{2}=1$. For an ideal symmetric coupler (i.e., $90^{\circ}$ hybrid), $\alpha=\beta=1 / \sqrt{2}$ [19].

A detailed derivation of the scattering parameters of the MPIJIS is given in the supplementary information in Ref.
[35]. For completeness, we provide in this section the main results of Ref. [35] and update the equations to account for the effect of the applied fluxes in the JRMs on the isolation direction of the MPIJIS. More specifically, we generalize the nonreciprocal phases $\phi_{p 1}$ and $\phi_{p 2}$ acquired by the frequency-converted transmitted signals between ports a and b, which in Ref. [35] represent only the phases of the pump drives feeding JPC 1 and JPC 2 at frequency $f_{p}$, to $\phi_{p 1}^{\prime}=\phi_{p 1}+i n_{g 1} \pi$ and $\phi_{p 2}^{\prime}=\phi_{p 2}+i n_{g 2} \pi$, which account for the sign of the real coupling constant $g_{a b}$ (i.e., +1 and -1 obtained for $n_{g}=0$ and $n_{g}=1$, respectively) set by the sign of the applied flux in the JRM or alternatively the direction of the circulating current [64]. Consequently, the multiplication of the transmission parameters through the two JPCs results in multiplication of the signs of the coupling constants or addition of the corresponding phases [i.e., $e^{i p \pi}$, where $\left.p=\left(n_{g 1}+n_{g 2}\right) \bmod 2\right]$. After introducing this update and expressing the MPIJIS scattering parameters in terms of the parameter $t$, we obtain on resonance

$$
\begin{aligned}
& S_{21}=\frac{i}{1+\frac{\alpha^{2}}{\beta^{2}} t^{2}}\left(\sqrt{1-t^{2}}-\frac{\alpha}{\beta^{2}} t^{2} \sin \varphi\right), \\
& S_{12}=\frac{i}{1+\frac{\alpha^{2}}{\beta^{2}} t^{2}}\left(\sqrt{1-t^{2}}+\frac{\alpha}{\beta^{2}} t^{2} \sin \varphi\right), \\
& S_{11}=S_{22}=-\frac{i \alpha}{\beta^{2}} \frac{t^{2}}{1+\frac{\alpha^{2}}{\beta^{2}} t^{2}} \cos \varphi,
\end{aligned}
$$

$$
\begin{aligned}
& S_{33}=S_{44}=-\frac{\sqrt{1-t^{2}}}{1+\frac{\alpha^{2}}{\beta^{2}} t^{2}}, \\
& S_{34}=S_{43}=\frac{\alpha}{\beta^{2}} \frac{t^{2}}{1+\frac{\alpha^{2}}{\beta^{2}} t^{2}},
\end{aligned}
$$

$$
S_{13}=-\frac{t e^{-i \varphi_{S} / 2+i \pi / 4}}{\sqrt{2} \beta\left(1+\frac{\alpha^{2}}{\beta^{2}} t^{2}\right)}\left(\sqrt{1-t^{2}} \alpha e^{i \frac{\varphi}{2}+i \frac{\pi}{4}}+e^{-i \frac{\varphi}{2}-i \frac{\pi}{4}}\right) \text {, }
$$

$$
S_{14}=-\frac{t e^{-i \varphi_{s} / 2+i \pi / 4}}{\sqrt{2} \beta\left(1+\frac{\alpha^{2}}{\beta^{2}} t^{2}\right)}\left(e^{i \frac{\varphi}{2}+i \frac{\pi}{4}}+\sqrt{1-t^{2}} \alpha e^{-i \frac{\varphi}{2}-i \frac{\pi}{4}}\right),
$$

$$
\begin{aligned}
& S_{23}=-\frac{t e^{-i \varphi_{s} / 2+i \pi / 4}}{\sqrt{2} \beta\left(1+\frac{\alpha^{2}}{\beta^{2}} t^{2}\right)}\left(\sqrt{1-t^{2}} \alpha e^{i \frac{\varphi}{2}-i \frac{\pi}{4}}+e^{-i \frac{\varphi}{2}+i \frac{\pi}{4}}\right), \\
& S_{24}=-\frac{t e^{-i \varphi_{s} / 2+i \pi / 4}}{\sqrt{2} \beta\left(1+\frac{\alpha^{2}}{\beta^{2}} t^{2}\right)}\left(e^{i \frac{\varphi}{2}-i \frac{\pi}{4}}+\sqrt{1-t^{2}} \alpha e^{-i \frac{\varphi}{2}+i \frac{\pi}{4}}\right),
\end{aligned}
$$




$$
\begin{aligned}
& S_{31}=-\frac{t e^{i \varphi_{s} / 2+i \pi / 4}}{\sqrt{2} \beta\left(1+\frac{\alpha^{2}}{\beta^{2}} t^{2}\right)}\left(\sqrt{1-t^{2}} \alpha e^{-i \frac{\varphi}{2}+i \frac{\pi}{4}}+e^{i \frac{\varphi}{2}-i \frac{\pi}{4}}\right), \\
& S_{32}=-\frac{t e^{i \varphi_{s} / 2+i \pi / 4}}{\sqrt{2} \beta\left(1+\frac{\alpha^{2}}{\beta^{2}} t^{2}\right)}\left(\sqrt{1-t^{2}} \alpha e^{-i \frac{\varphi}{2}-i \frac{\pi}{4}}+e^{i \frac{\varphi}{2}+i \frac{\pi}{4}}\right), \\
& S_{41}=-\frac{t e^{i \varphi_{s} / 2+i \pi / 4}}{\sqrt{2} \beta\left(1+\frac{\alpha^{2}}{\beta^{2}} t^{2}\right)}\left(e^{-i \frac{\varphi}{2}+i \frac{\pi}{4}}+\sqrt{1-t^{2}} \alpha e^{i \frac{\varphi}{2}-i \frac{\pi}{4}}\right), \\
& S_{42}=-\frac{t e^{i \varphi_{s} / 2+i \pi / 4}}{\sqrt{2} \beta\left(1+\frac{\alpha^{2}}{\beta^{2}} t^{2}\right)}\left(e^{-i \frac{\varphi}{2}-i \frac{\pi}{4}}+\sqrt{1-t^{2}} \alpha e^{i \frac{\varphi}{2}+i \frac{\pi}{4}}\right) .
\end{aligned}
$$

While Eqs. (A2)-(A14) have the same form as those derived in Ref. [35], the phases $\varphi$ and $\varphi_{s}$ are different. In this case, they are given by $\varphi \equiv \varphi_{p}+p \pi$ and $\varphi_{s} \equiv$ $\phi_{p 1}+\phi_{p 2}+p \pi$, where $\varphi_{p}=\phi_{p 1}-\phi_{p 2}$.

Before we outline the effect of the parity parameter $p$ on the MPIJIS response, we consider two important cases:

(1) No applied pump, i.e., $t=0$. In this case, the scattering matrix reduces to

$$
[S]=\left(\begin{array}{cccc}
0 & i & 0 & 0 \\
i & 0 & 0 & 0 \\
0 & 0 & -1 & 0 \\
0 & 0 & 0 & -1
\end{array}\right)
$$

This result shows that when the MPIJIS is off, it is transparent for propagating signals and effectively behaves as a lossless transmission line with an added reciprocal phase shift of $\pi / 2$ for transmitted signals within the bandwidth of the $90^{\circ}$ hybrid.

(2) The JPCs are biased at the working point of the 50:50 beam splitter, i.e., $r=t=1 / \sqrt{2}$, the b-mode coupler is symmetrical, $\alpha=\beta=1 / \sqrt{2}$, the phase difference is $\varphi=-\pi / 2$, and the sum is $\varphi_{s}=\pi / 2$. In this case, the scattering matrix becomes

$$
[S]=\left(\begin{array}{cccc}
0 & 0 & -\frac{1}{\sqrt{2}} & -\frac{1}{\sqrt{2}} \\
\frac{i 2 \sqrt{2}}{3} & 0 & -\frac{i}{3 \sqrt{2}} & \frac{i}{3 \sqrt{2}} \\
-\frac{1}{3 \sqrt{2}} & -\frac{i}{\sqrt{2}} & -\frac{\sqrt{2}}{3} & \frac{\sqrt{2}}{3} \\
\frac{1}{3 \sqrt{2}} & -\frac{i}{\sqrt{2}} & \frac{\sqrt{2}}{3} & -\frac{\sqrt{2}}{3}
\end{array}\right),
$$

which shows that, under the above conditions, the MPIJIS functions as an isolator with almost unity transmission in the forward direction, i.e., $\left|S_{21}\right|=$ $2 \sqrt{2} / 3 \cong 0.943$ (which corresponds to an insertion loss of about $0.5 \mathrm{~dB}$ in the signal power), total isolation in the opposite direction, $\left|S_{12}\right|=0$, and vanishing reflections, $\left|S_{11}\right|=\left|S_{22}\right|=0$. Furthermore, it shows that the cold loads on ports 3 and 4 play a similar role to internal ports of standard magnetic isolators. They dissipate the energy of backpropagating signals, $\left|S_{32}\right|=\left|S_{42}\right|=1 / \sqrt{2}$, and emit noise (e.g., vacuum noise) towards the input, $\left|S_{13}\right|=$ $\left|S_{14}\right|=1 / \sqrt{2}$.

Although Eqs. (A2)-(A14) are derived for on-resonance signals, it is straightforward to generalize them for signals that lie within the JPC dynamical bandwidth. This is done by substituting [38]

$$
t\left[\omega_{1}\right]=\frac{2 \rho}{\chi_{a}^{-1} \chi_{b}^{-1}+\rho^{2}},
$$

where $\chi_{a}$ and $\chi_{b}$ are the bare response functions of modes $\mathrm{a}$ and $\mathrm{b}$ (whose inverses depend linearly on $f_{1}$ and $f_{2}$ ):

$$
\begin{aligned}
& \chi_{a}^{-1}\left[\omega_{1}\right]=1-2 i \frac{\omega_{1}-\omega_{a}}{\gamma_{a}}, \\
& \chi_{b}^{-1}\left[\omega_{1}\right]=1-2 i \frac{\omega_{1}-\omega_{a}}{\gamma_{b}},
\end{aligned}
$$

where $\omega_{1}=2 \pi f_{1}, \omega_{a}=2 \pi f_{a}$, and the applied pump angular frequency is given by $\omega_{p}=\omega_{b}-\omega_{a}=\omega_{2}-\omega_{1}$, where $\omega_{b}=2 \pi f_{b}$ and $\omega_{2}=2 \pi f_{2}$.

This generalization holds under the assumption that the bandwidth of the $90^{\circ}$ hybrid is much larger than the 3-12-MHz dynamical bandwidths of the JPCs, which is generally the case because transmission-line-based hybrids typically exhibit bandwidths of a few hundred megahertz [65].

\section{APPENDIX B: EFFECTIVE TWO-PORT MODEL}

Here we derive an effective two-port model of the MPIJIS, which calculates the scattering parameters of ports 1 and 2 only. In this model, we replace the effective coupler shown in Fig. 10(a) by a lossy delay line, coupling mode b of the two JPCs as depicted in Fig. 10(b). The transmission coefficient of this delay line is frequency dependent and can be expressed as $\alpha\left[\omega_{1}\right]=|\alpha| e^{i \theta_{d}}$, where the phase delay reads $\theta_{d}\left[\omega_{1}\right]=\omega_{2} \sqrt{\epsilon_{\text {effl }}} l_{d} / c=\left(\omega_{1}+\omega_{p}\right) \sqrt{\epsilon_{\text {effl }}} l_{d} / c$, where $l_{d}=11.283 \mathrm{~mm}$ is the length of the microstrip transmission line coupling the two JPCs, $c$ is the speed of light, and $\epsilon_{\text {eff }}=7.418$ is the effective dielectric constant of the microstrip transmission line. The amplitude $0 \leq|\alpha| \leq 1$ represents the amplitude attenuation of the internal mode $\mathrm{b}$ due to coupling to the cold terminations.

To calculate the device scattering parameters at ports 1 and 2, we start off by writing the scattering parameters of the inner device defined by ports $1^{\prime}$ and $2^{\prime}$, which excludes 
the $90^{\circ}$ hybrid. Using the signal flow graph shown in Fig. 10(b), we obtain

$$
\begin{aligned}
& s_{1^{\prime} 2^{\prime}}\left[\omega_{1}\right]=-\frac{\alpha t^{2} e^{-i \varphi}}{1-r_{b}^{2} \alpha^{2}}, \\
& s_{2^{\prime} 1^{\prime}}\left[\omega_{1}\right]=-\frac{\alpha t^{2} e^{i \varphi}}{1-r_{b}^{2} \alpha^{2}}, \\
& s_{1^{\prime} 1^{\prime}}\left[\omega_{1}\right]=s_{2^{\prime} 2^{\prime}}\left[\omega_{1}\right]=r_{a}-\frac{r_{b} \alpha^{2} t^{2}}{1-r_{b}^{2} \alpha^{2}},
\end{aligned}
$$

where the JPC transmission coefficient is given by Eq. (A17) and $r_{a}$ and $r_{b}$ are the JPC reflection parameters at ports $\mathrm{a}$ and $\mathrm{b}$ given by

$$
\begin{aligned}
& r_{a}\left[\omega_{1}\right]=\frac{\chi_{a}^{-1 *} \chi_{b}^{-1}-\rho^{2}}{\chi_{a}^{-1} \chi_{b}^{-1}+\rho^{2}}, \\
& r_{b}\left[\omega_{1}\right]=\frac{\chi_{a}^{-1} \chi_{b}^{-1 *}-\rho^{2}}{\chi_{a}^{-1} \chi_{b}^{-1}+\rho^{2}} .
\end{aligned}
$$

The inverse bare response functions $\chi_{a}^{-1}$ and $\chi_{b}^{-1}$ of modes $\mathrm{a}$ and $\mathrm{b}$ are given by Eq. (A18).

After including the wave interference effect introduced by the $90^{\circ}$ hybrid, we finally arrive at the following expressions for the scattering matrix of the device:

$$
\begin{aligned}
& S_{21}\left[\omega_{1}\right]=\frac{1}{2}\left(i s_{1^{\prime} 1^{\prime}}+i s_{2^{\prime} 2^{\prime}}+s_{2^{\prime} 1^{\prime}}-s_{1^{\prime} 2^{\prime}}\right), \\
& S_{12}\left[\omega_{1}\right]=\frac{1}{2}\left(i s_{1^{\prime} 1^{\prime}}+i s_{2^{\prime} 2^{\prime}}+s_{1^{\prime} 2^{\prime}}-s_{2^{\prime} 1^{\prime}}\right), \\
& S_{11}\left[\omega_{1}\right]=\frac{1}{2}\left(s_{1^{\prime} 1^{\prime}}-s_{2^{\prime} 2^{\prime}}+i s_{2^{\prime} 1^{\prime}}+i s_{1^{\prime} 2^{\prime}}\right), \\
& S_{22}\left[\omega_{1}\right]=\frac{1}{2}\left(s_{2^{\prime} 2^{\prime}}-s_{1^{\prime} 1^{\prime}}+i s_{2^{\prime} 1^{\prime}}+i s_{1^{\prime} 2^{\prime}}\right) .
\end{aligned}
$$

To calculate the MPIJIS transmission response versus frequency that matches the measured curves in Fig. 3, we evaluate $\left|S_{21}\right|^{2}$ and $\left|S_{12}\right|^{2}$ on the basis of Eqs. (B6) and (B7), while substituting $\omega_{p} / 2 \pi=2.727 \mathrm{GHz}$ (applied in the experiment) and $\varphi=-\pi / 2(\varphi=\pi / 2)$ when $P_{1}\left(P_{2}\right)$ is driven. We also substitute $\gamma_{a} / 2 \pi=40 \mathrm{MHz}$ (measured), $\gamma_{b} / 2 \pi=100 \mathrm{MHz}$ (set to match the bandwidths of the measured curves), and $|\alpha|=0.51$. The latter parameter (i.e., $|\alpha|$ ) is evaluated by our varying $\rho$ and $|\alpha|$ to simultaneously best match one pair of $\left|S_{21}\right|^{2}$ and $\left|S_{12}\right|^{2}$ values measured on resonance for the same pump power and pump port. This is because, on resonance, the device response in both directions is dependent solely on these two parameters. Next, for each pair of curves $\left|S_{21}\right|^{2}$ and $\left|S_{12}\right|^{2}$ measured for the same pump power and pump port, we substitute $\omega_{a} / 2 \pi$ corresponding to the frequency of the respective isolation dip. This is done because the theoretical model here does not account for shifts in the
JPC resonance frequency due to the Kerr effect. Finally, for each pair of curves $\left(\left|S_{21}\right|^{2}\right.$ and $\left|S_{12}\right|^{2}$ corresponding to the same pump power and pump port), we substitute $\rho$, which gives the respective isolation dip magnitude on resonance.

One likely physical reason for the deviation of the parameter value $|\alpha|=0.51$ in our device from $1 / \sqrt{2}$ expected for an ideal symmetric coupler [in which $\alpha$, the coupling coefficient between the $\mathrm{b}$ modes of the two JPCs, is equal to $\beta$, the coupling coefficient of each JPC to the cold termination; see Fig. 10(a)] is the presence of two coupling capacitors in series between the two JPCs, which couple the JPCs to the intermediate delay line [see the device configuration shown in Figs. 1(d) and 1(g)]. Having these two coupling capacitors in series in the path between the two JPCs, instead of one, effectively reduces the coupling for wave amplitudes by $\sqrt{2}$, thus giving $|\alpha|=0.5$, which matches well the estimated value of 0.51 in our device.

\section{APPENDIX C: COMMON ATTRIBUTES OF THE MPIJIS AND JPC}

To better understand the scattering parameters of the MPIJIS, we outline in Figs. 11(a) and 11(b) the onresonance signal flow graph for a JPC operated in frequency-conversion mode and a MPIJIS operated in the forward direction, respectively. If we suppress the phases of the various scattering parameters between the ports and focus on their magnitude, then the JPC can be characterized by two parameters, a reflection parameter $r$ and a transmission parameter $t$ given by Eq. (A1), which satisfy the condition $t^{2}=1-r^{2}$. Similarly, in the MPIJIS case, we can reduce its various scattering parameters between the input port (1), output port (2), and vacuum ports (3 and 4) given by Eqs. (A2)-(A14) into five main parameters, denoted as $r^{\prime}, r^{\prime \prime}, r^{\prime \prime \prime}, t^{\prime}$, and $t^{\prime \prime}$ in Fig. 11(b). Assuming the two JPCs in the MPIJIS are uniform and balanced, we express these parameters as

$$
\begin{aligned}
r^{\prime} & =\frac{r-\alpha}{1-\alpha r}, \\
r^{\prime \prime} & =\frac{r+\alpha}{1+\alpha r}, \\
r^{\prime \prime \prime} & =\frac{r}{1+\frac{\alpha^{2}}{1-\alpha^{2}} t^{2}}, \\
t^{\prime} & =\frac{1}{\sqrt{2}} \frac{\sqrt{1-\alpha^{2}}}{1-\alpha r} t, \\
t^{\prime \prime} & =\frac{1}{\sqrt{2}} \frac{\sqrt{1-\alpha^{2}}}{1+\alpha r} t,
\end{aligned}
$$

where $r$ and $t$ are the scattering parameters of one JPC stage and $\alpha$ is the amplitude attenuation of the internal mode. 


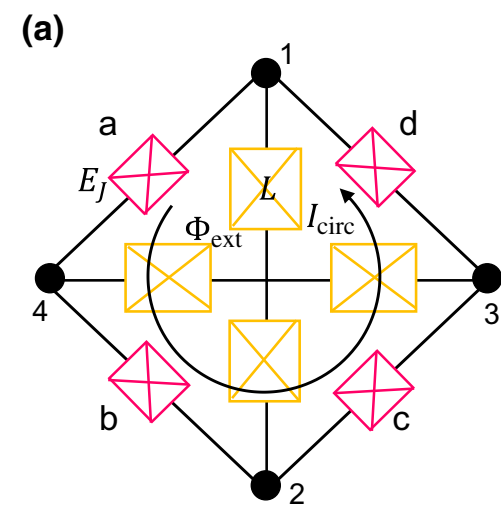

(b)

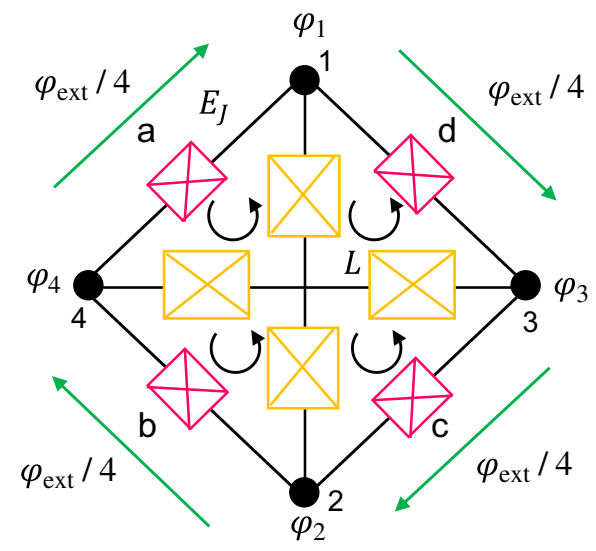

(c)

Differential mode

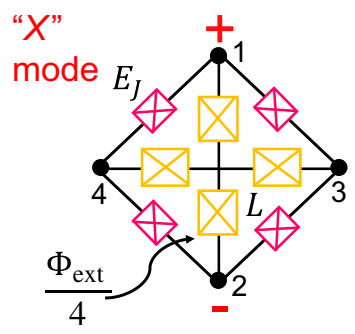

(d)

Differential mode

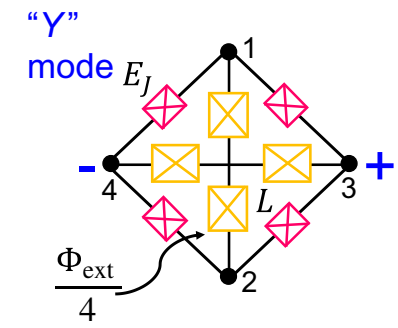

(g)

(f)

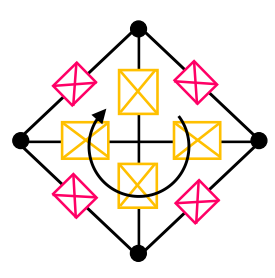

$\varphi_{\text {ext }}<0$ (e)

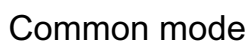

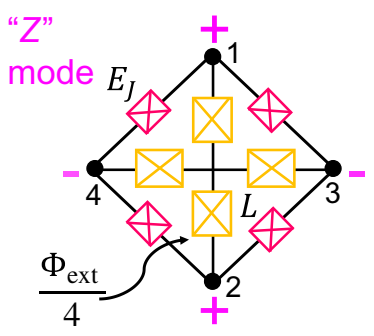

(h)

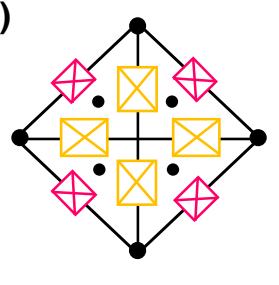

$\varphi_{\text {ext }}=0$
FIG. 12. JRM structure, eigenmodes, and circulating currents. (a) The JRM consists of four Josephson junctions a, b, c, and d with energy $E_{J}$, which are arranged in a Wheatstone bridge configuration between nodes $1,2,3$, and 4 . The four large inner junctions, each with inductance $L$, function as linear shunt inductors for the JRM junctions. An externally applied magnetic flux threading the JRM loop gives rise to a circulating direct current $I_{\text {circ }}$. (b) The reduced node fluxes $\varphi_{1}, \varphi_{2}$, $\varphi_{3}$, and $\varphi_{4}$ used in the calculation of the JRM eigenmodes. The four inner loops are assumed to be equal in size, and thus each loop receives a quarter of $\Phi_{\text {ext }}$. When the JRM is biased at a primary flux lobe, no direct current flows in the shunt inductances inside the JRM (i.e., the circulating currents in the inner branches cancel each other). (c)-(e) Polarity of the rf node voltages corresponding to the eigenmodes of the JRM. (c),(d) Polarity patterns for the differential excitation modes " $X$ " and "Y," respectively. (e) The pattern for the common excitation mode " $Z$." (f)-(h) Correspondence between the circulating current direction and the sign of the reduced external magnetic flux $\varphi_{\text {ext }}$. The black circles in $(\mathrm{g})$ indicate no circulating currents.
In the special case where $r=t=\alpha=1 / \sqrt{2}$, we retrieve the result of Eq. (A16). It is also straightforward to see that in the limit $r \rightarrow \alpha$, we get $r^{\prime} \rightarrow 0, r^{\prime \prime} \gg r^{\prime}$, and $t^{\prime} \gg t^{\prime \prime}$.

Interestingly, the first three parameters $\left(r^{\prime}, r^{\prime \prime}\right.$, and $\left.r^{\prime \prime \prime}\right)$ exhibit a reflectionlike dependence on $\rho$ despite representing transmission parameters between ports, in particular $r^{\prime}$ and $r^{\prime \prime}$, which are the MPIJIS transmission in the backward direction and the forward direction, respectively. This can be seen, for example, by substituting Eq. (A1) into Eqs. (C1) and (C2), which gives

$$
\begin{aligned}
& r^{\prime}=\frac{1-\alpha^{\prime} \rho^{2}}{1+\alpha^{\prime} \rho^{2}} \\
& r^{\prime \prime}=\frac{1-\alpha^{\prime \prime} \rho^{2}}{1+\alpha^{\prime \prime} \rho^{2}}
\end{aligned}
$$

where $\alpha^{\prime}=(1+\alpha) /(1-\alpha)$ and $\alpha^{\prime \prime}=1 / \alpha^{\prime}$. Casting $r^{\prime}$ and $r^{\prime \prime}$ in this form implies that they generally behave as

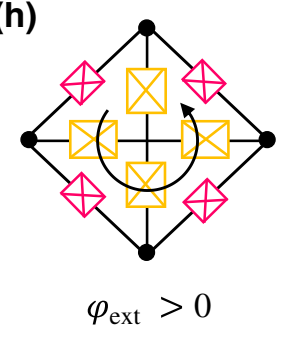

$r$ of a JPC [see Eq. (A1)] with rescaled pump amplitudes. Since $\alpha^{\prime} \geq 1\left(\alpha^{\prime \prime} \leq 1\right)$, we find that in the limit $\rho^{2} \rightarrow \alpha^{\prime \prime}$, we get $r^{\prime} \approx 0$ and $r^{\prime \prime} \approx\left(1-\alpha^{\prime \prime 2}\right) /\left(1+\alpha^{\prime \prime 2}\right)$.

Extending this observation to the scattering parameters of the MPIJIS and JPC, we find that they generally exhibit a reflectionlike dependence on $\rho$ (indicated by red lines in Fig. 11) if they link between the same mode a or b regardless of their physical ports and a transmissionlike dependence if they link between different modes (indicated by cyan lines in Fig. 11).

Furthermore, by inspecting the signal flow graph in Fig. 11(b), we derive a useful energy-conservation condition for the MPIJIS:

$$
T \equiv 2\left|t^{\prime}\right|^{2}=1-\left|r^{\prime}\right|^{2}=\frac{t^{2}}{1-\alpha^{2}},
$$

which states that the energy of the signal entering through the output port of the MPIJIS is either dissipated in the loads or passed to the input. To a large extent, this is 
analogous to the condition of a JPC operated in frequencyconversion mode; namely, $T \equiv t^{2}=1-r^{2}$.

Equation (C8) is important for two reasons: (1) It shows that for a signal entering the output, the MPIJIS with $t \rightarrow$ $1-\alpha^{2}$ mimics a JPC with $t \rightarrow 1$ (operated in full conversion mode). But unlike the JPC, in which a small portion of the signal is reflected back to the same port, in the MPIJIS case, it is transmitted to a different port, (i.e., the input). (2) It allows us to infer the amount of energy of the signal coming from the output that gets dissipated in the loads (i.e., $2\left|t^{\prime}\right|^{2}$ ) by measuring the isolation of the MPIJIS (i.e., $\left.\left|r^{\prime}\right|^{2}\right)$.

\section{APPENDIX D: MPIJIS SATURATION DUE TO PUMP DEPLETION}

To evaluate the saturation of the MPIJIS due to pump depletion effects, we rely on the equivalence, established in Appendix $\mathrm{C}$, between processing of incoming signals through the output port of a MPIJIS and operating a JPC in frequency-conversion mode. We also apply, in our calculation, the same method and definitions as in Ref. [38] used for evaluating the saturation of a JPC due to pump depletion effects in the amplification mode.

We start off with the quantum Langevin equation for the pump mode $c$ of a JPC operated in conversion mode, which reads

$$
\frac{d}{d t} c=-i \omega_{c} c-i g_{3} a^{\dagger} b-\frac{\gamma_{c}}{2} c+\sqrt{\gamma_{c}} c^{i n}(t),
$$

where the input field is given by

$$
\tilde{c}^{\text {in }}(t)=\frac{1}{\sqrt{2 \pi}} \int_{0}^{\infty} c^{\text {in }}[\omega] e^{-i \omega t} d \omega
$$

and satisfies the commutation relation $\left[c^{\mathrm{in}}[\omega], c^{\mathrm{in}}\left[\omega^{\prime}\right]\right]=$ $\operatorname{sgn}\left(\left(\omega-\omega^{\prime}\right) / 2\right) \delta\left(\omega+\omega^{\prime}\right)$.

Taking the average value for the field $c$ gives

$$
\frac{d}{d t}\langle c\rangle=-i \omega_{c}\langle c\rangle-i g_{3}\left\langle a^{\dagger} b\right\rangle-\frac{\gamma_{c}}{2}\langle c\rangle+\sqrt{\gamma_{c}}\left\langle\tilde{c}^{\text {in }}(t)\right\rangle .
$$

In the steady state and using the rotating-waveapproximation, we obtain

$$
i g_{3}\left\langle a^{\dagger} b\right\rangle+\frac{\gamma_{c}}{2}\langle c\rangle=\sqrt{\gamma_{c}}\left\langle\tilde{c}^{\text {in }}(t)\right\rangle
$$

In the limit of no input $\left(\left\langle a^{\dagger} b\right\rangle=0\right)$ we get

$$
\langle c\rangle=\frac{2}{\sqrt{\gamma_{c}}}\left\langle\tilde{c}^{\text {in }}(t)\right\rangle .
$$

In this case the average number of photons in the resonator $c$ is

$$
\lim _{\left\langle a^{\dagger} b\right\rangle \rightarrow 0} \bar{n}_{c}=|\langle c\rangle|^{2}=\frac{4}{\gamma_{c}}\left|\left\langle\tilde{c}^{\text {in }}(t)\right\rangle\right|^{2}=\frac{4}{\gamma_{c}} \bar{n}_{c}^{\text {in }},
$$

where $\bar{n}_{c}^{\text {in }}$ is the average number of input pump photons per unit time.

In the presence of input $\left\langle a^{\dagger} b\right\rangle \neq 0$, the pump drive experiences an additional decay channel associated with the photon-conversion process occurring in the device. Thus, we define an effective decay rate of pump photons $\gamma_{\text {eff }}$ given by

$$
i g_{3}\left\langle a(t)^{\dagger} b(t)\right\rangle=\frac{\gamma_{\mathrm{eff}}}{2}\langle c(t)\rangle .
$$

To calculate $\gamma_{\text {eff }}$, we first evaluate $\left\langle a(t)^{\dagger} b(t)\right\rangle$ in the frame rotating with the pump phase:

$$
\left\langle a(t)^{\dagger} b(t)\right\rangle=\frac{1}{2 \pi} \int_{-\infty}^{\infty} \int_{-\infty}^{\infty}\left\langle a[\omega] b\left[\omega^{\prime}\right]\right\rangle e^{-i\left(\omega+\omega^{\prime}\right) t} d \omega d \omega^{\prime} .
$$

Using the JPC scattering parameters in conversion mode [38], and the input-output relations given by

$$
\sqrt{\gamma_{a}} a[\omega]=a^{\text {in }}[\omega]+a^{\text {out }}[\omega],
$$

$$
\sqrt{\gamma_{b}} b\left[\omega^{\prime}\right]=b^{\text {in }}\left[\omega^{\prime}\right]+b^{\text {out }}\left[\omega^{\prime}\right]
$$

we obtain

$$
\begin{aligned}
\left\langle a[\omega] b\left[\omega^{\prime}\right]\right\rangle= & \frac{-i T(\Delta \omega)}{\sqrt{\gamma_{a} \gamma_{b}} \rho}\left[\left\langle a^{\text {in }}[\omega] a^{\text {in }}\left[\omega^{\prime}\right]\right\rangle\right. \\
& \left.+\left\langle b^{\text {in }}[\omega] b^{\text {in }}\left[\omega^{\prime}\right]\right\rangle\right],
\end{aligned}
$$

where $T(\Delta \omega)=|t|^{2}=4 \rho^{2} /\left|\chi_{a}^{-1} \chi_{b}^{-1}+\rho^{2}\right|^{2}$.

Substituting the anticommutator for the noise field given by

$$
\begin{aligned}
\left\langle\left\{a^{\text {in }}[\omega], a^{\text {in }}\left[\omega^{\prime}\right]\right\}\right\rangle_{T} & =\left\langle\left\{b^{\text {in }}[\omega], b^{\text {in }}\left[\omega^{\prime}\right]\right\}\right\rangle_{T} \\
& =2 \mathcal{N}_{T}\left(\frac{\omega-\omega^{\prime}}{2}\right) \delta\left(\omega+\omega^{\prime}\right)
\end{aligned}
$$

into Eqs. (D11) and (D8) yields

$$
\begin{aligned}
\left\langle a(t)^{\dagger} b(t)\right\rangle= & \frac{-2 i}{\sqrt{\gamma_{a} \gamma_{b}} \rho} \frac{1}{2 \pi} \int_{0}^{\infty} \\
& \times\left[\mathcal{N}_{a}^{\text {in }}(\omega)+\mathcal{N}_{b}^{\text {in }}(\omega)\right] T(\Delta \omega) d \omega,
\end{aligned}
$$


where $\mathcal{N}_{T}$ is the photon spectral density given by

$$
\begin{aligned}
\mathcal{N}_{T}[\omega] & =\frac{\operatorname{sgn} \omega}{2} \operatorname{coth}\left(\frac{\hbar \omega}{2 k_{B} T}\right) \\
& =\operatorname{sgn} \omega\left(N_{T}[|\omega|]+\frac{1}{2}\right)
\end{aligned}
$$

and $N_{T}[\omega]=1 /\left(e^{\hbar \omega / k_{B} T}-1\right)$ is the Bose-Einstein distribution.

Ignoring vacuum noise, which is much smaller than the thermal noise coming from the $4 \mathrm{~K}$ stage and couples to mode a, we obtain

$$
\left\langle a(t)^{\dagger} b(t)\right\rangle=\frac{-2 i}{\sqrt{\gamma_{a} \gamma_{b}} \rho} \frac{1}{2 \pi} \int_{0}^{\infty} N_{a}^{\mathrm{in}}(\omega)[1-L(\omega)] d \omega,
$$

where we applied Eq. (C8), which links the transmission of a JPC, $T(\Delta \omega)$, to the reverse transmission of a MPIJIS device, $L(\omega)$.

Substituting Eq. (D14) in Eq. (D7) along with Eq. (D6) and the relation $\rho=2 g_{3} \sqrt{\bar{n}_{c}} / \sqrt{\gamma_{a} \gamma_{b}}$, we obtain the simple relation

$$
\gamma_{\mathrm{eff}}=\gamma_{c} \frac{\bar{n}_{n}^{\mathrm{in}}}{4 \bar{n}_{c}^{\mathrm{in}}}
$$

where $\bar{n}_{n}^{\text {in }} \equiv \frac{1}{\pi} \int_{0}^{\infty} N_{a}^{\text {in }}(\omega)[1-L(\omega)] d \omega$ represents the average number of input noise photons per unit time that are transferred to the MPIJIS loads.

Next, we note from Eq. (D6) that for a given set point $L_{0}$, the average number of pump photons in the device with no applied noise is given by

$$
\bar{n}_{c}\left(L_{0}, \bar{n}_{n}^{\mathrm{in}}=0\right)=\frac{4}{\gamma_{c}} \bar{n}_{c}^{\mathrm{in}},
$$

whereas in the presence of noise or input signal it is given by

$$
\bar{n}_{c}\left(L_{0}, \bar{n}_{n}^{\mathrm{in}}\right)=\frac{4 \gamma_{c}}{\left(\gamma_{c}+\gamma_{\mathrm{eff}}\left(L_{0}\right)\right)^{2}} \bar{n}_{c}^{\mathrm{in}}
$$

Thus, for a fixed input pump power (i.e., fixed $\bar{n}_{c}^{\mathrm{in}}$ ) and using Eqs. (D15)-(D17), we get

$$
\begin{aligned}
\frac{\bar{n}_{c}\left(L_{0}, \bar{n}_{n}^{\text {in }}\right)}{\bar{n}_{c}\left(L_{0}, \bar{n}_{n}^{\text {in }}=0\right)} & =\frac{1}{\left(1+\frac{\bar{n}_{n}^{\text {in }}\left(L_{0}\right)}{4 \bar{n}_{c}^{\text {in }}}\right)^{2}} \\
& \cong\left(1-\frac{\bar{n}_{n}^{\text {in }}\left(L_{0}\right)}{2 \bar{n}_{c}^{\text {in }}}\right) .
\end{aligned}
$$

On the other hand, on resonance we have

$$
\frac{\bar{n}_{c}\left(L_{0}, \bar{n}_{n}^{\text {in }}\right)}{\bar{n}_{c}\left(L_{0}, \bar{n}_{n}^{\text {in }}=0\right)}=\frac{\frac{1-\sqrt{L}}{1+\sqrt{L}}}{\frac{1-\sqrt{L_{0}}}{1+\sqrt{L_{0}}}},
$$

where we used the relation $\rho^{2}=\frac{1}{\alpha^{\prime}} \frac{1-\sqrt{L}}{1+\sqrt{L}}$ derived from Eq. (C6) (in which $r^{\prime} \equiv \sqrt{L}$ ) and the dependence of $\rho$ on $\bar{n}_{c}$.

Combining the results of Eqs. (D18) and (D19) gives for $L \ll 1$

$$
2 \sqrt{L_{0}}\left(\sqrt{\frac{L}{L_{0}}}-1\right)=\frac{\bar{n}_{n}^{\text {in }}\left(L_{0}\right)}{2 \bar{n}_{c}^{\text {in }}} .
$$

Hence, if we limit the deviation of $L$ compared with $L_{0}$ to $L / L_{0}<1+\epsilon$, where $\epsilon \ll 1$, then the device saturation due to pump depletion can be considered small if the ratio of the input noise photons to input pump photons satisfies the inequality

$$
\frac{\bar{n}_{n}^{\text {in }}\left(L_{0}\right)}{2 \bar{n}_{c}^{\text {in }}}<\epsilon \sqrt{L_{0}} .
$$

\section{APPENDIX E: THE EFFECT OF PUMP DEPLETION ON THE DEPHASING RATE}

To model the observed decrease in the qubit dephasing rate with the MPIJIS applied pump power, we update the filtering parameter $A$ of the resonator-MPIJIS system to include the effect of power saturation of the MPIJIS due to pump depletion, i.e., $A \rightarrow A(L)$.

Starting from the on-resonance relation [Eq. (D20)], we obtain the following approximation:

$$
L=L_{0}+\sqrt{L_{0}} \frac{\bar{n}_{n}^{\text {in }}\left(L_{0}\right)}{2 \bar{n}_{c}^{\text {in }}} .
$$

Extending it to frequencies within the device bandwidth gives

$$
L(\omega)=L_{0}(\omega)+\sqrt{L_{0}(\omega)} \frac{\bar{n}_{n}^{\mathrm{in}}\left(L_{0}\right)}{2 \bar{n}_{c}^{\mathrm{in}}} .
$$

Multiplying Eq. (E2) by the filter response of the readout resonator in the frequency domain, i.e., $R(\omega)$, yields

$$
L(\omega) R(\omega)=L_{0}(\omega) R(\omega)+\sqrt{L_{0}(\omega)} R(\omega) \frac{\bar{n}_{n}^{\mathrm{in}}\left(L_{0}\right)}{2 \bar{n}_{c}^{\mathrm{in}}} .
$$

Integrating the combined response over the frequency range spanned by the cutoff angular frequencies $\omega_{c 1}$ and 
$\omega_{c 1}$ gives

$$
A(L)=A+\frac{\bar{n}_{n}^{\mathrm{in}}\left(L_{0}\right)}{2 \bar{n}_{c}^{\mathrm{in}}} \int_{\omega_{c 1}}^{\omega_{c 2}} \sqrt{L_{0}(\omega)} R(\omega) d \omega
$$

where $A(L) \equiv \int_{\omega_{c 1}}^{\omega_{c 2}} L(\omega) R(\omega) d \omega, A \equiv \int_{\omega_{c 1}}^{\omega_{c 2}} L_{0}(\omega) R(\omega) d \omega$, and $\bar{n}_{n}^{\text {in }}\left(L_{0}\right) \equiv \frac{1}{\pi} \int_{\omega_{c 1}}^{\omega_{c 2}} N_{a}^{\text {in }}(\omega)\left[1-L_{0}(\omega)\right] d \omega$.

Furthermore, since $N_{a}^{\text {in }}(\omega)$ is a slowly varying function in the relevant frequency range $\left[\omega_{c 1}, \omega_{c 2}\right]$, we introduce an effective noise photon flux (per unit time) $\gamma_{n} \bar{n}_{n}$ such that $\bar{n}_{n}^{\text {in }}\left(L_{0}\right)=\gamma_{n} \bar{n}_{n} T_{t}$, where $T_{t}=\int_{\omega_{c 1}}^{\omega_{c 2}}\left[1-L_{0}(\omega)\right] d \omega$.

This allows us to cast Eq. (E4) in a simpler form:

$$
A(L)=A+C \frac{T_{t}}{\rho^{2}} \int_{\omega_{c 1}}^{\omega_{c 2}} \sqrt{L_{0}(\omega)} R(\omega) d \omega,
$$

where $C=\left(2 \gamma_{n} / \pi \gamma_{c}\right) \rho_{n}^{2}$ and $\rho_{n}^{2} \equiv 4 g_{3}^{2} \bar{n}_{n} / \gamma_{a} \gamma_{b}$.

When calculating the fit $T_{\varphi, D}$ drawn in Figs. 7(e) and 7(h), we take $C=0.02$, which is within the range of our system parameters.

\section{APPENDIX F: JPC HAMILTONIAN}

The bare Hamiltonian of a nondegenerate three-wave mixing device, comprising three parallel $L C$ resonators coupled via a dispersive nonlinear medium, can be written as as $[38,64]$

$$
\begin{aligned}
H_{0}= & \frac{\Phi_{X}^{2}}{2 L_{a}}+\frac{\Phi_{Y}^{2}}{2 L_{b}}+\frac{\Phi_{Z}^{2}}{2 L_{c}}+\frac{Q_{X}^{2}}{2 C_{a}}+\frac{Q_{Y}^{2}}{2 C_{b}}+\frac{Q_{Z}^{2}}{2 C_{c}} \\
& +K \Phi_{X} \Phi_{Y} \Phi_{Z},
\end{aligned}
$$

where $\Phi_{X}, \Phi_{Y}$, and $\Phi_{Z}$ are the generalized flux variables of the three resonators and $Q_{X}, Q_{Y}$, and $Q_{Z}$ are the corresponding charge variables. The first six terms of $H_{0}$ represent the energy of three independent harmonic oscillators $(\mathrm{a}, \mathrm{b}$, and $\mathrm{c})$ having angular frequencies $\omega_{a}=$ $1 / \sqrt{L_{a} C_{a}}, \omega_{b}=1 / \sqrt{L_{b} C_{b}}$, and $\omega_{c}=1 / \sqrt{L_{c} C_{c}}$ and characteristic impedances $Z_{a}=\sqrt{L_{a} / C_{a}}, Z_{b}=\sqrt{L_{b} / C_{b}}$, and $Z_{c}=\sqrt{L_{c} / C_{c}}$. Notably, the last term represents a trilinear mixing interaction between modes $\mathrm{X}, \mathrm{Y}$, and $\mathrm{Z}$ with coefficient $K$.

Here we assume that the angular frequencies of the three modes are distinct, the resonators are well in the underdamped regime, $\gamma_{a, b, c} \ll \omega_{a, b, c}$, where $\gamma_{a}, \gamma_{b}, \gamma_{c}$ are the corresponding photon escape rates, and $\gamma_{a}+\gamma_{b} \ll \omega_{b}-$ $\omega_{a}$, which supposes that the envelopes of the drive signals exciting these modes are slow compared with the respective drive frequencies.
Equivalently, the Hamiltonian $H_{0}$ can be expressed in terms of bosonic operators:

$$
\begin{aligned}
H_{0}= & \hbar \omega_{a} a^{\dagger} a+\hbar \omega_{b} b^{\dagger} b+\hbar \omega_{c} c^{\dagger} c \\
& +\hbar g_{3}\left(a^{\dagger}+a\right)\left(b^{\dagger}+b\right)\left(c^{\dagger}+c\right),
\end{aligned}
$$

where $a^{\dagger}, a, b, b^{\dagger}, c^{\dagger}$, and $c$ are the raising and annihilation operators associated with the three modes, which commute with each other and satisfy the standard commutation relations $\left[a, a^{\dagger}\right]=1,\left[b, b^{\dagger}\right]=1$, and $\left[c, c^{\dagger}\right]=1$. The coupling constant between the modes $g_{3}$ is assumed to be much smaller than the angular frequencies $\omega_{a}, \omega_{b}$, and $\omega_{c}$ and decay rates $\gamma_{a}, \gamma_{b}$, and $\gamma_{c}$.

By further working in the framework of the rotatingwave approximation and assuming a classical coherent drive (i.e., pump) at $\omega_{p}=\left|\omega_{b}-\omega_{a}\right|$ and $g_{3} \geq 0$, we obtain

$$
H_{3 \text { wave }}=\hbar\left|g_{a b}\right|\left(e^{i \phi_{p}} a b^{\dagger}+e^{-i \phi_{p}} a^{\dagger} b\right),
$$

where $g_{a b}=g_{3} \sqrt{\bar{n}_{c}} e^{-i \phi_{p}}$. In the derivation of Eq. (F3), we replaced the annihilation operator $c$ by its average value in the coherent state produced by the pump, where $\bar{n}_{c}$ is the average pump photon number and $\phi_{p}$ is the pump phase.

To find $g_{3}$, we express the mode amplitudes (i.e., $\Phi_{X}$, $\Phi_{Y}$, and $\left.\Phi_{Z}\right)$ in terms of the corresponding bosonic operators: $\Phi_{X}=\Phi_{X}^{0}\left(a+a^{\dagger}\right), \Phi_{Y}=\Phi_{Y}^{0}\left(b+b^{\dagger}\right)$, and $\Phi_{Z}=$ $\Phi_{Z}^{0}\left(c+c^{\dagger}\right)$, where $\Phi_{X}^{0}=\sqrt{\hbar Z_{a} / 2}, \Phi_{Y}^{0}=\sqrt{\hbar Z_{b} / 2}$, and $\Phi_{Z}^{0}=\sqrt{\hbar Z_{c} / 2}$ are the zero-point fluctuations of the generalized flux. Using these relations and Eqs. (F1) and (F2), we obtain the following link between $g_{3}$ and $K$ [38]:

$$
\hbar g_{3}=K \Phi_{X}^{0} \Phi_{Y}^{0} \Phi_{Z}^{0}
$$

In the case of the JPC, the mixing term $K \Phi_{X} \Phi_{Y} \Phi_{Z}$ originates from the JRM, which functions as a dispersive nonlinear mixing element. As seen in Fig. 12(a), the JRM consists of four nominally identical Josephson junctions with energy $E_{J}=\varphi_{0} I_{0}$ (i.e., a, b, c, and d) arranged in a Wheatstone bridge configuration, where $\varphi_{0}=\Phi_{0} / 2 \pi$ is the reduced flux quantum $\left(\Phi_{0}=h / 2 e\right)$ and $I_{0}$ is the critical current. The Josephson junctions of the JRM are shunted by linear inductors in the form of large inner Josephson junctions, each with inductance $L$. Externally applied magnetic flux threading the JRM, $\Phi_{\text {ext }}$, induces a direct current, $I_{\text {circ }}$, circulating in the outer loop.

For a symmetrical JRM, in which the areas of the four inner loops are equal, as shown in Fig. 12(b), the external flux threading them is $\Phi_{\text {ext }} / 4$, and for $0 \leq\left|\Phi_{\text {ext }}\right| \leq$ $1.4 \Phi_{0}$ (i.e., located on the primary flux lobe), no direct currents flow in the inner branches. It is straightforward to show that the JRM supports four spatial eigenmodes, three of which (X, Y, and Z) resonate at microwave frequencies [shown in Figs. 12(c)-12(e)] and a fourth (not 
Even parity $(p=0)$
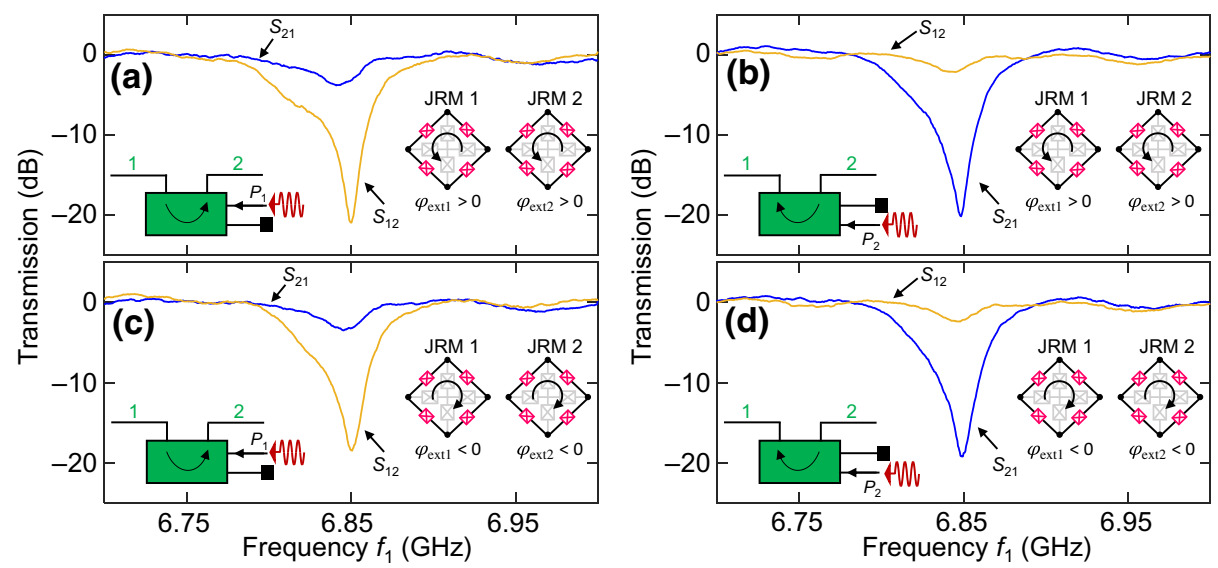

Odd parity $(p=1)$
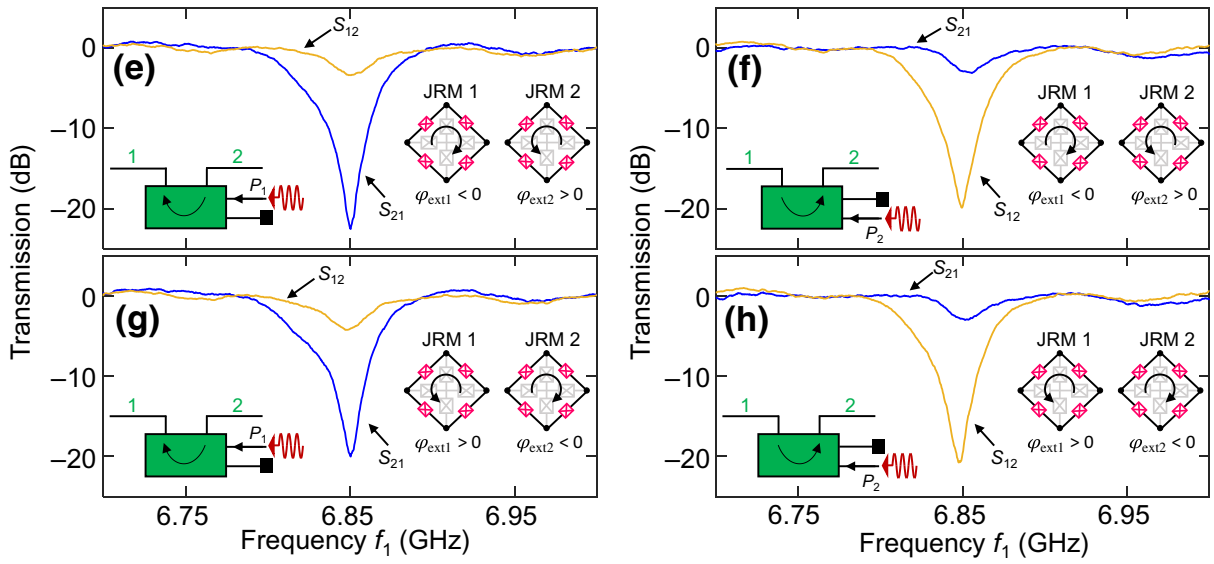

FIG. 13. Dependence of the transmission or isolation direction on the parity of the fluxes threading the two JRMs. Transmission parameters of the MPIJIS measured versus signal frequency. The measurement results shown on the left (right) are obtained with the pump driving $P_{1}\left(P_{2}\right)$ at $f_{p}=2.783 \mathrm{GHz}$. In (a)-(d), representing the even parity cases $(p=0)$, the magnetic fluxes threading the two JRMs are either $\varphi_{\text {ext1 }}>0$ and $\varphi_{\text {ext2 }}>0$ in (a),(b) or $\varphi_{\text {ext1 }}<0$ and $\varphi_{\text {ext2 }}<0$ in (c),(d). In (e)-(h), representing the odd parity cases $(p=1)$, the magnetic fluxes threading the two JRMs are either $\varphi_{\text {ext1 }}<0$ and $\varphi_{\text {ext2 }}>0$ in (e),(f) or $\varphi_{\text {ext1 }}>0$ and $\varphi_{\text {ext2 }}<0$ in (g),(h). The magnitude of the flux used is $\left|\varphi_{\text {ext } 1}\right|=\left|\varphi_{\text {ext } 2}\right|=2 \pi \times 1.12$ (i.e., $\Phi_{\text {ext }}=1.12 \Phi_{0}$ ), which corresponds to a magnetic field magnitude $\left|B_{\text {ext }}\right|=\left|\Phi_{\text {ext }}\right| / A_{\mathrm{JRM}} \cong 4 \times 10^{-6} \mathrm{~T}$ in the JRM plane, where $A_{\mathrm{JRM}}=(24 \mu \mathrm{m})^{2}$ is the area of the JRM loop.

shown) which is at dc [64]. As seen Figs. 12(c)-12(e), two of the eigenemodes, $\mathrm{X}$ and $\mathrm{Y}$, are differential, whereas $\mathrm{Z}$ is a common excitation. As inferred from Figs. 12(b)-12(e), the reduced branch fluxes representing these eigenmodes $\left(\varphi_{X}^{\prime} \equiv \Phi_{X}^{\prime} / \varphi_{0}, \varphi_{Y}^{\prime} \equiv \Phi_{Y}^{\prime} / \varphi_{0}\right.$, and $\left.\varphi_{Z}^{\prime} \equiv \Phi_{Z}^{\prime} / \varphi_{0}\right)$ can be expressed as orthogonal linear combinations of the reduced node fluxes of the JRM (i.e., $\left.\varphi_{i}=\Phi_{i} / \varphi_{0}, i=1,2,3,4\right): \varphi_{X}^{\prime}=\varphi_{1}-\varphi_{2}, \varphi_{Y}^{\prime}=\varphi_{3}-\varphi_{4}$, and $\varphi_{Z}^{\prime}=\left(\varphi_{1}+\varphi_{2}-\varphi_{3}-\varphi_{4}\right) / 2$. Since the branch fluxes across the JRM $\left(\Phi_{X}^{\prime}, \Phi_{Y}^{\prime}\right.$, and $\left.\Phi_{Z}^{\prime}\right)$ are only a fraction of the total generalized fluxes $\Phi_{X}, \Phi_{Y}$, and $\Phi_{Z}$ of the oscillators in Eq. (F1), they satisfy $\Phi_{X}^{\prime}=p_{a} \Phi_{X}, \Phi_{Y}^{\prime}=$ $p_{b} \Phi_{Y}$, and $\Phi_{Z}^{\prime}=p_{c} \Phi_{Z}$, where $p_{a} \approx L_{J 0} / L_{a}, p_{b} \approx L_{J 0} / L_{b}$, and $p_{c} \approx L_{J 0} / L_{c}$ are participation ratios representing the fraction of the spatial modes contained in the JRM and $L_{J 0}=\varphi_{0} / I_{0}$ is the linear inductance of the outer Josephson junctions.
For small $\varphi_{X}^{\prime}, \varphi_{Y}^{\prime}, \varphi_{Z}^{\prime} \ll 1$, the Hamiltonian of the shunted JRM reads $[42,64]$

$$
\begin{aligned}
H_{\mathrm{JRM}}= & -E_{J} \sin \left(\frac{\varphi_{\mathrm{ext}}}{4}\right) \varphi_{X}^{\prime} \varphi_{Y}^{\prime} \varphi_{Z}^{\prime} \\
& +\left[\frac{E_{L}}{2}+E_{J} \cos \left(\frac{\varphi_{\mathrm{ext}}}{4}\right)\right]\left(\frac{\varphi_{X}^{\prime 2}}{2}+\frac{\varphi_{Y}^{\prime 2}}{2}\right) \\
& +2\left[\frac{E_{L}}{4}+E_{J} \cos \left(\frac{\varphi_{\mathrm{ext}}}{4}\right)\right] \varphi_{Z}^{\prime 2} \\
& -4 E_{J} \cos \left(\frac{\varphi_{\mathrm{ext}}}{4}\right)
\end{aligned}
$$

where $\varphi_{\text {ext }}=\Phi_{\text {ext }} / \varphi_{0}$ is the reduced external flux and $E_{L}=$ $\varphi_{0}^{2} / L$ is the inductive energy of the large, inner Josephson junctions. The first term of $H_{\mathrm{JRM}}$ is a three-wave-mixing term, whereas the second and third terms are quadratic in 
the mode fluxes and therefore renormalize the mode frequencies. The fourth term is a constant (for a given external flux) that is independent of the mode fluxes.

To calculate $g_{3}$ we rewrite the mixing term of $H_{\text {JRM }}$ in the format of the right-hand side of Eq. (F4), which gives

$$
g_{3}=-\frac{E_{J}}{\hbar} \sin \left(\frac{\varphi_{\mathrm{ext}}}{4}\right) \frac{p_{a} p_{b} p_{c}}{\varphi_{0}^{3}} \Phi_{X}^{0} \Phi_{Y}^{0} \Phi_{Z}^{0} .
$$

Finally, substituting $\Phi_{X}^{0}=\sqrt{\hbar Z_{a} / 2}=\sqrt{\hbar \omega_{a} L_{a} / 2}, \Phi_{Y}^{0}=$ $\sqrt{\hbar Z_{b} / 2}=\sqrt{\hbar \omega_{b} L_{b} / 2}$, and $\Phi_{Z}^{0}=\sqrt{\hbar Z_{c} / 2}=\sqrt{\hbar \omega_{c} L_{c} / 2}$ in Eq. (F6) yields

$$
g_{3}=-\sin \left(\frac{\varphi_{\mathrm{ext}}}{4}\right) \sqrt{\frac{p_{a} p_{b} p_{c} \omega_{a} \omega_{b} \omega_{c}}{E_{J}^{\mathrm{eff}} / \hbar}},
$$

where $E_{J}^{\text {eff }}$ is the effectively available Josephson energy, which is proportional to $E_{J}$ with a numerical prefactor. This result shows that (1) the sign of the coupling constant $g_{3}$ is opposite that of the external flux $\varphi_{\text {ext }}$ in the range $-1.4 \times 2 \pi \leq \varphi_{\text {ext }} \leq 1.4 \times 2 \pi$ (corresponding to the JPCs being flux-biased on the primary flux lobe) and (2) the magnitude of $g_{3}$ varies with $\left|\sin \left(\frac{\varphi_{\text {ext }}}{4}\right)\right|$; for example, it vanishes (no mode coupling) when $\varphi_{\text {ext }}=0$. In Figs. 12(f)-12(h), we show illustrations of the direction of the direct current flowing in the JRM for $\varphi_{\text {ext }}<0, \varphi_{\text {ext }}=$ 0 , and $\varphi_{\text {ext }}>0$, respectively, which follows the relation $\operatorname{sgn} \varphi_{\text {ext }}=\operatorname{sgn} I_{\text {circ }}$. The black circles in Fig. 12(g) corresponding to $\varphi_{\text {ext }}=0$ indicate that the circulating current is zero.

On the basis of Eq. (F7), we can rewrite Eq. (F3) in the general form

$$
H_{3 \mathrm{wave}}=\hbar\left|g_{a b}\right|\left(e^{i \phi_{p}^{\prime}} a b^{\dagger}+e^{-i \phi_{p}^{\prime}} a^{\dagger} b\right),
$$

where $\phi_{p}^{\prime}=\phi_{p}+n_{g} \pi$ and

$$
n_{g}= \begin{cases}0, & \varphi_{\mathrm{ext}} \leq 0 \\ 1, & \varphi_{\mathrm{ext}}>0\end{cases}
$$

\section{APPENDIX G: DEPENDENCE OF THE MPIJIS RESPONSE ON APPLIED FLUX PARITY}

In Fig. 13, we experimentally demonstrate that the direction of the transmission or isolation of the MPIJIS device is determined not only by the sign of the phase difference of the pumps feeding the two JPCs but also by the orientation parity of the magnetic fields biasing the two JPCs or alternatively the parity of the circulating currents flowing in the JRMs, which, in turn, determines the sign of the coupling between the eignmodes of the JPCs. As seen in the measurement results in Fig. 13, for the same pump, feeding the same pump port $P_{1}$ or $P_{2}$, the direction of the transmission or isolation can be reversed by flipping the magnetic flux in one JRM loop or preserved by flipping the magnetic flux threading two loops. This result opens the door for magnetic field detection applications, such as the detection of the orientation parity of weak magnetic sources using simple microwave transmission measurements as outlined in Appendix K. It is important to point out here that although the parity of the magnetic fields biasing the JPCs plays a role in setting the directionality of the MPIJIS, it does not generate its nonreciprocal response, which is induced primarily by the pump phase difference. It is also important to outline here that such a systematic investigation of the effect of the parity of the applied fluxes on the MPIJIS directionality is made possible owing to the single-pump feature of the device. Without it, pinning down this effect is considerably more challenging.

In the experiment, we cannot determine the sign of $I_{\text {circ }}$ or $\varphi_{\text {ext }}$ flux-biasing the JPCs directly. We only know the sign of the direct current $I_{\text {coil }}$ generated by the roomtemperature current source, which biases the small superconducting coil attached to each JPC. Therefore, to determine the parity of $\varphi_{\text {ext1 }}$ and $\varphi_{\text {ext2, we take the following }}$ steps: (1) We make an arbitrary assumption regarding $\varphi_{\text {ext }}$ of one JPC; for example, $\operatorname{sgn} \varphi_{\text {ext1 }}=\operatorname{sgn} I_{\text {circl }}=\operatorname{sgn} I_{\text {coill }}$, where $I_{\text {coill }}$ is considered negative only if it is smaller than $I_{\text {offset } 1}$, which is the direct current for which the JPC is biased at the maximum frequency of the primary flux lobe (corresponding to $\varphi_{\text {ext1 }}=0$ ). In general, $I_{\text {offset }}$ is slightly off zero when there is a nonzero magnetic field within the Cryoperm magnetic shield can enclosing the device. (2) We flux-bias JPC 1 at $I_{\text {coill }}<I_{\text {offset } 1}$, for which we assume $\varphi_{\text {ext1 }}<0$. (3) We set $I_{\text {coil2 }}$ such that it is negative (i.e., $I_{\text {coil2 }}<I_{\text {offset2 }}$ ) and aligns the resonance frequency of JPC 2 with that of JPC 1 at $I_{\text {coill }}$. (4) We apply a pump tone to $P_{1}$ (for which $\varphi_{p}=-\pi / 2$ ) and tune its frequency and power to yield near-unity transmission in one direction and isolation in the other direction. (5) Since $\operatorname{sgn} g_{3}=$ $-\operatorname{sgn} \varphi_{\text {ext }}$, the sign of $g_{3}$ of both JPCs is the same if $\operatorname{sgn} \varphi_{\text {ext1 }}=\operatorname{sgn} \varphi_{\text {ext2 }}$. Thus, if we obtain in step $4\left|S_{21}\right| \rightarrow 1$ and $\left|S_{12}\right| \rightarrow 0$, this implies on the basis of Eqs. (A2) and (A3) that the sign of $g_{3}$ of both JPCs is the same (i.e., $p=0$ ), otherwise the response is reversed. (6) Once we find in step 5 a pair $\left(I_{\text {coill }}, I_{\text {coil2 }}\right)$ that gives rise to the same $g_{3}$ sign based on the device response, we can determine the other parity configurations in a self-consistent manner, including for a pump fed through $P_{2}$.

\section{APPENDIX H: CALIBRATION OF MPIJIS SCATTERING PARAMETERS}

The reference level for unity (or close to unity) transmission of the MPIJIS device (in the range from - 0.5 to $0 \mathrm{~dB}$ ) is determined by its off-resonance transmission response when the pump is off. This is because JPCs function as perfect mirrors when the pump is off, and 


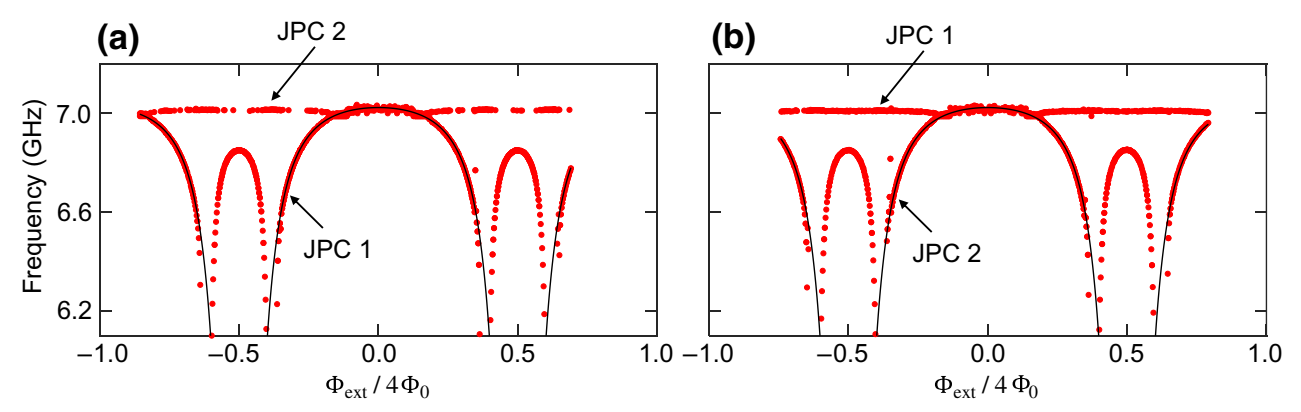

FIG. 14. JPC resonance frequency versus external magnetic flux. (a),(b) Resonance frequency of mode a of the two JPCs that form the MPIJIS as a function of the external magnetic flux threading the first and second JRMs, respectively. For the measurements shown in (a),(b), mode a of JPC 2 and JPC 1 is parked at its maximum frequency via a constant magnetic flux applied to JRM 2 and JRM 1, respectively. The solid black curves represent theory fits. The fits use the same device parameters in both plots.

the on-chip superconducting hybrids are lossless. More specifically, the off-resonance $0-\mathrm{dB}$ reference value, which we later subtract from the device transmission measurement versus frequency, is calculated as the average of about 100-300 transmission data points away from the resonance. This is done to dilute the effect of frequencydependent ripples in the transmission response when we are determining the $0-\mathrm{dB}$ reference value. These relatively low-frequency and small-amplitude ripples in the scattering parameter measurements, as seen in Figs. 2 and 13, are generally difficult to avoid and originate from reflections in the coaxial lines that connect the device under test and the circulator ports. Such a calibration method, based on the off-resonance, pump-off response, or a similar variation in which the pump-off response is subtracted from the pump-on curve, is commonly used in determining the amplification gain of JPAs and JPCs.

To calibrate the reflection parameters of the MPIJIS device, we rely on the fact that the two input lines that are coupled to the MPIJIS ports are nominally identical by design. That is, we set them to have the same attenuation and cable lengths both inside and outside the fridge down to the device ports. Furthermore, we verify that the transmission magnitudes versus frequency, measured through these lines at room temperature, agree to within less than 1 $\mathrm{dB}$.

Using this fact, we calibrate the reflection magnitude for the pump-off case by using the following useful relations. Let $I$ represent the attenuation of the input lines as a function of frequency in decibels (takes negative values) and let $O_{1}\left(O_{2}\right)$ represent the gain of output line 1 (2) as a function of frequency in decibels (take positive values). Let also $\left|S_{11}\right|\left(\left|S_{22}\right|\right)$ represent the calibrated reflection off port 1 (2) of the MPIJIS in decibels (takes negative values) and let $\left|S_{21}\right|\left(\left|S_{12}\right|\right)$ represent the calibrated transmission magnitude from port 1 to port 2 (port 2 to port 1 ) in decibels (takes nonpositive values). Then the reflection parameters, i.e., $\left|R_{11}\right|$ (decibels) and $\left|R_{22}\right|$ (decibels), as measured by the vector network analyzer are given by

$$
\begin{aligned}
& \left|R_{11}\right|=I+O_{1}+\left|S_{11}\right|, \\
& \left|R_{22}\right|=I+O_{2}+\left|S_{22}\right| .
\end{aligned}
$$

Similarly, the transmission parameters, i.e., $\left|T_{21}\right|$ (decibels) and $\left|T_{12}\right|$ (decibels), are given by

$$
\begin{aligned}
& \left|T_{21}\right|=I+O_{2}+\left|S_{21}\right|, \\
& \left|T_{12}\right|=I+O_{1}+\left|S_{12}\right| .
\end{aligned}
$$

Once $\left|S_{21}\right|$ is determined using the off-resonance method (described above), $\left|S_{22}\right|$ can be extracted from $\left|R_{22}\right|$ measurement. This is because the sum $I+\mathrm{O}_{2}$ is shared by both $\left|R_{22}\right|$ and $\left|T_{21}\right|$. The same applies to finding $\left|S_{11}\right|$ after $\left|S_{12}\right|$ is determined. This is because the sum $I+O_{1}$ is shared by $\left|T_{12}\right|$ and $\left|R_{11}\right|$ measurements.

\section{APPENDIX I: MPIJIS CHARACTERIZATION MEASUREMENTS}

In Fig. 14(a) we show the dependence of the resonance frequency of mode a of JPC 1 and JPC 2 on the applied external flux threading the respective JRM. In Fig. 14(a) [Fig. 14(b)], the resonance frequency of mode a of JPC 1 (JPC 2) is varied as a function of the external flux applied to JRM 1 (JRM 2), while the resonance frequency of mode a of JPC 2 (JPC 1) is parked at its maximum [corresponding to a fixed applied flux threading JRM 2 (JRM 1). The filled red circles represent measured data points, while the solid black curves represent theory fits to the major flux lobes of the JPCs. The theoretical model used to produce the fits is the same as in Refs. [39,42]. It is worthwhile noting that both fits use the same device 
(a)

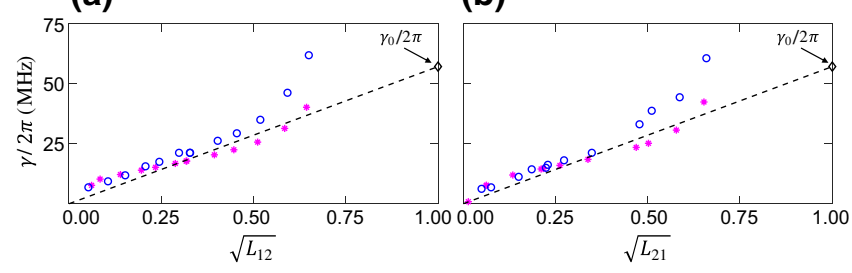

FIG. 15. Dynamical bandwidth of the MPIJIS $(\gamma / 2 \pi)$ versus its amplitude attenuation $\sqrt{L_{12}}=\left|S_{12}\right|$ and $\sqrt{L_{21}}=\left|S_{21}\right|$ depicted in (a),(b), respectively. The blue circles represent data extracted from the measurements shown in Fig. 3 for different pump drives fed to $P_{1}$ and $P_{2}$. The magenta stars are theoretical results extracted from the calculated curves. The black diamond corresponds the effective linear bandwidth of the MPIJIS when it is off $(L=1)$. The dashed black line corresponds to the relation $\gamma=\gamma_{0} \sqrt{L}$.

parameters. This suggests that fabrication variations on the same chip are small and that the JPCs are indeed nominally identical. From the fits, we extract the following device parameters: $I_{0}=2.82 \mu \mathrm{A}$, the critical current of the outer Josephson junctions of the JRM; $f_{\max }=7.0232 \mathrm{GHz}$, the maximum resonance frequency of mode a; $Z_{\text {res }}=51.1 \Omega$, the characteristic impedance of the JPC resonator; $L_{J 0} / L=$ 3.1 , the Josephson inductance ratio between the outer and inner JRM junctions; and $L_{J 0} / L_{s}=5$, the inductance ratio between the outer JRM junctions and the parasitic series inductance of the superconducting wire in each arm.

The fact that the measured maximum frequencies of the JPCs of the MPIJIS and MPIJDA devices integrated into the QRMCM (all taken from the same wafer) fall within a window of less than $40 \mathrm{MHz}$ (less than $10 \mathrm{MHz}$ on the same chip) indicates that the JRM inductance is quite uniform across the wafer and that it should be quite feasible, in the future, to operate multiple broadband versions of the MPIJIS and MPIJDA devices using a single microwave pump to further reduce the number of microwave generators required per board or multiple boards.

To investigate the dependence of the dynamical bandwidth of the MPIJIS, $\gamma / 2 \pi$, on the transmission dip in the isolated direction, $L_{i j}=\min \left|S_{i j}\right|^{2}$, we plot in Fig. 15 using blue circles the extracted bandwidths corresponding to the frequency difference of the $3-\mathrm{dB}$ points above the minimum of the measured curves (some of which are presented in Fig. 3). We also plot using magenta stars the calculated bandwidths extracted from the theoretical curves. As seen in Figs. 15(a) and 15(b), in which $\gamma / 2 \pi$ is plotted as a function of $\sqrt{L_{12}}$ and $\sqrt{L_{21}}$, respectively, corresponding to the MPIJIS operated in the forward $1 \rightarrow 2$ and backward $2 \rightarrow 1$ directions, the calculated bandwidths exhibit good agreement with the measured data, especially in the limit of vanishing $L_{i j}$, which corresponds to large isolations. This behavior mimics the shrinking bandwidths of Josephson amplifiers, such as JPAs and JPCs, in the limit of large gains due to the bandwidth-amplitude gain product, which is characteristic of resonant-structure-based parametric devices $[38,40]$. To highlight this observed behavior in the MPIJIS case, we plot using a black dashed line the relation $\gamma=\gamma_{0} \sqrt{L}$, where $\gamma_{0}=2 \gamma_{a} \gamma_{b} /\left(\gamma_{a}+\gamma_{b}\right)$, indicated by a black diamond, is the effective linear bandwidth of the JPC without a pump, $\gamma_{0} / 2 \pi=57 \mathrm{MHz}$, where $\gamma_{a} / 2 \pi=40 \mathrm{MHz}$ and $\gamma_{b} / 2 \pi=100 \mathrm{MHz}$.

Another related figure of merit is the tunable bandwidth of the device. In Fig. 16, we show a tunable bandwidth measurement of the MPIJIS. In this measurement, we vary the magnetic fluxes threading the two JRMs in tandem and adjust the pump power and frequency to yield isolation of more than $18 \mathrm{~dB}$ in the attenuated signal direction $\left|S_{12}\right|^{2}$ (orange curves) or $\left|S_{21}\right|^{2}$ (blue curves) depending on which pump port is driven, i.e., $P_{1}$ in Fig. 16(a) and $P_{2}$ in Fig. 16(b). As seen from this measurement, the MPIJIS possesses a tunable bandwidth of about $300 \mathrm{MHz}$ in both operation modes. This bandwidth is determined and limited by the tunable bandwidths of the two JPCs, the resonance frequency matching of modes $\mathrm{a}$ and $\mathrm{b}$ as a function of the applied flux, and the bandwidths of the on-chip $90^{\circ}$ hybrids.

Lastly, we show in Fig. 17 the saturation power of the MPIJIS in the forward direction [Fig. 17(a)] and the backward direction [Fig. 17(b)] for the working point presented in Fig. 2. This figure of merit represents the maximum input signal power that the device can handle for a fixed pump frequency $f_{p}=2.758 \mathrm{GHz}$ and pump power before its transmission or isolation response increases or decreases by $1 \mathrm{~dB}$. As seen in Fig. 17, the saturation power of the MPIJIS, indicated by the red circles, is about $-121 \mathrm{dBm}$ and it is determined by a change in

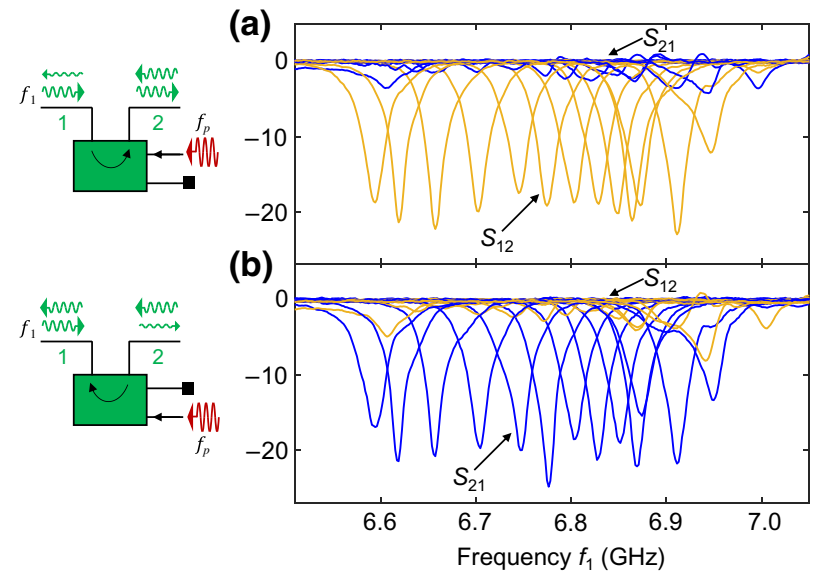

FIG. 16. Tunable bandwidth measurement. The resonance frequencies of the JPCs are varied in tandem by our varying the magnetic fluxes threading the two JRMs. For each flux working point, the pump frequency and power are adjusted to yield isolation of more than $18 \mathrm{~dB}$ in the relevant signal direction depending on which pump port is driven, i.e., $P_{1}$ in (a) and $P_{2}$ in (b). 
the isolation magnitude, which, notably, occurs at about $14 \mathrm{~dB}$ lower than the onset of the observed change in the transmission magnitude. While this measured saturation power is smaller than the saturation power reported in the proof-of-principle PCB-integrated MPIJIS [35] by about $12 \mathrm{~dB}$, it falls within the measured saturation power range of JPCs operated in amplification or frequencyconversion mode [38,43]. Since the power-handling capacity of JPCs is the limiting factor in this case, we expect this figure of merit of the MPIJIS to significantly improve as high-saturation-power JPCs become available in the future.

\section{APPENDIX J: QRMCM EXPERIMENTAL SETUP}

A detailed diagram of the setup used for measuring the qubit-QRMCM system is shown in Fig. 18. The main results obtained with this setup are shown in Figs. 5-7.

As seen in Fig. 18, we mount the qubit chip and the QRMCM in two separate Cryoperm magnetic shield cans. With the qubit shield covered with an Eccosorb lid. The setup includes five main sets of lines inside the fridge: (1) A qubit input line, colored yellow, that carries qubit pulses and that couples to the qubit via a feedline on the chip. (2) Input and output lines for readout, colored red, that carry input readout signals through the QRMCM toward the readout resonator and carry the reflected readout signals back through the QRMCM toward the output line, which includes a commercial wideband directional coupler
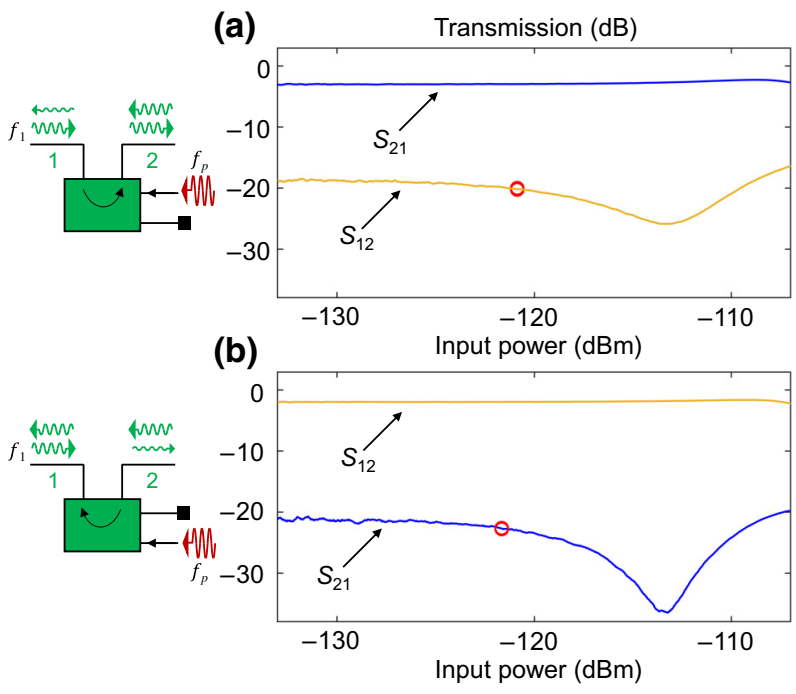

FIG. 17. Saturation power measurement of the MPIJIS. (a),(b) Transmission parameters of the MPIJIS, i.e., $\left|S_{21}\right|^{2}$ (blue) and $\left|S_{12}\right|^{2}$ (orange), measured as a function of the signal input power for pump drives applied to port $P_{1}$ and port $P_{2}$, respectively,. The red circles indicate the maximum input power for which the transmission magnitude of the MPIJIS changes by $1 \mathrm{~dB}$ relative to the low-power value. that allows measurement of the QRMCM in the opposite direction, an off-the-shelf low-pass filter with a cutoff at $8 \mathrm{GHz}$ at the mixing chamber, and a $\mathrm{Nb}$-Ti superconducting coaxial line that connects the filter to a standard Caltech low-noise HEMT amplifier (SN850D) mounted at the 4-K stage (specified to have a noise temperature of about $3-5 \mathrm{~K}$ in the range from 4 to $11 \mathrm{GHz}$ when measured at $18.6 \mathrm{~K})$. Following the HEMT, the readout signals are filtered, amplified, downconverted, and digitized at the room-temperature stage with use of standard electronic equipment. (3) Input and output lines, colored magenta, that are used for setting the working points of the MPIJIS and MPIJDA devices and measuring the QRMCM in the forward and backward directions. (4) Input and return lines, colored black, for feeding the pump drives to the MPIJIS and MPIJDA devices. These input lines include a $20-\mathrm{dB}$ attenuator at the 4-K stage and two identical commercial filters, located at the still and the mixing chamber stages, and a commercial wideband directional coupler for attenuating the pump tones by routing unused portions through the return lines, where they are dissipated at 50$\Omega$ terminations at the $4-\mathrm{K}$ stage. To enable the operation of the reconfigurable directional device as an amplifier (MPIJDA) or as an isolator (MPIJIS), we connect it to two sets of pump lines that have different filters incorporated into them. Depending on the desired mode of operation, we switch between pump lines that include low-pass filters with a $5.5-\mathrm{GHz}$ cutoff that allow the passage of lowfrequency pump tones in the MPIJIS case and bandpass filters that allow the transmission of high-frequency pump tones in the $11-18-\mathrm{GHz}$ band suitable for the MPIJDA case. The pump lines connected to the single-pump MPIJIS device are hardwired with low-pass filters only (the same kind as used for the reconfigurable device). Since the reconfigurable directional device requires the feeding of two pump signals into its two pump ports, we use a $90^{\circ}$ hybrid to evenly split the power of a single microwave generator (in each operation mode). To set the required phase shift between the two pump signals and compensate for any asymmetry in the attenuation of the lines, we incorporate a tunable attenuator and a phase shifter into one of the pump lines at the room-temperature stage. (5) Direct current lines (not shown) used for flux-biasing the small superconducting coils attached to the directional Josephson devices. These lines consist of two parts, resistive dc lines from the room-temperature stage to the 4-K stage followed by superconducting de lines from the 4-K stage to the mixing chamber.

Figure 19 shows a broadband transmission measurement result for the QRMCM experiment setup obtained with a vector network analyzer. The measurement includes the attenuation of the input line, the transmission of the QRMCM, and the amplification of the output line. The main features seen in this measurement result is set by the Purcell filter incorporated into the QRMCM; namely, 


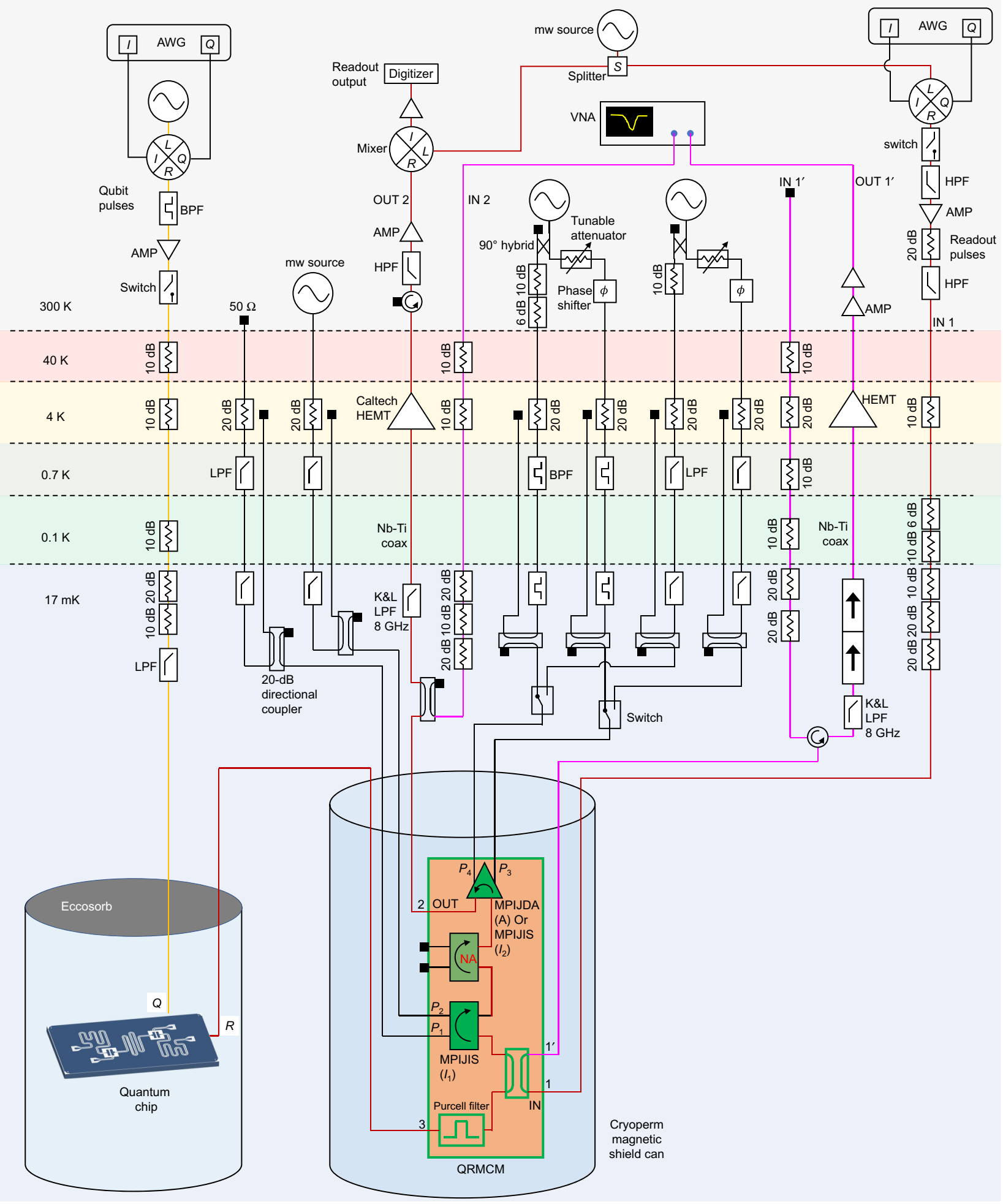

FIG. 18. The experimental setup used to obtain the measurement results shown in Figs. 5-7. Input and output lines colored red carry readout signals. The input line colored yellow carries qubit pulses. Input and output lines colored magenta are used to measure the QRMCM in the forward and backward directions. Input and return lines colored black carry pump drives. See the main text for details. AMP, amplifier; AWG, arbitrary waveform generator; BPF, bandpass filter; HPF, high-pass filter; mw, microwave; NA, not applicable; and VNA, vector network analyzer. 


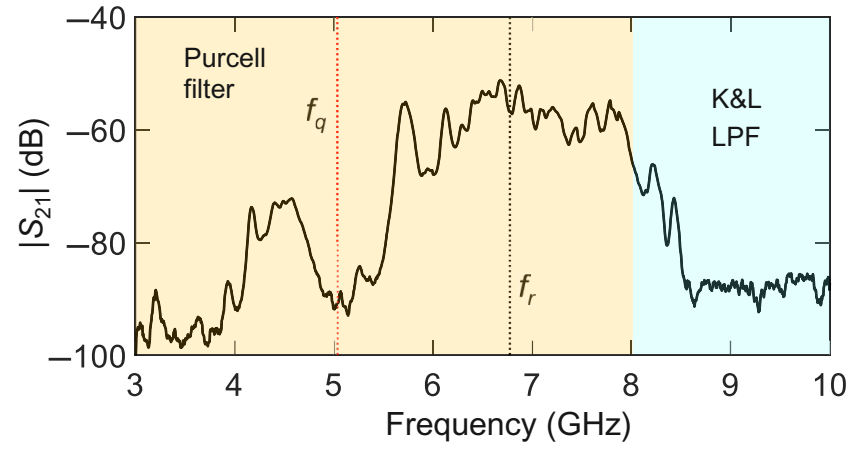

FIG. 19. Uncalibrated QRMCM transmission measurement. The transmission measurement is taken using a vector network analyzer in the frequency range from 3 to $10 \mathrm{GHz}$. It includes the attenuation of the input line, the transmission of the QRMCM, and the amplification of the output line. The vertical dashed red and black lines indicate the location of the qubit and readout frequencies, respectively. The shaded areas on the left and the right highlight the parts of the transmission landscape of the QRMCM that are dominated by the response of the Purcell filter and the off-the-shelf low-pass filter (LPF), respectively.

the transmission peak plateau seen surrounding the readout frequency and the strong attenuation encompassing the qubit frequency. The locations of these two frequencies are indicated by dashed vertical black lines.

Further details regarding the design, fabrication, packaging, and performance of the Purcell filter and the superconducting directional coupler can be found in Refs. [35,48].

\section{QRMCM versus magnetic isolator setup}

Figure 20 shows a detailed diagram of the setup used for comparing the performance of the QRMCM and two commercial broadband magnetic isolators in the output chain. In particular, this setup is used to obtain the data shown in Figs. 8(b), 8(c), and 9. In general, this setup is very similar to the one shown in Fig. 18. It differs from the previous setup mainly in the arrangement of the attenuation and filtering on some of the input lines and in the incorporation of two microwave switches and another wideband directional coupler, which enable us to measure the qubit using the QRMCM and the magnetic isolators in the same cooldown.

\section{Qubit measurement parameters}

The qubit used in the QRMCM experiment is a single Josephson junction transmon with anharmonicity of $330 \mathrm{MHz}$ capacitively coupled to a superconducting waveguide resonator measured in reflection. The qubit chip has a separate input port, through which we control the qubit, which is separate from the readout input line connected to the QRMCM. The qubit and readout frequencies are 5.033703 and $6.77698 \mathrm{GHz}$, respectively. The readout resonator bandwidth, $\kappa / 2 \pi$, is $1.1 \mathrm{MHz}$, while the qubit-state-dependent readout frequency shift, $\chi / 2 \pi$, is $0.5 \mathrm{MHz}$. The readout pulse duration and integration time, $T_{m}$, applied in the MPIJIS-MPIJDA, MPIJIS-MPIJIS, and variable isolation experiments is $0.75 \mu \mathrm{s}$, with an average photon number $\bar{n}_{m} \simeq 30$. The qubit data are averaged over 2000 iterations. The pump frequency applied to the MPIJIS device in the MPIJIS-MPIJDA, MPIJIS-MPIJIS, and variable isolation experiments is $2.712 \mathrm{GHz}$, while the pump frequency applied to the reconfigurable directional device in the first two experiments is 16.452 and $2.648 \mathrm{GHz}$, respectively. In the comparison experiment (against the magnetic isolators; see Fig. 8) the pump frequencies applied to the MPIJIS $1\left(I_{1}\right)$ and MPIJIS 2 $\left(I_{2}\right)$ are 2.654 and $2.68 \mathrm{GHz}$, respectively, and we set $T_{m}=1.5 \mu \mathrm{s}$.

\section{APPENDIX K: DETECTION SCHEME OF ORIENTATION PARITY OF WEAK MAGNETIC SOURCES}

As demonstrated in Fig. 13, the isolation direction of the MPIJIS is determined not only by the phase gradient between the pumps feeding the two JPCs but also by the parity of the magnetic fields flux-biasing their JRMs. Here we show how this interesting property could potentially be used to detect the orientation parity of weak magnetic sources via direct microwave transmission measurements. In Figs. 21(a) and 21(b), we show an exemplary detection setup that relies on this property. The setup acts as a two-path interferometer for coherent microwave signals at frequency $f_{1}$. It consists of input and output quadrature couplers (i.e., $90^{\circ}$ hybrids) whose inner ports are coupled through two paths. The first (top) path incorporates a gyrator formed by two JPCs coupled via their $b$ mode [66]. The second (bottom) path includes a lossless transmission line, whose electrical length is an integer multiple of the signal wavelength at $f_{1}$. The signal frequency $f_{1}$ used in the scheme lies within the bandwidth of mode a of the JPCs. The two JPCs are operated in frequency-conversion mode without photon gain, i.e., signals entering port a (b) are transmitted to port b (a) with frequency conversion, and are driven through a dedicated $90^{\circ}$ hybrid using a single pump drive, whose frequency is set to $f_{p}=f_{2}-f_{1}$, where $f_{2}$ lies within the bandwidth of mode b. Assuming, without loss of generality, that the pump driving the gyrator is fed through port $P_{1}$ of the hybrid $\left(\varphi_{p}=-\pi / 2\right)$, the microwave signals traversing the gyrator acquire a differential phase, i.e., $2 \pi k$ or $(2 k+1) \pi, k \in \mathbb{Z}$, depending on the propagation direction of the signals (i.e., from JPC 1 to JPC 2 or vice versa) and the direction parity of the magnetic fields flux-biasing the JPCs, as illustrated in Figs. 21(a) and 21(b). If we further restrict ourselves, for simplicity, to transmission measurements of signals entering the upper external port of the left $90^{\circ}$ hybrid and exiting via the 


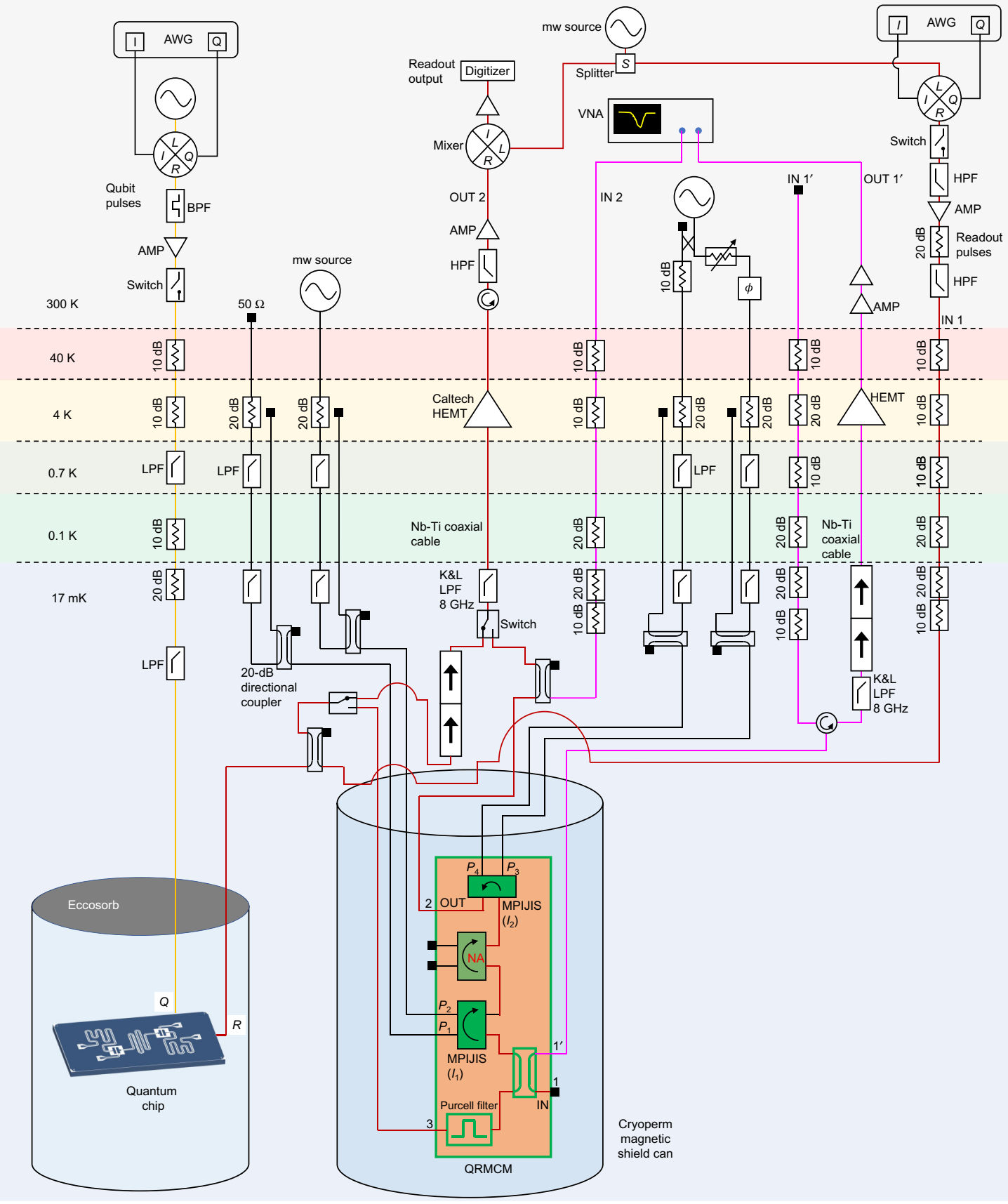

FIG. 20. The experimental setup used to obtain the measurement results shown in Figs. 8(b), 8(c), and 9. Input and output lines colored red carry readout signals. The input line colored yellow carries qubit pulses. Input and output lines colored magenta are used to measure the QRMCM in the forward and backward directions. Input and return lines colored black carry pump drives. See the main text for details. AMP, amplifier; AWG, arbitrary waveform generator; BPF, bandpass filter; HPF, high-pass filter; mw, microwave; NA, not applicable; and VNA, vector network analyzer.

upper external port of the right $90^{\circ}$ hybrid, we obtain zero transmission (no output signal, " 0 ") in the case of even parity of the biasing magnetic fields [Fig. 21(a)] and unity transmission (an output signal, "1") in the case of odd parity of the biasing magnetic fields [Fig. 21(b)].
In the former (latter) case, the zero (unity) transmission is the result of destructive (constructive) interference between waves propagating in the upper and bottom paths of the interferometer, which acquire opposite (equal) phases. 
It is important to point out that this proposed magnetic field parity detection scheme is not limited to two magnetic sources. It can be applied, in principle, to $N$ pairs of weak independent magnetic sources, as shown in Fig. 21(c), which flux-bias $N$ gyrators incorporated in series into the upper path of the interferometer. To prove the validity of this generalization, we apply the induction method. The first (base) step of the proof, in which we demonstrate the validity of the measurement setup for a given $N$, is shown in Figs. 21(a) and 21(b) for the case $N=1$. In the second
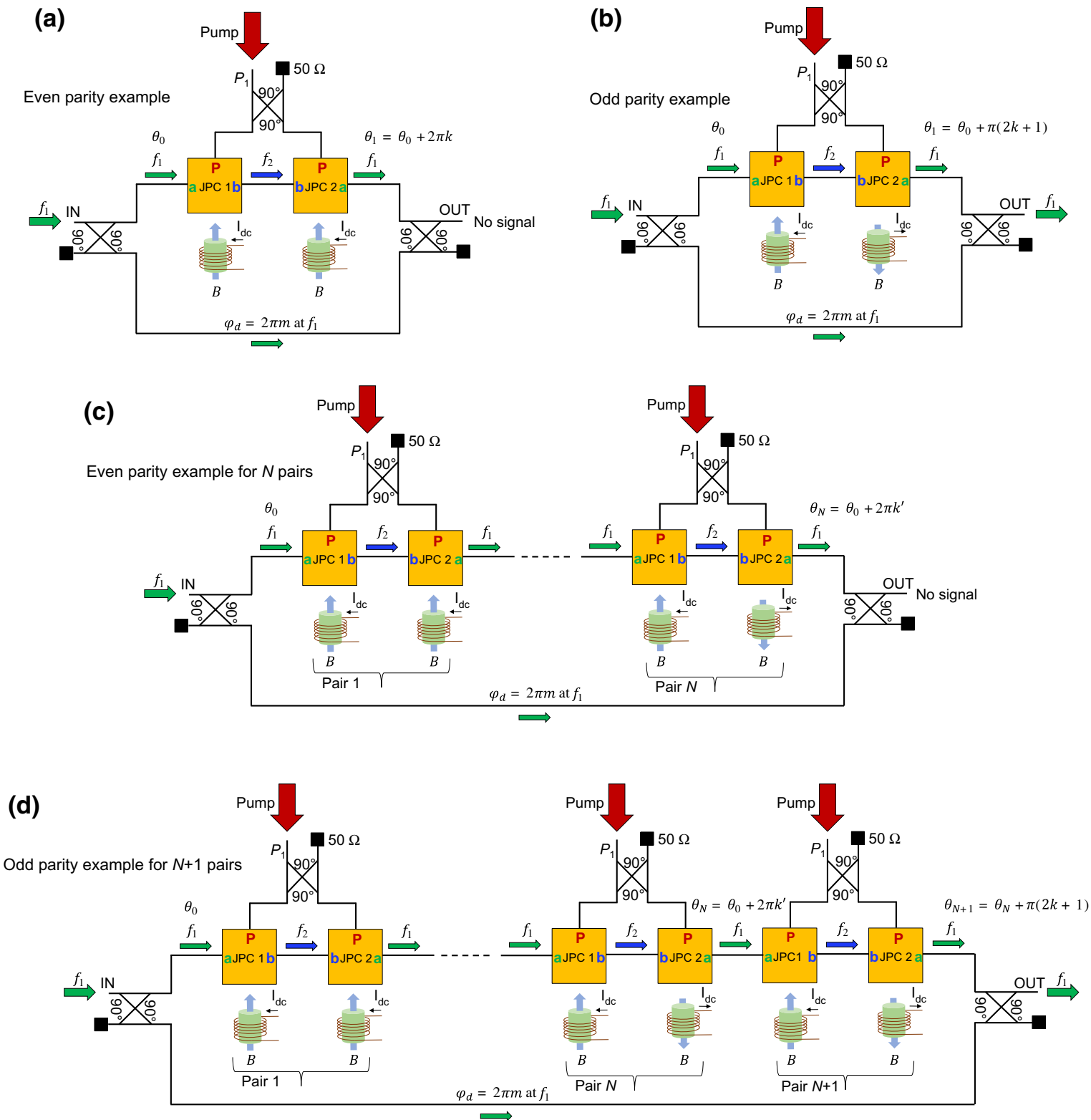

FIG. 21. Parity detection scheme for the orientation of weak magnetic sources. (a), (b) Interferometers formed by two $90^{\circ}$ hybrids, which interfere waves propagating in two paths, i.e., top and bottom. The top path includes a pair of JPCs coupled back-to-back via their $b$ mode. The two JPCs are operated in full frequency-conversion mode. They are driven by a single pump tone, which is fed through a third $90^{\circ}$ hybrid, which couples to the pump ports of the JPCs. The bottom path includes a lossless delay line, whose length is equal to an integer number of wavelengths at $f_{1}$. The scheme measures the transmission of a probe signal at $f_{1}$ input on the IN port of the left-hand-side hybrid and output at the OUT port of the right-hand-side hybrid. Minimum or maximum transmission though the device, resulting from respective destructive and constructive wave interference, is obtained if the orientation parity of the magnetic fluxes flux-biasing the JPCs is even (a) or odd (b). (c) A generalized detection scheme for $N$ pairs of magnetic sources, which flux-bias $N$ pairs of JPCs that are incorporated into the top path. The scheme response is illustrated for the case of even parity. (d) An extended version of the scheme in (c) with a total of $N+1$ pairs of JPCs and magnetic sources in the top path. In this case, the scheme response is illustrated for an odd parity measurement. 
(inductive) step, we demonstrate that the detection scheme is valid for $N+1$ pairs, given that it is valid for $N$, as partially illustrated in Fig. 21(d). To do that we consider four different cases:

(1) The first $N$ pairs of magnetic sources have an even number of aligned magnetic fields and the two magnetic fields biasing the $(N+1)$ th gyrator are aligned in the same direction (i.e., have even parity as well). Since the parity detection scheme works for the first $N$ pairs (resulting in destructive interference at the output), this means that the accumulated phase of the transmitted signal via the upper path is $\theta_{N}-\theta_{0}=2 \pi k^{\prime}$. By addition the $(N+1)$ th pair having even parity [similar to the case in Fig. 21(a)], the total accumulated phase at the output of the $(N+1)$ th pair is given by $\theta_{N+1}-\theta_{0}=2 \pi k^{\prime \prime}$, where $k^{\prime \prime}=k+k^{\prime}$ and $k, k^{\prime}, k^{\prime \prime} \in \mathbb{Z}$. Since the bottom path (including the diagonal lines of the hybrids) always yields an accumulated phase of $(2 m+1) \pi$, where $m \in \mathbb{Z}$, the waves propagating in the upper and bottom paths will destructively interefere at the output, thus confirming that the setup is valid for $N+1$ pairs in this case.

(2) The first $N$ pairs of magnetic sources have an even number of aligned magnetic fields and the two magnetic fields biasing the $(N+1)$ th gyrator are aligned in opposite directions (i.e., have odd parity), which means that the $N+1$ pairs have odd parity in total. By concatenation of the $(N+1)$ th pair having odd parity [similar to the case in Fig. 21(b)], the total accumulated phase at the output of the $(N+1)$ th pair is given by $\theta_{N+1}-\theta_{0}=\left(2 k^{\prime \prime}+1\right) \pi$. Consequently, the waves propagating along the upper and bottom paths will constructively interfere at the output (indicating odd parity of the whole chain), thus confirming that the setup is valid for $N+1$ pairs in this case as well.

(3) The first $N$ pairs of magnetic sources have an odd number of aligned magnetic fields and the two magnetic fields biasing the $(N+1)$ th gyrator are aligned in opposite directions (i.e., have odd parity). This means that the $N+1$ pairs have even parity in total. It is straightforward to show that the total accumulated phase at the output of the $(N+1)$ th pair is $\theta_{N+1}-\theta_{0}=2 \pi k^{\prime \prime}$, which yields destructive interference (no signal) at the device output, which is indicative of even parity.

(4) This is similar to case 3 except that the $(N+1)$ th gyrator is flux-biased with two aligned magnetic fields [i.e., having even parity as in Fig. 21(a)]. In this case, the whole chain $(N+1$ pairs $)$ will preserve the odd parity of the $N$ pairs. Since the total accumulated phase at the output of the $(N+1)$ th pair is given by $\theta_{N+1}-\theta_{0}=\left(2 k^{\prime \prime}+1\right) \pi$, the resultant wave interference at the output of the device is constructive, which, in turn, yields an output signal indicative of odd parity.

Such interferometric microwave detection schemes could potentially be used in a variety of applications. For example, they can be used to perform $Z$ operator parity checks on flux qubits or capacitively shunted flux qubits (CSFQs) using direct microwave transmission measurements [as opposed to (1) using ancilla qubits coupled to data qubits, (2) entangling them via single-qubit and two-qubit gates, and (3) measuring the final state of the ancilla qubits as done in the surface code architecture]. In such an application, each CSFQ is coupled to a JRM via a suitable mutual inductance. Since the qubit state is encoded in the direction of the persistent current in the CSFQ loop, opposite circulating currents will introduce opposite magnetic flux biases through the JRMs, which will determine the transmitted signal through the interferometric device based on the parity of the qubit states. As another example, they can be used to determine the strength and orientation of weak magnetic sources. This can be done by (1) coupling the source of the unknown magnetic field to one JRM of the interferometric gyrator device, (2) flux-biasing the other JRM using a controlled and well-characterized magnetic field, (3) injecting a pump tone into either $P_{1}$ or $P_{2}$, (4) applying a microwave signal to the device input, and (5) maximizing the transmitted microwave through the device by varying the power and frequency of the applied pump as well as the magnetic field of the controlled magnetic source. For a pump tone applied to $P_{1}$, for example, a maximum output signal is expected when the two magnetic fluxes threading the two JRMs have equal magnitudes but opposite signs [as seen in Fig. 21(b)].

We can estimate the range of magnetic fields $B_{\text {ext }}$ that are typically applied to JRM loops of area $A_{\text {JRM }}$ at the JRM plane by considering the bounds $0.1 \Phi_{0} / A_{\mathrm{JRM}}<\left|B_{\text {ext }}\right|<$ $\Phi_{0} / A_{\mathrm{JRM}}$. For a JRM loop area $A_{\mathrm{JRM}}$ of $100 \times 100 \mu \mathrm{m}^{2}$, the range is $2 \times 10^{-8} \mathrm{~T}<\left|B_{\text {ext }}\right|<2 \times 10^{-7} \mathrm{~T}$. Much smaller field bounds can be detected with use of a larger loop. However, bigger loops could add series linear inductance to the JRM, which is undesired (especially when it is comparable to the Josephson inductance of the Josephson junctions).

[1] C. L. Hogan, The ferromagnetic Faraday effect at microwave frequencies and its applications, Rev. Mod. Phys. 25, 253 (1953).

[2] C. Caloz, A. Alu, S. Tretyakov, D. Sounas, K. Achouri, and Z. L. Deck-Léger, Electromagnetic Nonreciprocity, Phys. Rev. Appl. 10, 47001 (2018). 
[3] L. Fan, J. Wang, L. T. Varghese, H. Shen, B. Niu, Y. Xuan, A. M. Weiner, and M. Qi, An all-silicon passive optical diode, Science 335, 447 (2012).

[4] G. Viola and D. P. DiVincenzo, Hall Effect Gyrators and Circulators, Phys. Rev. X 4, 021019 (2014).

[5] S. Bosco, F. Haupt, and D. P. DiVincenzo, Self-ImpedanceMatched Hall-Effect Gyrators and Circulators, Phys. Rev. Appl. 7, 024030 (2017).

[6] A. C. Mahoney, J. I. Colless, S. J. Pauka, J. M. Hornibrook, J. D. Watson, G. C. Gardner, M. J. Manfra, A. C. Doherty, and D. J. Reilly, On-Chip Microwave Quantum Hall Circulator, Phys. Rev. X 7, 011007 (2017).

[7] K. Fang, Z. Yu, and S. Fan, Photonic Aharonov-Bohm Eeffect Based on Dynamic Modulation, Phys. Rev. Lett. 108, 153901 (2012).

[8] K. Fang, Z. Yu, and S. Fan, Experimental demonstration of a photonic aharonov-bohm effect at radio frequencies, Phys. Rev. B 87, 060301(R) (2013).

[9] C. Galland, R. Ding, N. C. Harris, T. Baehr-Jones, and M. Hochberg, Broadband on-chip optical non-reciprocity using phase modulators, Opt. Express 21, 14500 (2013).

[10] J. Kerckhoff, K. Lalumière, B. J. Chapman, A. Blais, and K. W. Lehnert, On-Chip Superconducting Microwave Circulator from Synthetic Rotation, Phys. Rev. Appl. 4, 034002 (2015).

[11] L. Ranzani and J. Aumentado, Graph-based analysis of nonreciprocity in coupled-mode systems, New J. Phys. 17, 023024 (2015).

[12] A. Kamal, J. Clarke, and M. H. Devoret, Noiseless nonreciprocity in a parametric active device, Nat. Phys. 7, 311 (2011).

[13] M. H. Devoret and R. J. Schoelkopf, Superconducting circuits for quantum information: An outlook, Science 339, 1169 (2013).

[14] R. Vijay, D. H. Slichter, and I. Siddiqi, Observation of Quantum Jumps in a Superconducting Artificial Atom, Phys. Rev. Lett. 106, 110502 (2011).

[15] D. Ristè, J. G. Leeuwen, H.-S. Ku, K. W. Lehnert, and L. DiCarlo, Initialization by Measurement of a Superconducting Quantum bit Circuit, Phys. Rev. Lett. 109, 050507 (2012).

[16] P. Campagne-Ibarcq, E. Flurin, N. Roch, D. Darson, P. Morfin, M. Mirrahimi, M. H. Devoret, F. Mallet, and B. Huard, Persistent Control of a Superconducting Qubit by Stroboscopic Measurement Feedback, Phys. Rev. X 3, 021008 (2013).

[17] L. Sun, A. Petrenko, Z. Leghtas, B. Vlastakis, G. Kirchmair, K. M. Sliwa, A. Narla, M. Hatridge, S. Shankar, J. Blumoff, and L. Frunzio, Tracking photon jumps with repeated quantum non-demolition parity measurements, Nature 511, 444 (2014).

[18] N. Ofek, A. Petrenko, R. Heeres, P. Reinhold, Z. Leghtas, B. Vlastakis, Y. Liu, L. Frunzio, S. M. Girvin, L. Jiang, and M. Mirrahimi, Extending the lifetime of a quantum bit with error correction in superconducting circuits, Nature 536, 441 (2016).

[19] D. M. Pozar, Microwave Engineering (Wiley, Hoboken, 2005), 3rd ed.

[20] R. E. Collin, Foundations for Microwave Engineering (Wiley-Interscience, Hoboken, 2014), 2nd ed.
[21] K. Sliwa, M. Hatridge, A. Narla, S. Shankar, L. Frunzio, R. J. Schoelkopf, and M. H. Devoret, Reconfigurable Josephson Circulator/directional Amplifier, Phys. Rev. X 5, 041020 (2015).

[22] F. Lecocq, L. Ranzani, G. A. Peterson, K. Cicak, R. W. Simmonds, J. D. Teufel, and J. Aumentado, Nonreciprocal Microwave Signal Processing with a Field-Programmable Josephson Amplifier, Phys. Rev. Appl. 7, 024028 (2017).

[23] L. Ranzani, S. Kotler, A. J. Sirois, M. P. DeFeo, M. Castellanos-Beltran, K. Cicak, L. R. Vale, and J. Aumentado, Wideband Isolation by Frequency Conversion in a Josephson-Junction Transmission Line, Phys. Rev. Appl. 8, 054035 (2017).

[24] B. J. Chapman, E. I. Rosenthal, J. Kerckhoff, B. A. Moores, L. R. Vale, J. A. Mates, G. C. Hilton, K. Lalumiere, A. Blais, and K. W. Lehnert, Widely Tunable On-Chip Microwave Circulator for Superconducting Quantum Circuits, Phys. Rev. X 7, 041043 (2017).

[25] E. I. Rosenthal, B. J. Chapman, A. P. Higginbotham, J. Kerckhoff, and K. W. Lehnert, Breaking Lorentz Reciprocity with Frequency Conversion and Delay, Phys. Rev. Lett. 119, 147703 (2017).

[26] B. J. Chapman, E. I. Rosenthal, and K. W. Lehnert, Design of an On-Chip Superconducting Microwave Circulator with Octave Bandwidth, Phys. Rev. Appl. 11, 044048 (2019).

[27] N. R. Bernier, L. D. Toth, A. Koottandavida, M. A. Ioannou, D. Malz, A. Nunnenkamp, A. K. Feofanov, and T. J. Kippenberg, Nonreciprocal reconfigurable microwave optomechanical circuit, Nat. Commun. 8, 604 (2017).

[28] S. Barzanjeh, M. Wulf, M. Peruzzo, M. Kalaee, P. B. Dieterle, O. Painter, and J. M. Fink, Mechanical on-chip microwave circulator, Nat. Commun. 8, 953 (2017).

[29] A. Metelmann and A. A. Clerk, Nonreciprocal Photon Transmission and Amplification via Reservoir Engineering, Phys. Rev. X 5, 021025 (2015).

[30] C. Macklin, K. O’Brien, D. Hover, M. E. Schwartz, V. Bolkhovsky, X. Zhang, W. D. Oliver, and I. Siddiqi, A near-quantum-limited josephson traveling-wave parametric amplifier, Science 350, 307 (2015).

[31] A. Opremcak, C. H. Liu, C. Wilen, K. Okubo, B. G. Christensen, D. Sank, T. C. White, A. Vainsencher, M. Giustina, A. Megrant, and B. Burkett, High-Fidelity Measurement of a Superconducting Qubit Using an On-Chip Microwave Photon Counter, Phys. Rev. X 11, 011027 (2021).

[32] F. Lecocq, L. Ranzani, G. A. Peterson, K. Cicak, X. Y. Jin, R. W. Simmonds, J. D. Teufel, and J. Aumentado, Efficient Qubit Measurement with a Nonreciprocal Microwave Amplifier, Phys. Rev. Lett. 126, 020502 (2021).

[33] E. I. Rosenthal, C. M. Schneider, M. Malnou, Z. Zhao, F. Leditzky, B. J. Chapman, W. Wustmann, X. Ma, D. A. Palken, M. F. Zanner, and L. R. Vale, Efficient and LowBackaction Quantum Measurement Using a Chip-Scale Detector, Phys. Rev. Lett. 126, 090503 (2021).

[34] T. Thorbeck, S. Zhu, E. Leonard, Jr. R. Barends, J. Kelly, J. M. Martinis, and R. McDermott, Reverse Isolation and Backaction of the SLUG Microwave Amplifier, Phys. Rev. Appl. 8, 054007 (2017). 
[35] B. Abdo, N. T. Bronn, O. Jinka, S. Olivadese, A. D. Córcoles, V. P. Adiga, M. Brink, R. E. Lake, X. Wu, D. P. Pappas, and J. M. Chow, Active protection of a superconducting qubit with an interferometric josephson isolator, Nat. Commun. 10, 3154 (2019).

[36] B. Abdo, N. T. Bronn, O. Jinka, S. Olivadese, M. Brink, and J. M. Chow, Multi-path interferometric josephson directional amplifier for qubit readout, Quantum Sci. Technol. 3, 024003 (2018).

[37] B. Abdo, K. Sliwa, S. Shankar, M. Hatridge, L. Frunzio, R. Schoelkopf, and M. Devoret, Josephson Directional Amplifier for Quantum Measurement of Superconducting Circuits, Phys. Rev. Lett. 112, 167701 (2014).

[38] B. Abdo, A. Kamal, and M. H. Devoret, Nondegenerate three-wave mixing with the josephson ring modulator, Phys. Rev. B 87, 014508 (2013).

[39] B. Abdo, J. M. Chavez-Garcia, M. Brink, G. Keefe, and J. M. Chow, Time-multiplexed amplification in a hybrid-less and coil-less josephson parametric converter, Appl. Phys. Lett. 110, 082601 (2017).

[40] B. Abdo, F. Schackert, M. Hatridge, C. Rigetti, and M. H. Devoret, Josephson amplifier for qubit readout, Appl. Phys. Lett. 99, 162506 (2011).

[41] N. Bergeal, F. Schackert, M. Metcalfe, R. Vijay, V. E. Manucharyan, L. Frunzio, D. E. Prober, R. J. Schoelkopf, S. M. Girvin, and M. H. Devoret, Phase-preserving amplification near the quantum limit with a josephson ring modulator, Nature 465, 64 (2010).

[42] N. Roch, E. Flurin, F. Nguyen, P. Morfin, P. CampagneIbarcq, M. H. Devoret, and B. Huard, Widely Tunable, Nondegenerate Three-Wave Mixing Microwave Device Operating near the Quantum Limit, Phys. Rev. Lett. 108, 147701 (2012).

[43] B. Abdo, K. Sliwa, F. Schackert, N. Bergeal, M. Hatridge, L. Frunzio, A. D. Stone, and M. Devoret, Full Coherent Frequency Conversion between two Propagating Microwave Modes, Phys. Rev. Lett. 110, 173902 (2013).

[44] E. Flurin, N. Roch, J. D. Pillet, F. Mallet, and B. Huard, Superconducting Quantum Node for Entanglement and Storage of Microwave Radiation, Phys. Rev. Lett. 114, 090503 (2015).

[45] C. M. Caves, Quantum limits on noise in linear amplifiers, Phys. Rev. D 26, 1817 (1982).

[46] A. A. Clerk, M. H. Devoret, S. M. Girvin, F. Marquardt, and R. J. Schoelkopf, Introduction to quantum noise, measurement, and amplification, Rev. Mod. Phys. 82, 1155 (2010).

[47] X. Zhou, V. Schmitt, P. Bertet, D. Vion, W. Wustmann, V. Shumeiko, and D. Estève, High-gain weakly nonlinear fluxmodulated josephson parametric amplifier using a SQUID array, Phys. Rev. B 89, 214517 (2014).

[48] N. T. Bronn, Y. Liu, J. B. Hertzberg, A. D. Córcoles, A. A. Houck, J. M. Gambetta, and J. M. Chow, Broadband filters for abatement of spontaneous emission in circuit quantum electrodynamics, Appl. Phys. Lett. 107, 172601 (2015).

[49] A. A. Clerk and D. W. Utami, Using a qubit to measure photon-number statistics of a driven thermal oscillator, Phys. Rev. A 75, 042302 (2007).
[50] C. Rigetti, J. M. Gambetta, S. Poletto, B. L. Plourde, J. M. Chow, A. D. Córcoles, J. A. Smolin, S. T. Merkel, J. R. Rozen, G. A. Keefe, and M. B. Rothwell, Superconducting qubit in a waveguide cavity with a coherence time approaching $0.1 \mathrm{~ms}$, Phys. Rev. B 86, 100506(R) (2012).

[51] A. P. Sears, A. Petrenko, G. Catelani, L. Sun, H. Paik, G. Kirchmair, L. Frunzio, L. I. Glazman, S. M. Girvin, and R. J. Schoelkopf, Photon shot noise dephasing in the strong-dispersive limit of circuit QED, Phys. Rev. B 86, 180504(R) (2012).

[52] Z. Wang, S. Shankar, Z. K. Minev, P. Campagne-Ibarcq, A. Narla, and M. H. Devoret, Cavity Attenuators for Superconducting Qubits, Phys. Rev. Appl. 11, 014031 (2019).

[53] F. Yan, S. Gustavsson, A. Kamal, J. Birenbaum, A. P. Sears, D. Hover, T. J. Gudmundsen, D. Rosenberg, G. Samach, S. Weber, and J. L. Yoder, The flux qubit revisited to enhance coherence and reproducibility, Nat. Commun. 7, 12964 (2016).

[54] J.-D. Pillet, E. Flurin, F. Mallet, and B. Huard, A compact design for the josephson mixer: The lumped element circuit, Appl. Phys. Lett. 106, 222603 (2015).

[55] L.-H. Lu, S. Mohammadi, G. E. Ponchak, P. Bhattacharya, and L. P. Katehi, Design and implementation of micromachined lumped quadrature $\left(90^{\circ}\right)$ hybrids, Microwave Symp. Dig., IEEE MTT-S Int. 2, 1285 (2001).

[56] T. Roy, S. Kundu, M. Chand, A. M. Vadiraj, A. Ranadive, N. Nehra, M. P. Patankar, J. Aumentado, A. A. Clerk, and R. Vijay, Broadband parametric amplification with impedance engineering: Beyond the gainbandwidth product, Appl. Phys. Lett. 107, 262601 (2015).

[57] J. Mutus, T. C. White, R. Barends, Y. Chen, Z. Chen, B. Chiaro, A. Dunsworth, E. Jeffrey, J. Kelly, A. Megrant, and C. Neill, Strong environmental coupling in a josephson parametric amplifier, Appl. Phys. Lett. 104, 263513 (2014).

[58] G. Liu, T. C. Chien, X. Cao, O. Lanes, E. Alpern, D. Pekker, and M. Hatridge, Josephson parametric converter saturation and higher order effects, Appl. Phys. Lett. 111, 202603 (2017).

[59] C. Liu, T.-C. Chien, M. Hatridge, and D. Pekker, Optimizing josephson-ring-modulator-based josephson parametric amplifiers via full Hamiltonian control, Phys. Rev. A 101, 042323 (2020).

[60] L. Planat, R. Dassonneville, J. P. Martínez, F. Foroughi, O. Buisson, W. Hasch-Guichard, C. Naud, R. Vijay, K. Murch, and N. Roch, Understanding the Saturation Power of Josephson Parametric Amplifiers Made from SQUID Arrays, Phys. Rev. Appl. 11, 034014 (2019).

[61] Y.-F. Chen, D. Hover, S. Sendelbach, L. Maurer, S. T. Merkel, E. J. Pritchett, F. K. Wilhelm, and R. McDermott, Microwave Photon Counter Based on Josephson Junctions, Phys. Rev. Lett. 107, 217401 (2011).

[62] L. C. G. Govia, E. J. Pritchett, C. Xu, B. L. Plourde, M. G. Vavilov, F. K. Wilhelm, and R. McDermott, High-fidelity 
qubit measurement with a microwave-photon counter, Phys. Rev. A 90, 062307 (2014).

[63] R. Dassonneville, R. Assouly, T. Peronnin, P. Rouchon, and B. Huard, Number-Resolved Photocounter for Propagating Microwave Mode, Phys. Rev. Appl. 14, 044022 (2020).

[64] F. D. O. Schackert, A Practical Quantum-Limited Parametric Amplifier Based on the Josephson Ring Modulator. $\mathrm{Ph} . \mathrm{D}$. thesis (Yale University, 2013).
[65] H. S. Ku, F. Mallet, L. R. Vale, K. D. Irwin, S. E. Russek, G. C. Hilton, and K. W. Lehnert, Design and testing of superconducting microwave passive components for quantum information processing, IEEE Trans. Appl. Supercond. 21, 452 (2010).

[66] B. Abdo, M. Brink, and J. M. Chow, Gyrator Operation Using Josephson Mixers, Phys. Rev. Appl. 8, 034009 (2017). 